\title{
Spinal aging
}

Citation for published version (APA):

Jacobs, E. (2019). Spinal aging. [Doctoral Thesis, Maastricht University]. Maastricht University. https://doi.org/10.26481/dis.20191101ej

Document status and date:

Published: 01/01/2019

DOI:

10.26481/dis.20191101ej

Document Version:

Publisher's PDF, also known as Version of record

\section{Please check the document version of this publication:}

- A submitted manuscript is the version of the article upon submission and before peer-review. There can be important differences between the submitted version and the official published version of record.

People interested in the research are advised to contact the author for the final version of the publication, or visit the DOI to the publisher's website.

- The final author version and the galley proof are versions of the publication after peer review.

- The final published version features the final layout of the paper including the volume, issue and page numbers.

Link to publication

\footnotetext{
General rights rights.

- You may freely distribute the URL identifying the publication in the public portal. please follow below link for the End User Agreement:

www.umlib.nl/taverne-license

Take down policy

If you believe that this document breaches copyright please contact us at:

repository@maastrichtuniversity.nl

providing details and we will investigate your claim.
}

Copyright and moral rights for the publications made accessible in the public portal are retained by the authors and/or other copyright owners and it is a condition of accessing publications that users recognise and abide by the legal requirements associated with these

- Users may download and print one copy of any publication from the public portal for the purpose of private study or research.

- You may not further distribute the material or use it for any profit-making activity or commercial gain

If the publication is distributed under the terms of Article $25 \mathrm{fa}$ of the Dutch Copyright Act, indicated by the "Taverne" license above, 




\section{Spinal Aging}

Eva Jacobs 
Author: Eva Jacobs

ISBN: 978-94-6361-296-8

Cover design: Tania Watson (taniamwatson@gmail.com)

Layout: Erwin Timmerman, Optima Grafische Communicatie

Printed by: Optima Grafische Communicatie, Rotterdam

Financial support for the publication of this thesis was provided by:

Maastricht University (UM, Caphri), Maastricht University Medical Center

(MUMC+), Nederlands Orthopaedische Vereniging (NOV), Dutch Spine Society (DSS), Werkmeister GmbH, Motek Medical BV, Maxima Medisch Centrum Veldhoven, DSM Biomedical

\section{Copyright () E. Jacobs, Maastricht 2019}

For all articles published, the copyright has been transferred to the respective publisher. No part of this thesis may be reproduced in any form or by any means without written permission from the author or, when appropriate, from the publisher.

\section{Online link:}

https://epubs.ogc.nl/?epub=e.jacobs 


\title{
Spinal Aging
}

\author{
Proefschrift
}

ter verkrijging van de graad van doctor

aan de Universiteit Maastricht,

op gezag van de Rector Magnificus, Prof. dr. Rianne M. Letschert, volgens het besluit van het College van Decanen,

in het openbaar te verdedigen op vrijdag 1 november 2019

om 14.00 uur

door

\section{Eva Jacobs}

Geboren op 22 mei 1989 te Weert 


\section{Promotor}

Prof. dr. L.W. van Rhijn

\section{Copromotoren}

Dr. P.C. Willems

Dr. J.J. Arts

\section{Beoordelingscommissie}

Prof. dr. R.A. de Bie (voorzitter)

Prof. dr. M. de Kleuver (Radboud Universitair Medisch Centrum Nijmegen)

Prof. dr. F.C. Öner (Universitair Medisch Centrum Utrecht)

Dr. J. Sieben

Prof. dr. R.J.E.M. Smeets 
Voor Kristy en Sjors,

Voor altijd in mijn hart. 



\section{CONTENT}

Chapter 1 General Introduction and Thesis Outline

Chapter 2 Gait in patients with symptomatic osteoporotic vertebral

Jacobs E, Senden R, McCrum C, van Rhijn LW, Meijer K, Willems PC

Aging Clin Exp Res. 2019 Apr 27

Chapter 3 The effect of a semirigid thoracolumbar orthosis on gait and sagittal alignment in patients with an osteoporotic vertebral compression fracture

Jacobs E, Senden R, McCrum C, van Rhijn LW, Meijer K, Willems PC

Clin Interv Aging. 2019 Apr 11;14:671-680

Chapter 4 Synthesis and characterization of a new vertebroplasty cement based on gold-containing PMMA microspheres

Jacobs E, Saralidze K, Roth AK, Jong JJA, van den Bergh JPW, Knetsch MLW, Brans B, Lataster A, Dkordevic I, Willems PCPH, Koole LH

Biomaterials. 2016; 82: 60-70

Chapter 5 Reduction of intradiscal pressure by the use of polycarbonateurethane rods as compared to titanium rods in posterior thoracolumbar spinal fixation

Jacobs E, Roth AK, Arts JJ, van Rhijn LW, Willems PC J Mater Sci Mater Med. 2017 Aug 21;28(10):148 
Chapter 6 The implementation of patient reported outcome measures in appropriateness criteria of surgery for degenerative lumbar scoliosis Jacobs E, van Kuijk SMJ, Merk JMR, Vandewall-Peeters M, Jütten-Brouwer LMC, van Rhijn LW, Willems PC

Spine J. 2019 Apr;19(4):655-661

Chapter 7 Prediction of mechanical complications in adult spinal deformity 121 surgery - The GAP score versus the Schwab classification

Jacobs E, van Royen BJ, van Kuijk SMJ, Merk, JMR, Stadhouder A, van Rhijn LW, Willems PC

Spine J. 2019 May;19(5):781-788

Chapter 8 General Discussion

Valorization

Chapter 9 Summary

159

Nederlandse Samenvatting 162

List of Abbreviations 166

Chapter 10 Dankwoord 171

Curriculum Vitae 178

List of Publications 179

List of Presentations 181 




Spinal aging, in terms of osteoporosis, degenerative changes and deformity, will become a major challenge to the spinal community regarding evaluation and treatment for the coming decades. In this thesis various aspects of spinal aging will be clarified.

\section{EPIDEMIOLOGY}

For the upcoming decades, it is forecasted that Europe will experience considerable growth in its aging population (Figure 1). The old-age dependency ratio (people aged 65 and above, relative to those aged 15 to 64) in the European Union is projected to increase by 21.6 purchasing power standards (pps.), from $29.6 \%$ in 2016 to $51.2 \%$ in 2070 [1]. This increase is mainly driven by the very old-age dependency ratio (people aged 80 and above, relative to those aged 15 to 64$)$ which is projected to rise by 14 pps. $(8.3 \%$ to 22.3\%) over this horizon. This shift in the age structure of our Western population is due to decreasing fertility rates, a decreased number of desired offspring and increasing longevity [2].

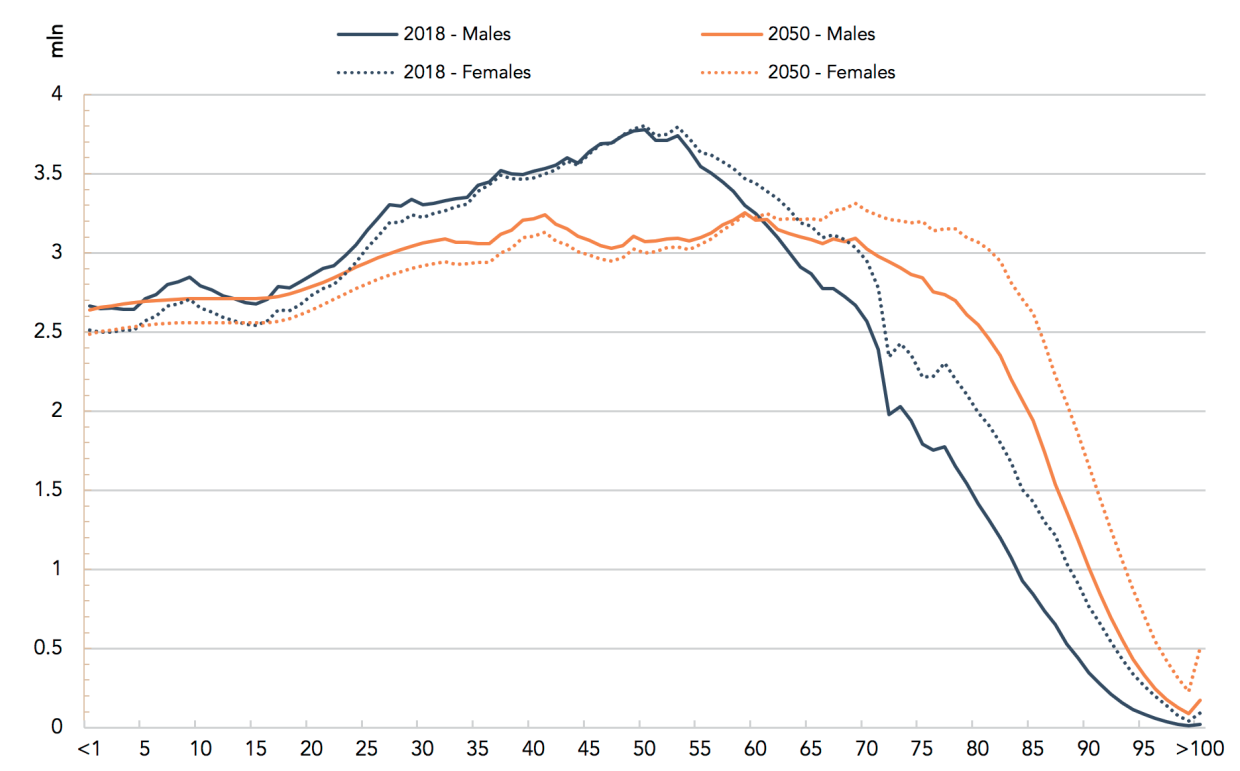

Figure 1. The distribution of the European population over the various age categories for 2018 and 2050. The graphs demonstrate a significant increase in Europe's population above 65 years of age in 2050. 
Aging is characterized by a progressive loss of physiological integrity and represents a decreased capacity for regeneration and repair, leading to impaired function and finally leading to death [2]. Examples of aging-associated diseases are atherosclerosis and cardiovascular disease, hypertension, cancer, arthritis, cataract, osteoporosis, type 2 diabetes and Alzheimer's disease [3]. The incidences of all these diseases increase rapidly with aging. Spinal disorders are one of the most common disabling conditions in our society, posing significant economic costs to society by utilization of healthcare resources and indirect costs by productivity losses $[4,5]$. The Global Burden of Disease Program studied the worldwide impact of disease on health status and disability [6]. The study demonstrated that musculoskeletal conditions such as back pain and arthritis are the second greatest cause of disability, affect more than 1.7 billion people worldwide, and have a major impact on the overall health of the world population [6]. Of all 291 conditions studied, the highest disability measured by patient health status preferences was due to low back pain [7]. Subsequently, disorders of the spine have a significant and measurable impact on health-related quality of life, including pain, function, self-image, mental health, work status, and disability [6, 7].

\section{SPINAL ANATOMY AND NATURAL AGING OF THE SPINE}

The spinal column provides the main musculoskeletal axis of support for the human body, provides mobility at the segmental level and protects the spinal cord from injury [8]. There are seven cervical, twelve thoracic, five lumbar, five sacral and three to four coccygeal vertebrae. A typical vertebra consists of a vertebral body, which is the major weight-bearing component anteriorly, and a vertebral arch. Fibrocartilaginous intervertebral discs separate the vertebral bodies of adjacent vertebrae. The vertebral arch is firmly anchored to the posterior surface of the vertebral body by two pedicles. The roof of the vertebral arch is formed by the laminae. The vertebral arches of the vertebrae are aligned to form the lateral and posterior walls of the vertebral canal, which extends from the first cervical vertebra to the last sacral vertebra. This bony canal contains and protects the spinal cord. The vertebral arch of each vertebra has a spinous process and two transverse processes that serve as attachments for muscles and ligaments and sites of articulation with adjacent vertebrae. A functional spine unit consists of two adjacent vertebrae, the intervertebral disc and adjoining ligaments. It is the smallest physiological motion unit of the spine that exhibits biomechanical characteristics similar to those of the entire spine. The 31 pairs of spinal nerves are segmental in distribution and emerge from the vertebral canal through an intervertebral foramen. Physiologically, in the sagittal plane, the spine has a natural S-shaped curvature. The cervical and lumbar regions have a slight concave curve (lordosis) and the thoracic and sacral regions have a gentle convex curve (kyphosis) with the purpose to maintain mechanical balance in 
the sagittal and coronal plane [8]. A standing posture with minimal muscular energy expenditure is achieved with physiologic curvatures of the spine in the sagittal plane, a straight spine in the coronal plane, a balanced tension of the spinal ligaments, and activation of intrinsic anterior and posterior musculature [9]. Aging of the spine will lead to degenerative alterations starting with subtle biochemical changes, followed by microstructural and eventually gross structural changes of the functional spinal unit. Due to biomechanical consequences the degenerative cycle will progressively modify the functional anatomy. All elements of the spine such as the intervertebral discs, facet joints, vertebral bodies and the supporting muscles and ligaments are affected by this natural aging process [8]. Degeneration of the spine already starts during the first decade of life with biomechanical changes in the disc followed by macroscopic alterations including tears and fissures, which may lead to disc herniation. Secondary to disc degeneration are facet joint changes, including subluxation, cartilage alteration and osteophytosis. Facet hypertrophy and laxity, disc degeneration and enlargement of the ligamentum flavum progressively results in narrowing of the spinal canal as well as in instability such as de novo scoliosis or degenerative spondylolisthesis. With advancing age, osteoporosis may weaken the vertebral bodies, facilitate bone remodeling and rotatory deformities. All combined, aging of the spine can cause rotatory scoliosis, destabilization and rupture of equilibrium [8]. It should be noted that whereas nearly all elderly subjects will exhibit spinal degeneration, many of them will be asymptomatic [10].

Spinal pathology in the elderly characteristically encompasses degenerative pathology, including lumbar spondylosis, stenosis, and instability in the form of segmental instability or deformity; (insufficiency) fractures due to osteoporosis; and spinal metastases [4]. With the aging population, clinicians will be required to manage an increasing number of spinal disorders specific to the elderly, which poses us for important challenges in treatment because this patient category is associated with multiple medical comorbidities, decreased mobility, poor balance, poor bone quality and a greater propensity to falling. When designing and implementing therapeutic strategies, clinicians must consider all of these factors to ensure adequate patient support, optimize outcomes and prevent catastrophic events.

\section{OSTEOPOROSIS AND OSTEOPOROTIC VERTEBRAL COMPRESSION FRACTURES}

Osteoporosis is very common in the aging population affecting millions of people worldwide, predominantly post-menopausal women [11]. It is characterized by a reduced bone mass combined with a disruption of the architecture of the trabecular bone resulting in an increased propensity to fractures [12]. Annually, in Europe, osteoporotic fractures account for the loss of two million Disability Adjusted Life Years (DALYs), which is more 
than accounted for by, e.g., rheumatoid arthritis or hypertensive heart disease [11]. The pathogenesis of osteoporosis is complex and multifactorial [12]. Many osteoporotic patients suffer fractures after minimal trauma, which is pathognomonic of the skeletal fragility caused by low bone mass. One of the most common fractures are osteoporotic vertebral compression fractures (OVCFs), with an estimated number of 500,000 new fractures occurring every year in Europe [13,14]. Although OVCFs can be asymptomatic, most patients experience substantial pain and suffering, significantly decreasing a patient's mobility and quality of life [14]. After an incident vertebral fracture there is a $20 \%$ risk of an additional fracture occurring within the next year [15]. When fractured, the vertebra is commonly deformed by disproportionate height loss from the anterior vertebral body resulting in wedging $[16,17]$. Wedge accumulation over multiple thoracolumbar levels may lead to subsequent spinal deformity, causing an increased thoracolumbar kyphosis and decreased lumbar lordosis (Figure 2) [18].

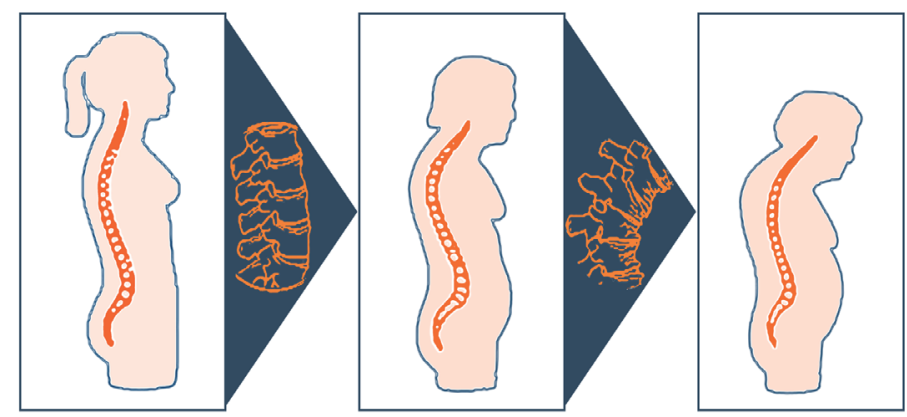

Figure 2. Wedge accumulation over multiple levels leading to thoracolumbar hyperkyphosis.

The increased anterior spinal loading in degenerative thoracolumbar hyperkyphosis has been associated with a downward spiral of additional vertebral compression fractures, also known as the "vertebral fracture cascade" [19]. Thoracolumbar hyperkyphosis may negatively impact several aspects of an afflicted individual's health including physical function, pulmonary function, pain and disability, postural control during walking, and even mortality (Figure 3) [19, 20]. 


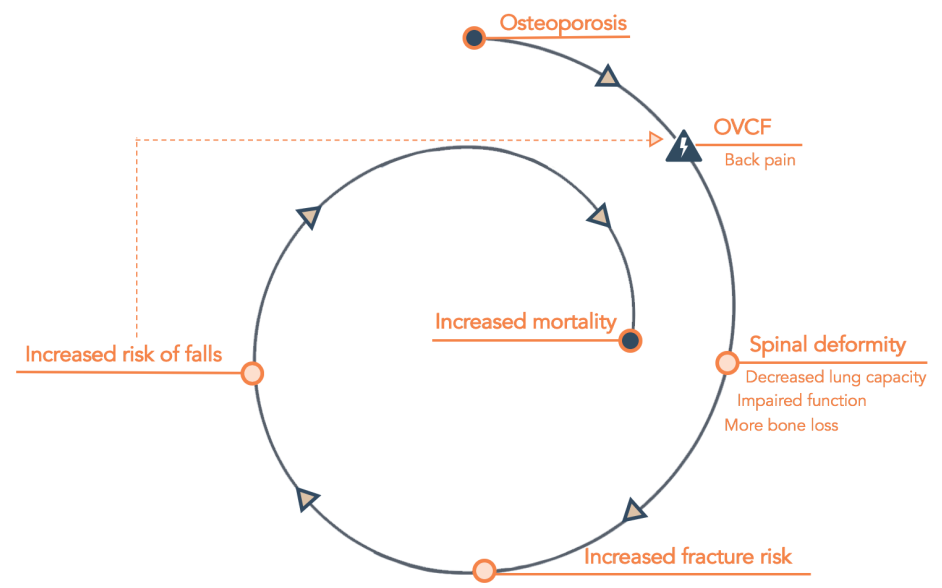

Figure 3. The downward spiral after an osteoporotic vertebral compression fracture [19].

Treatment of OVCFs should aim to break the downward spiral of recurrent vertebral compression fractures and to prevent the subsequent progression of kyphosis and resultant global sagittal malalignment. Furthermore, it should intend to prevent or slow down the decline in postural control, thereby limiting the increased risk of falling in these patients.

\section{DEGENERATIVE SPINAL DEFORMITY}

Degenerative spinal deformity or adult spinal deformity (ASD) can be defined as an abnormality in alignment or curvature of one or more portions of the spine [21]. Such deformities can involve any combination of abnormal alignment in the axial, coronal and sagittal plane $[22,23]$. Degenerative spinal deformity afflicts a significant portion of the elderly, and is increasing in prevalence [24]. It comprises a wide range of conditions that result in abnormal spinal alignment and may result in pain, disability, neurological impairment, and/or loss of function. In middle-aged and older adults, the most prevalent deformities are de novo degenerative lumbar scoliosis (DLS), the aforementioned thoracolumbar hyperkyphosis, and to a lesser extent iatrogenic deformities. The etiology is complex, multifactorial, and is associated with progressive and asymmetric degeneration of the lumbar discs, facet joints, and other structural spinal elements typically leading to rotational deformity and possibly neural element compression [22]. The most frequent symptom in patients suffering from degenerative spinal deformity is axial back pain, which may be accompanied by radicular leg pain or neurogenic claudication symptoms. Other symptoms include stiffness, muscular fatigue, neurological deficits in the lower extremities, and occasionally cauda equine syndrome [25]. Degenerative spinal 
deformity adversely affects health-related quality of life and has become a considerable healthcare concern [26, 27].

Central to the current understanding of degenerative spinal deformity and its management is the concept of global spinal alignment. The load distribution on the spine depends more on its shape and curvature in the sagittal plane than in the coronal plane [28]. Global spinal alignment serves to maintain an upright posture and horizontal gaze while minimizing muscle energy expenditure and is dependent on the interaction between regional spinal and pelvic alignment [21]. In order to achieve an ergonomic standing posture the centre of mass (CoM) should be positioned over the feet (base of support, BoS). In the normal population, there is no standard sagittal balance because it is driven by pelvic morphology and varies with age and gender [29]. In a healthy spine the center of rotation is positioned

within the vertebral bodies. In this case, a relaxed upright posture only costs $7 \%$ more energy than supine relaxing. However, only small postural changes can already change the load distribution of the spine (Figure 4).

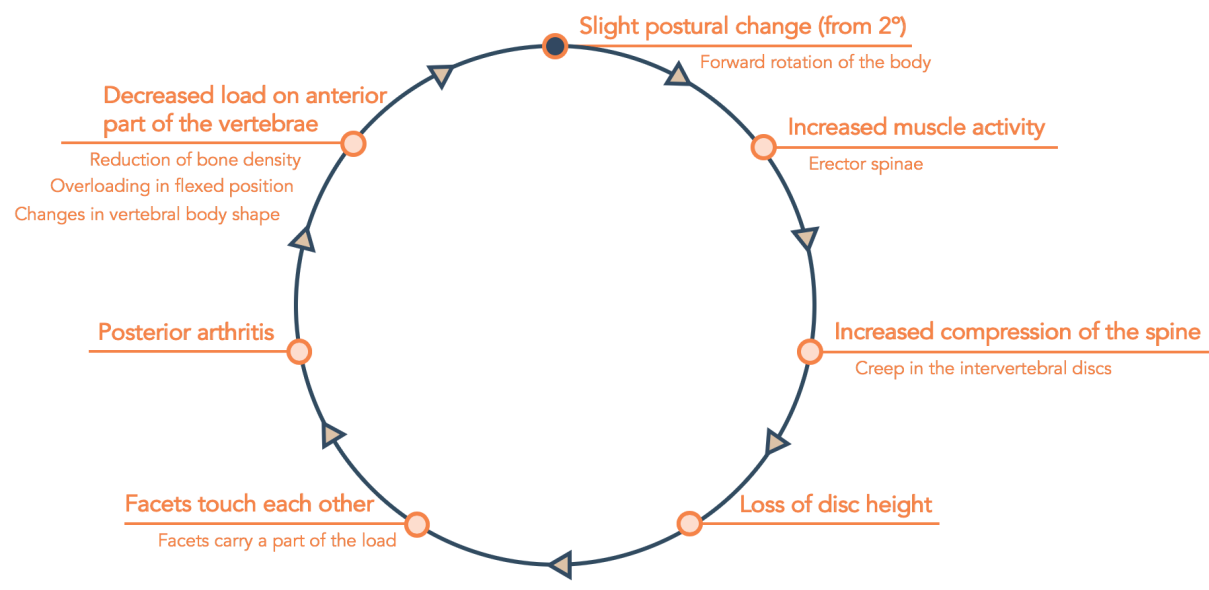

Figure 4. The effect of small postural changes on spinal loading [30]. Reproduced with permission of dr. V. Lafage, Hospital for Special Surgery, New York City.

As a consequence, the degenerative changes in degenerative spinal deformity have the potential to greatly disrupt the normal curvature of the spine, leading to sagittal malalignment with resulting higher loading forces and energy expenditure (Figure 4 and 5) [30]. 


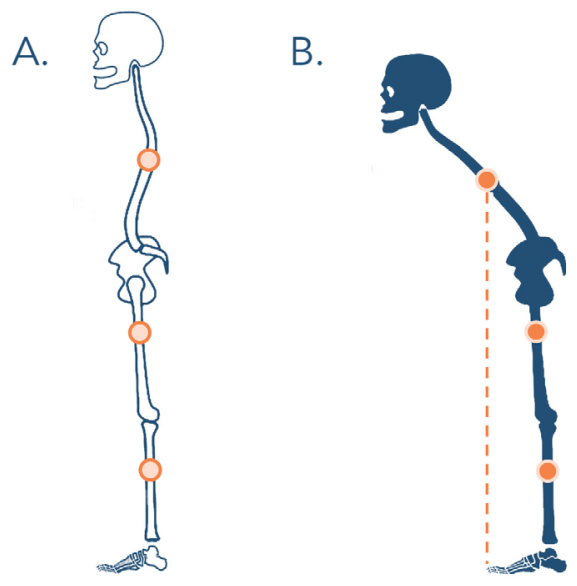

Figure 5. A. Normal sagittal alignment in which the gravity line is positioned between the feet. B. Sagittal malalignment in which the gravity line is positioned anteriorly to the feet. Reproduced with permission of dr. V. Lafage, Hospital for Special Surgery, New York City.

As a response to positive sagittal (mal)alignment, the body progressively recruits compensatory mechanisms to counteract the anterior truncal shift (Figure 6) [30]. A chain of compensation is initiated from the flexible parts of the spine, and extends to the hips, lower extremities, and cervical spine to preserve an erect posture and horizontal gaze. The two most important mechanisms to compensate for this are the pelvic retroversion (in order to maintain a vertical trunk) and the pelvic shift (in order to translate the centre of mass posteriorly again) (Figure 6). Compensation mechanisms vary among pa-

A.

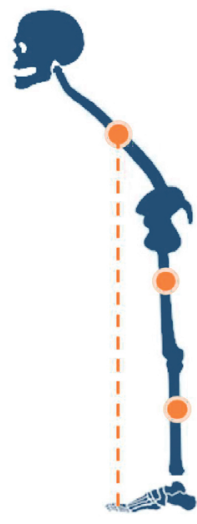

B.

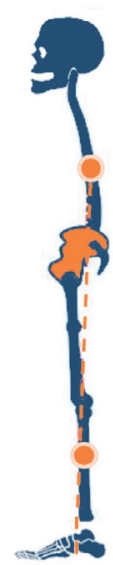

c.

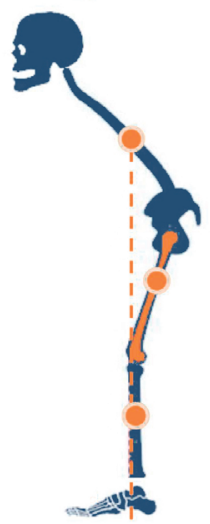

Figure 6. A. Sagittal malalignment without compensation mechanisms. B. Pelvic retroversion in order to maintain the trunk vertical. C. Pelvic shift in order to translate the CoM posteriorly. Reproduced with permission of dr. V. Lafage, Hospital for Special Surgery, New York City. 
tients depending on their ability to compensate (based on flexibility, condition etcetera). Younger patients tend to mainly flatten their thoracic spine as an early response whereas older patients prefer the pelvic lower limb mechanics. Females tend to extend the hips and increase their pelvic tilt whereas men demonstrate a higher degree of knee flexion independent from the pelvic tilt. The interaction between deformity and compensatory mechanisms depicts the final presentation of patients with degenerative spinal deformity [31].

\section{FALLS IN THE ELDERLY}

The prevalence of falls among elderly people is high: at least $30-40 \%$ of patients aged over 65 experience one or more fall accidents annually [32]. There are many distinct causes for falls in the elderly, such as balance and gait disorders, poor vision, polypharmacy and environmental factors. Balance is a multidimensional concept, referring to the ability of a person not to fall [33]. The ability to maintain, achieve or restore a state of balance during any posture or activity is called "postural control". Postural control can be defined as the maintenance of the body centre of mass (CoM) over its base of support (BoS) (or more generally, within the limits of stability) [34]. Adequate postural control is essential for daily activities, and requires integration of visual, proprioceptive and vestibular information [35]. It has been shown that spinal imbalance, especially global sagittal malalignment, causes impaired postural control, slower gait and a wider base of support with stance and gait [17, 36, 37]. Impaired postural control during walking is a major risk factor for falls [36, 37]. Falls among the elderly who are frail and have osteoporosis cause a tremendous amount of morbidity, mortality and use of healthcare services including premature nursing home admissions [11, 38].

\section{THESIS OUTLINE}

This thesis on spinal aging consists of two major parts. The first part (Chapter 2-5) focuses on osteoporotic vertebral compression fractures (OVCFs) and its consequences; the second part (Chapter $\mathbf{6}$ and 7) focuses on degenerative spinal deformity. The following questions were postulated:

\section{Are spatiotemporal gait parameters affected in patients with an OVCF as compared to healthy controls?}

As mentioned previously, one factor related to disability in people with spinal deformity is decreased postural control and increased risk of falling [36, 37]. However, in literature, 
little is known about the effect of vertebral fractures on gait and stability. Walking characteristics of elderly with and without vertebral fractures have not yet been evaluated [36]. Therefore, the impact of OVCFs on gait stability was examined in Chapter 2 and compared to gait stability in healthy participants. Furthermore, this prospective cohort study aimed to examine the spatiotemporal gait parameters in patients suffering an OVCF over six months following the fracture. It was hypothesized that the gait pattern would be more variable in people with an osteoporotic vertebral fracture and that stability would be reduced compared with healthy participants. Regarding the longitudinal data it was hypothesized that the gait pattern and stability would return to normal over time.

\section{What is the effect of a thoracolumbar dynamic orthosis on gait in patients suffering from an OVCF?}

Primarily, treatment of OVCFs should intend to relief pain, slow down the decline in postural control and limit the risk of falls and further fractures in this frail patient group. A second important goal in the treatment of OVCFs is the prevention of the subsequent progression to hyperkyphosis and global sagittal malalignment. Current conservative treatment of symptomatic OVCFs is multimodal and comprises analgesics, medication for osteoporosis and physical therapy. However, although widely used in current daily practice, little is known about the value of orthoses in the treatment of osteoporotic vertebral compression fractures. In Chapter $\mathbf{3}$ the potential role of wearing a semirigid thoracolumbar orthosis in the conservative treatment of OVCFs is evaluated in an observational study. The primary purpose of this study was to examine the direct effect of a thoracolumbar dynamic brace on gait in patients suffering from an OVCF. The secondary purpose was to evaluate changes in gait, radiographic sagittal alignment, pain and quality of life over time.

3. What are the effects on doughing time, injection time, cytocompatibility and mechanical properties of incorporating gold particles in polymethylmethacrylate (PMMA) microspheres as radiopacifier instead of barium sulphate in cement for vertebroplasty?

With natural healing of the acute fracture, the initial pain caused by the fracture subsides in a majority of patients, usually within a couple of weeks [39]. However, up to one third of patients experience insufficient response and/or intolerance to conservative treatment of OVCFs [40]. In those cases interventional treatment may be considered. Percutaneous vertebroplasty is a minimally invasive procedure in which bone cement is carefully injected into a painful osteoporotic fractured vertebra under continuous fluoroscopic guidance. The bone cement hardens in situ, providing increased strength 
and stabilization. Most importantly, pain is substantially relieved which, results in a significant improvement of quality of life in the elderly population. For radiopacity, barium sulphate $\left(\mathrm{BaSO}_{4}\right)$ is added to the PMMA bone cement. However, there are a number of drawbacks of incorporating large concentrations of $\mathrm{BaSO}_{4}$ as the radiopacifier in PMMA-based bone cements for percutaneous vertebroplasty. These include adverse effects on injectability, viscosity profile, setting time, mechanical properties of the cement and bone resorption. In Chapter 4, a novel cement, designed to address some of these drawbacks, is studied.

\section{Does rod stiffness have an effect on intraspinal loading forces after posterior pedicle screw fixation?}

As stated before, ultimately, treatment of OVCF should aim to breakdown the vicious cycle known as the "vertebral fracture cascade" (Figure 3). Loss of sagittal alignment and balance in adult spinal deformity can cause severe pain, disability and progressive neurological deficit. When conservative treatment has failed, spinal fusion using rigid instrumentation is currently the salvage treatment to stop further curve progression in thoracolumbar hyperkyphosis. However, fusion surgery is associated with high revision rates due to instrumentation failure and proximal junctional failure, especially if patients suffering from osteoporosis. To address these drawbacks, in Chapter $\mathbf{5}$ a less rigid rod construct is proposed, which is hypothesized to provide a more gradual transition of force and load distribution over spinal segments in comparison to stiff titanium rods. In this pilot study, the effect of variation in rod stiffness on the intradiscal pressure (IDP), indicative of intraspinal loading forces, of fixed and non-fixed spinal segments during flexion-compression loading was assessed.

Chapter 6 and Chapter 7 elaborate on how to guide decision-making for well-defined populations of patients suffering from degenerative spinal deformity using algorithms based on a combination of the best available evidence and multidisciplinary expert opinion.

\section{To what extent is the patient perspective incorporated into the appropriate- ness criteria of surgery for degenerative lumbar scoliosis?}

Degenerative lumbar scoliosis (DLS) is an increasingly common spinal disorder of which current management is characterized by a substantial variety in treatment advice [25]. To improve evidence-based clinical decision-making and increase uniformity and transparency of care, the Scoliosis Research Society (SRS) established appropriateness criteria for surgery for DLS [25]. In these criteria, however, the patient perspective was not formally incorporated. Since patient perspective is an increasingly important consideration in informed decision-making, embedding Patient Reported Outcome Measures (PROMs) in the appropriateness criteria would allow for a more objective and transparent patient- 
centered approach. In Chapter 6, the extent that patient perspective is integrated into the appropriateness criteria of surgery for DLS is evaluated in a retrospective cohort study.

\section{What classification system is the most appropriate for the prediction of me- chanical failure after adult spinal deformity surgery?}

Surgery for degenerative spinal deformity is a challenging and complex procedure with high reported complication (8.4-42\%) and revision rates (9-17.6\%) [31, 41, 42]. Failure to achieve or maintain adequate postoperative sagittal alignment has been reported to be the main cause of mechanical complications. In order to define appropriate surgical targets the Scoliosis Research Society (SRS)-Schwab classification and the Global Alignment and Proportion (GAP) score were established [28]. In literature, no study has yet compared these classification systems with respect to the risk of developing mechanical complications. In Chapter 7, the ability of the Schwab classification and the GAP score to predict mechanical complications following adult spinal deformity surgery is assessed and compared in a retrospective cohort study.

Finally, Chapter $\mathbf{8}$ comprises a discussion of the main findings of the previous chapters, addresses the main limitations of the studies, provides final conclusions and recommendations, and includes opportunities for future research. 


\section{REFERENCES}

1. The 2018 Ageing Report: Economic and Budgetary Projections from the 28 EU Member States (2016-2070). 2018.

2. Fehlings MG, et al. The Aging of the Global Population: The Changing Epidemiology of Disease and Spinal Disorders. Neurosurgery, 2015. 77 Suppl 4: p. S1-5.

3. Kalache A and I Keller. The greying world: a challenge for the twenty-first century. Sci Prog, 2000. 83 ( Pt 1): p. 33-54.

4. Waldrop R, et al. The Burden of Spinal Disorders in the Elderly. Neurosurgery, 2015. 77 Suppl 4: p. S46-50.

5. Weinstein SL. 2000-2010: the bone and joint decade. J Bone Joint Surg Am, 2000. 82(1): p. 1-3.

6. Murray CJ, et al. Disability-adjusted life years (DALYs) for 291 diseases and injuries in 21 regions, 1990-2010: a systematic analysis for the Global Burden of Disease Study 2010. Lancet, 2012. 380(9859): p. 2197-223.

7. Hoy D, et al. Measuring the global burden of low back pain. Best Pract Res Clin Rheumatol, 2010. 24(2): p. 155-65.

8. Benoist M. Natural history of the aging spine. Eur Spine J, 2003. 12 Suppl 2: p. S86-9.

9. Schwab F, et al. Adult spinal deformity-postoperative standing imbalance: how much can you tolerate? An overview of key parameters in assessing alignment and planning corrective surgery. Spine (Phila Pa 1976), 2010. 35(25): p. 2224-31.

10. Boden SD, et al. Abnormal magnetic-resonancescans of the lumbar spine in asymptomatic subjects. A prospective investigation. J Bone Joint Surg Am, 1990. 72(3): p. 403-8.

11. Hernlund E, et al. Osteoporosis in the European Union: medical management, epidemiology and economic burden. A report prepared in collaboration with the International Osteoporosis Foundation (IOF) and the European Federation of Pharmaceutical Industry Associations (EFPIA). Arch Osteoporos, 2013. 8: p. 136.

12. Rosen CJ. Pathogenesis of osteoporosis. Baillieres Best Pract Res Clin Endocrinol Metab, 2000. 14(2): p. 181-93.

13. Johnell $\mathrm{O}$ and JA Kanis. An estimate of the worldwide prevalence and disability associated with osteoporotic fractures. Osteoporos Int, 2006. 17(12): p. 1726-33.

14. Luthman S, J Widen, and F Borgstrom. Appropriateness criteria for treatment of osteoporotic vertebral compression fractures. Osteoporos Int, 2018. 29(4): p. 793-804.

15. Lindsay R, et al. Risk of new vertebral fracture in the year following a fracture. JAMA, 2001. 285(3): p. 320-3.

16. Luo J, et al. Vertebral deformity arising from an accelerated "creep" mechanism. Eur Spine J, 2012. 21(9): p. 1684-91.

17. Katzman WB, et al. Age-related hyperkyphosis: its causes, consequences, and management. J Orthop Sports Phys Ther, 2010. 40(6): p. 352-60.

18. Wei Y, et al. Thoracolumbar kyphosis is associated with compressive vertebral fracture in postmenopausal women. Osteoporos Int, 2017. 28(6): p. 1925-1929.

19. Broy SB. The Vertebral Fracture Cascade: Etiology and Clinical Implications. J Clin Densitom, 2016. 19(1): p. 29-34.

20. Ailon T, et al. Progressive Spinal Kyphosis in the Aging Population. Neurosurgery, 2015. 77 Suppl 4: p. S164-72.

21. Ailon T, et al. Degenerative Spinal Deformity. Neurosurgery, 2015. 77 Suppl 4: p. S75-91. 
22. Benner B and G Ehni. Degenerative lumbar scoliosis. Spine (Phila Pa 1976), 1979. 4(6): p. 548-52.

23. Aebi M. The adult scoliosis. Eur Spine J, 2005. 14(10): p. 925-48.

24. Kotwal S, et al. Degenerative scoliosis: a review. HSS J, 2011. 7(3): p. 257-64.

25. Chen PG, et al. Surgery for Degenerative Lumbar Scoliosis: The Development of Appropriateness Criteria. Spine (Phila Pa 1976), 2016. 41(10): p. 910-8.

26. Glassman SD, et al. The costs and benefits of nonoperative management for adult scoliosis. Spine (Phila Pa 1976), 2010. 35(5): p. 578-82.

27. Yamada K, et al. Targeted Therapy for Low Back Pain in Elderly Degenerative Lumbar Scoliosis: A Cohort Study. Spine (Phila Pa 1976), 2016. 41(10): p. 872-9.

28. Yilgor C, et al. Global Alignment and Proportion (GAP) Score: Development and Validation of a New Method of Analyzing Spinopelvic Alignment to Predict Mechanical Complications After Adult Spinal Deformity Surgery. J Bone Joint Surg Am, 2017. 99(19): p. 1661-1672.

29. Lafage R, et al. Defining Spino-Pelvic Alignment Thresholds: Should Operative Goals in Adult Spinal Deformity Surgery Account for Age? Spine (Phila Pa 1976), 2016. 41(1): p. $62-8$.

30. Barrey C, et al. Sagittal balance disorders in severe degenerative spine. Can we identify the compensatory mechanisms? Eur Spine J, 2011. 20 Suppl 5: p. 626-33.

31. Diebo BG, et al. Sagittal deformities of the spine: factors influencing the outcomes and complications. Eur Spine J, 2015. 24 Suppl 1: p. S3-15.

32. Rubenstein LZ. Falls in older people: epidemiology, risk factors and strategies for prevention. Age Ageing, 2006. 35 Suppl 2: p. ii37-ii41.

33. Pollock AS, et al. What is balance? Clin Rehabil, 2000. 14(4): p. 402-6.

34. Alexander NB. Postural control in older adults. J Am Geriatr Soc, 1994. 42(1): p. 93-108.

35. Lamoth CJ and MJ van Heuvelen. Sports activities are reflected in the local stability and regularity of body sway: older ice-skaters have better postural control than inactive elderly. Gait Posture, 2012. 35(3): p. 489-93.

36. de Groot $\mathrm{MH}$, et al. A flexed posture in elderly patients is associated with impairments in postural control during walking. Gait Posture, 2014. 39(2): p. 767-72.

37. van der Jagt-Willems HC, et al. Associations between vertebral fractures, increased thoracic kyphosis, a flexed posture and falls in older adults: a prospective cohort study. BMC Geriatr, 2015. 15: p. 34.

38. Sinaki M, et al. Balance disorder and increased risk of falls in osteoporosis and kyphosis: significance of kyphotic posture and muscle strength. Osteoporos Int, 2005. 16(8): p. 1004-10.

39. Venmans A, et al. Natural history of pain in patients with conservatively treated osteoporotic vertebral compression fractures: results from VERTOS II. AJNR Am J Neuroradiol, 2012. 33(3): p. 519-21.

40. Lee HM, et al. Comparative analysis of clinical outcomes in patients with osteoporotic vertebral compression fractures (OVCFs): conservative treatment versus balloon kyphoplasty. Spine J, 2012. 12(11): p. 998-1005.

41. Schwab FJ, et al. Risk factors for major peri-operative complications in adult spinal deformity surgery: a multi-center review of 953 consecutive patients. Eur Spine J, 2012. 21(12): p. 2603-10.

42. Bianco K, et al. Complications and intercenter variability of three-column osteotomies for spinal deformity surgery: a retrospective review of 423 patients. Neurosurg Focus, 2014. 36(5): p. E18. 



\section{PART I}

\section{Osteoporotic Vertebral Compression Fractures}





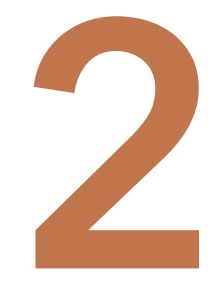

\section{Gait in patients with symptomatic osteoporotic vertebral compression fractures over six months} recovery

Jacobs E, Senden R, McCrum C, van Rhijn LW, Meijer K, Willems PC Aging Clin Exp Res. 2019 Apr 27 


\section{ABSTRACT}

Background - One factor related to disability in people with spinal deformity is decreased postural control and increased risk of falling. However, little is known about the effect of osteoporotic vertebral compression fractures (OVCFs) and their recovery on gait and stability. Walking characteristics of older adults with and without vertebral fractures have not yet been compared.

Aims - The purpose of the current study was to examine the spatiotemporal gait parameters and their variability in patients with an OVCF and healthy participants during treadmill walking at baseline and after six months of recovery.

Methods - Twelve female patients suffering a symptomatic OVCF were compared to eleven matched controls. Gait analysis was performed with a dual belt instrumented treadmill with a 180 degrees projection screen providing a virtual environment (Computer Assisted Rehabilitation Environment). Results of patients with an OVCF and healthy participants were compared. Furthermore, spatiotemporal gait parameters were assessed over six months following the fracture.

Results - Patients suffering from an OVCF appeared to walk with significantly shorter, faster and wider strides as compared to their healthy counterparts. Although stride time and length improved over time, the majority of the parameters analyzed remained unchanged after six months of conservative treatment.

Discussion - Since patients do not fully recover to their previous level of mobility after six months of conservative treatment for OVCF, it appears of high clinical importance to add balance and gait training to the treatment algorithm of OVCFs.

Conclusions - Patients suffering from an OVCF walk with shorter, faster and wider strides as compared to their healthy counterparts and adopt a less stable body configuration in the anterior direction, potentially increasing their risk of forward falls if perturbed. Although stride time and stride length improve over time even reaching healthy levels again, patients significantly deviate from normal gait patterns (e.g. in stability and step width) after six months of conservative treatment. 


\section{INTRODUCTION}

One of the most common fractures among older adults are osteoporotic vertebral compression fractures (OVCFs), with an estimated 500,000 new fractures occurring every year in Europe [1, 2]. After an OVCF there is disproportionate height loss from the anterior vertebral body resulting in a wedge shaped vertebra [3]. The presence of an OVCF is a strong predictor for subsequent vertebral fractures [4]. Wedge accumulation over multiple thoracolumbar levels may lead to thoracolumbar hyperkyphosis. Subsequent sagittal malalignment with inability to stand upright is the strongest driver of pain and disability in older adults suffering from spinal deformity $[5,6]$. In the majority of the patients the initial pain caused by the fracture usually subsides within a couple of weeks, with healing of the fracture [2]. However, up to one third of patients experience incapacitating pain for months to years due to insufficient response to treatment $[2,7]$.

One factor related to disability in people with spinal deformity such as thoracolumbar hyperkyphosis is decreased postural control and an increased risk of falling [8, 9]. Falls among older adults, especially those with osteoporosis, are associated with high morbidity, even mortality and involve high-costs for society [8-10]. De Groot et al. [8] described that patients with an OVCF and thoracolumbar hyperkyphosis demonstrate impaired postural control. This may be due to the fact that in thoracolumbar hyperkyphosis, there is an increased forward bending moment due to the forward curvature of the trunk, which shifts the body's centre of mass forward relative to the centre of rotation, i.e. the spine. In this situation, it may be more difficult to keep the centre of mass within the base of support (a requirement for stability in static situations) [11]. However, as the majority of falls in older adults occurs during walking, stability control specifically while walking should be considered. It is important to note that stability control during static tasks show little relationship with more dynamic and reactive tasks [12-14]. In dynamic situations such as walking, the velocity of the centre of mass must be accounted for when assessing stability [11, 15]. For this purpose, Hof et al. [11] proposed the extrapolated centre of mass concept, in which the position and velocity of the centre of mass are taken into account when determining the stability of the body position. As people with thoracolumbar hyperkyphosis already have a more anterior centre of mass, stability in the anterior direction during walking may be reduced, perhaps increasing the difficulty in coping with forward losses of balance (e.g. trips) [8, 9]. Gait variability is another quantifiable feature of walking that is altered in clinically relevant syndromes or symptoms, such as falling, frailty and neuro-degenerative diseases. In literature it has been demonstrated that increased gait variability is associated with an increased risk of falling [16]. However, there is little information available on the effect of OVCF on gait stability and variability.

The current study aimed to examine the spatiotemporal gait parameters and their variability in patients with an OVCF and healthy participants during treadmill walking. Furthermore, this study aimed to monitor recovery of spatiotemporal gait parameters 
and variability in patients suffering an OVCF over six months following the fracture. It was hypothesized that gait would be more variable in people with an osteoporotic vertebral fracture and that stability in the anterior direction would be reduced compared with healthy participants. Regarding the longitudinal data, is was hypothesized that gait would improve over time in terms of variability and stability.

\section{MATERIALS AND METHODS}

\subsection{Participants}

Twelve female patients [mean age 68 (55-78) yrs, mean height $1.61 \mathrm{~m}(1.51-1.73 \mathrm{~m})$, weight $67.34 \mathrm{~kg}(49-88 \mathrm{~kg})]$ with a symptomatic OVCF who presented at the emergency department of the Maastricht University Medical Centre gave written informed consent to participate in this study. Participants were included if they were female, aged 55 years or older and were fully ambulatory (able to perform a $15 \mathrm{~m}$ walk test without assistance). Patients were excluded if the vertebral fracture was unstable and required surgery, if they had neurological deficits, active/recent cancer, a psychiatric diagnosis, a history of neurogenic or myopathic disorders impairing sensory or motor function or medication that could affect balance or walking. The study was explained before obtaining informed consent and conducted in accordance with the Declaration of Helsinki and was approved by the Maastricht University Medical Centre medical ethics committee (NL52978.057.15). Patients were treated with an orthosis (Osteolind ${ }^{\circledR}$ plus, Werkmeister, Wanfried, Germany) which they wore all day for the first six weeks. From six weeks to six months wearing the orthosis was optional. Data was collected at baseline, after six weeks and after six months.

A gender, age, height and weight matched control group was selected from the database of a previous study in the Maastricht University Medical Centre (NL58205.068.16) with eleven healthy older female adults [71 years (66-78 yrs), $1.64 \mathrm{~m}$ height (1.58-1.70 m) and $68.5 \mathrm{~kg}(62-81 \mathrm{~kg})$ weight]. The participants had no self-reported history of walking difficulties, dizziness or balance problems, could walk for at least 30 minutes without assistance, without stopping and had no known neuromuscular condition, injury or medication that could affect balance or walking. Unpaired t-tests confirmed that the groups were not significantly different in age, height or weight. Additionally, equivalence tests with $90 \%$ confidence intervals revealed that the group differences were statistically equivalent and not meaningfully different.

\subsection{Equipment}

The measurements were conducted with the Computer Assisted Rehabilitation Environment Extended (CAREN; Motekforce Link, Amsterdam, The Netherlands), including a dual-belt instrumented treadmill (force plates: $1000 \mathrm{~Hz}$ ), a 12 camera Vicon Nexus motion 
capture system (100Hz; Vicon Motion Systems, Oxford, UK) and a virtual environment that provided optic flow. Participants wore a safety harness connected to an overhead frame. Ten retroreflective markers were attached to anatomical landmarks (one acromion marker on each side, four pelvic markers, one marker each lateral distal femur condyle and one marker on each lateral malleolus) and were tracked by the motion capture system. The marker trajectories were filtered using a low pass second order Butterworth filter (zerophase) with a cut-off frequency of $12 \mathrm{~Hz}$. Foot touchdown and toe-off were determined using the treadmill force plates (50N threshold) in combination with a marker-based method [17].

\subsection{Measurement Procedure}

Participants first completed treadmill walking familiarization trials (consisting of 90 seconds walking at $1 \mathrm{~m} / \mathrm{s}$ followed by 90 seconds walking at self-paced speed) after which they continued walking for three minutes at a self-paced mode followed by three minutes at a set speed of $1.0 \mathrm{~m} / \mathrm{s}$. For each of the three minutes, the first 90 seconds was unperturbed walking and the following 90 seconds included five mediolateral platformshift perturbations. The perturbations were part of another study and are not further discussed here. The final 60 strides of the 1-m/s walking trial were taken for further analysis, as well as the average walking speed during the unperturbed 90 seconds selfpaced measurement as a functional outcome. In the OVCF group, the change in pain level was assessed using an 11-point Visual Analog Scale (VAS) where level 10 implies extreme pain and level 0 no pain at all.

\subsection{Healthy Control Data}

Gait data from a walking trial at $1.0 \mathrm{~m} / \mathrm{s}$ was taken for analysis. It is important to note that a slightly different reduced kinematic model was used for these subjects. For these participants, six markers were attached to each hallux, each trochanter major, the sacrum and the C7 vertebra. In order to determine the likely absolute differences of the two marker sets, we conducted a small pilot study with six participants wearing both marker sets. The protocol for these healthy pilot participants, and the details on the marker set comparability pilot study can be found in the supplement. The results section of the current study describes both the absolute data and the results accounting for the absolute differences found between the marker sets.

\subsection{Spatiotemporal Gait and Variability Parameters}

The gait parameters considered were stride time, stride length, step width and double support time. The mean value for each participant was calculated from all recorded steps. Additionally, the coefficient of variation (CV) was calculated for all gait parameters in order to evaluate gait variability. The margins of stability (MoS) was calculated in both the anteroposterior (AP) and mediolateral (ML) directions at foot touchdown as the AP or ML distance between the boundary of the base of support (BoS; the ankle marker) and the 
extrapolated centre of mass (XCoM), as defined by Hof et al. [11]. For the estimation of the centre of mass position and velocity, the average positions of the four pelvis markers were used. For the MoS the averages, medians and standard deviations were used for analysis.

\subsection{Statistics}

Independent t-tests were used to determine if differences existed between healthy control participants and patients with OVCF in all outcome parameters. To detect potential changes in the gait parameters in the patients over time, one way repeated measures ANOVAs with Tukey post hoc tests for multiple comparisons were conducted. Statistical analyses were conducted with GraphPad Prism version 7.03 for Windows (GraphPad Software Inc., La Jolla, California, USA). Significance was set at $\alpha=0.05$.

\section{RESULTS}

\subsection{Baseline}

The results for the spatiotemporal gait parameters at baseline (stride time, stride length, step width, double support time) of the OVCF group and the healthy controls are presented in Table 1 . The independent t-tests revealed a significantly shorter stride time, a significantly shorter stride length and a significantly greater step width for the OVCF group compared to the healthy participants, with no significant difference in double support time (Table 1). Additionally, the independent t-tests revealed significantly lower AP MoS and significantly higher ML MoS for the OVCF group compared to controls (Table 1). All of the above differences exceeded any bias due to the difference in marker sets (Table 1; Supplementary Information). However, it is important to highlight that when accounting for the difference in the marker sets, the AP MoS is estimated to be on average $6 \mathrm{~cm}$ greater for the OVCF group (i.e. more stable anteriorly).

No significant differences in the coefficients of variation for the four spatiotemporal gait parameters were found between the groups (Table 2).

Table 1. Spatiotemporal characteristics and margins of stability during walking at $1 \mathrm{~m} / \mathrm{s}$.

\begin{tabular}{lcccccc}
\hline & Stride & Stride & Step & $\begin{array}{c}\text { Double } \\
\text { support } \\
\text { time (s) }\end{array}$ & $\begin{array}{c}\text { length (m) } \\
\text { time (s) }\end{array}$ & MoS AP (m) MoS ML (m) \\
\hline OVCF & $1.09 \pm 0.05$ & $1.10 \pm 0.05$ & $0.22 \pm 0.03$ & $0.13 \pm 0.02$ & $-0.100 \pm 0.034$ & $0.048 \pm 0.011$ \\
Control & $1.17 \pm 0.08$ & $1.17 \pm 0.08$ & $0.14 \pm 0.03$ & $0.14 \pm 0.02$ & $0.105 \pm 0.032$ & $0.001 \pm 0.009$ \\
P-value & 0.013 & 0.024 & $<0.0001$ & 0.256 & $<0.0001$ & $<0.0001$ \\
$\begin{array}{l}\text { Exceeds bias of } \\
\text { marker set difference? }\end{array}$ & Yes & Yes & Yes & N/A & Yes* & Yes \\
\hline
\end{tabular}

*This difference is higher than the upper confidence limit of the Bland Altman plot, indicating that the OVCF group had a larger MoS AP value by on average $6 \mathrm{~cm}$ if the same marker set would have been used. 
Table 2. Variability in the spatiotemporal characteristics and margins of stability during walking at $1 \mathrm{~m} / \mathrm{s}$.

\begin{tabular}{lcccccc}
\hline & \multicolumn{7}{c}{$\begin{array}{c}\text { Double } \\
\text { support } \\
\text { time } \\
\text { Stride time } \\
(\mathbf{C V})\end{array}$} & $\begin{array}{c}\text { Stride length } \\
(\mathbf{C V})\end{array}$ & $\begin{array}{c}\text { Step width } \\
(\mathbf{C V})\end{array}$ & $\begin{array}{c}\text { (CV) } \\
\text { OVCF }\end{array}$ & $2.09 \pm 0.69$ & $3.49 \pm 2.47$ & $11.87 \pm 8.04$ & $9.39 \pm 3.22$ & $0.019 \pm 0.007$ & $0.014 \pm 0.004$ \\
Control & $1.93 \pm 0.62$ & $2.90 \pm 1.20$ & $16.48 \pm 5.48$ & $10.49 \pm 2.34$ & $0.022 \pm 0.007$ & $0.013 \pm 0.003$ \\
P-value & 0.556 & 0.480 & 0.126 & 0.361 & 0.331 & 0.754 \\
\hline
\end{tabular}

\subsection{Longitudinal Data}

The repeated measures ANOVAs revealed significant effects of time on stride time (F[1.2, $14]=6.4, \mathrm{p}=0.0191$; Figure 1$)$ and stride length $(\mathrm{F}[1.2,13]=4.4, \mathrm{p}=0.0495$; Figure 1$)$ and nonsignificant effects of time on step width $(\mathrm{F}[1.9,21]=2.4, \mathrm{p}=0.1178$; Figure 1$)$, double support time $(F[1.6,17]=1.7, p=0.2182$; Figure 1), $A P \operatorname{MoS}(F[1.3,14]=2.5, p=0.1269$; Figure 2$)$ and $\mathrm{ML}$ $\operatorname{MoS}(\mathrm{F}[1.5,16]=2.7, \mathrm{p}=0.1075$; Figure 2). For the significant effects, post hoc Tukey multiple comparisons tests revealed a significant difference between stride time (Figure 1) at T0 vs. T2 ( $\mathrm{p}=0.0315)$ but no significant difference between T0 and T1 ( $\mathrm{p}=0.1045)$ or T1 and T2 $(\mathrm{p}=0.4305)$. For stride length, no significant post hoc Tukey tests were found $(\mathrm{p}=0.2779$, $\mathrm{p}=0.0751$ and $\mathrm{p}=0.0961$ for $\mathrm{T} 0$ vs. T1, T0 vs. T2 and T1 vs. T2, respectively; Figure 1 ).

No significant effects of time were found for variability in stride time $(F[1.6,18]=0.75$, $\mathrm{p}=0.4573)$, stride length $(\mathrm{F}[1.2,13]=0.79, \mathrm{p}=0.4136)$, step width $(\mathrm{F}[1.5,17]=3.3, \mathrm{p}=0.0742)$, double support time $(\mathrm{F}[1.8,20]=1.1, \mathrm{p}=0.3606)$ (Figure 1$)$, AP $\operatorname{MoS}(\mathrm{F}[1.8,20]=0.14, \mathrm{p}=0.8522)$ or $\operatorname{ML~MoS~}(\mathrm{F}[1.3,15]=0.8, \mathrm{p}=0.4206)$ (Figure 2). As two parameters showed significant improvements over time, the $\mathrm{T} 2$ values of stride time and length were statistically compared to the healthy control data using independent t-tests, which revealed no significant differences for stride time $(\mathrm{p}=0.397)$ and stride length $(\mathrm{p}=0.550)$.

Regarding the mean self-selected walking speed from the 90 seconds period, there was a statistically significant effect of time on walking speed $(F[1.6,18]=8.1, p=0.0046$; medians of $1.095 \mathrm{~m} / \mathrm{s}, 1.250 \mathrm{~m} / \mathrm{s}$ and $1.260 \mathrm{~m} / \mathrm{s}$ for T0, T1 and T2, respectively; Figure 3), with post hoc Tukey tests revealing significantly faster walking speeds at T2 $(\mathrm{p}=0.018)$ and $\mathrm{T} 1(\mathrm{p}=0.041)$ compared to T0, but not between T1 and T2 ( $\mathrm{p}=0.409)$. Compared to baseline, patients showed significantly decreased VAS-pain scores six weeks and six months after fracture indicating less pain (VAS pain $5.17+1.64,1.83+1.47$ and $1.83+1.53$ respectively; $\mathrm{p}<0.05$ ). Remarkably, four patients with disabling pain after six months of recovery showed no or little improvement in gait and stability over time, indicating that pain plays an important role in the recovery of gait after suffering an OVCF. 

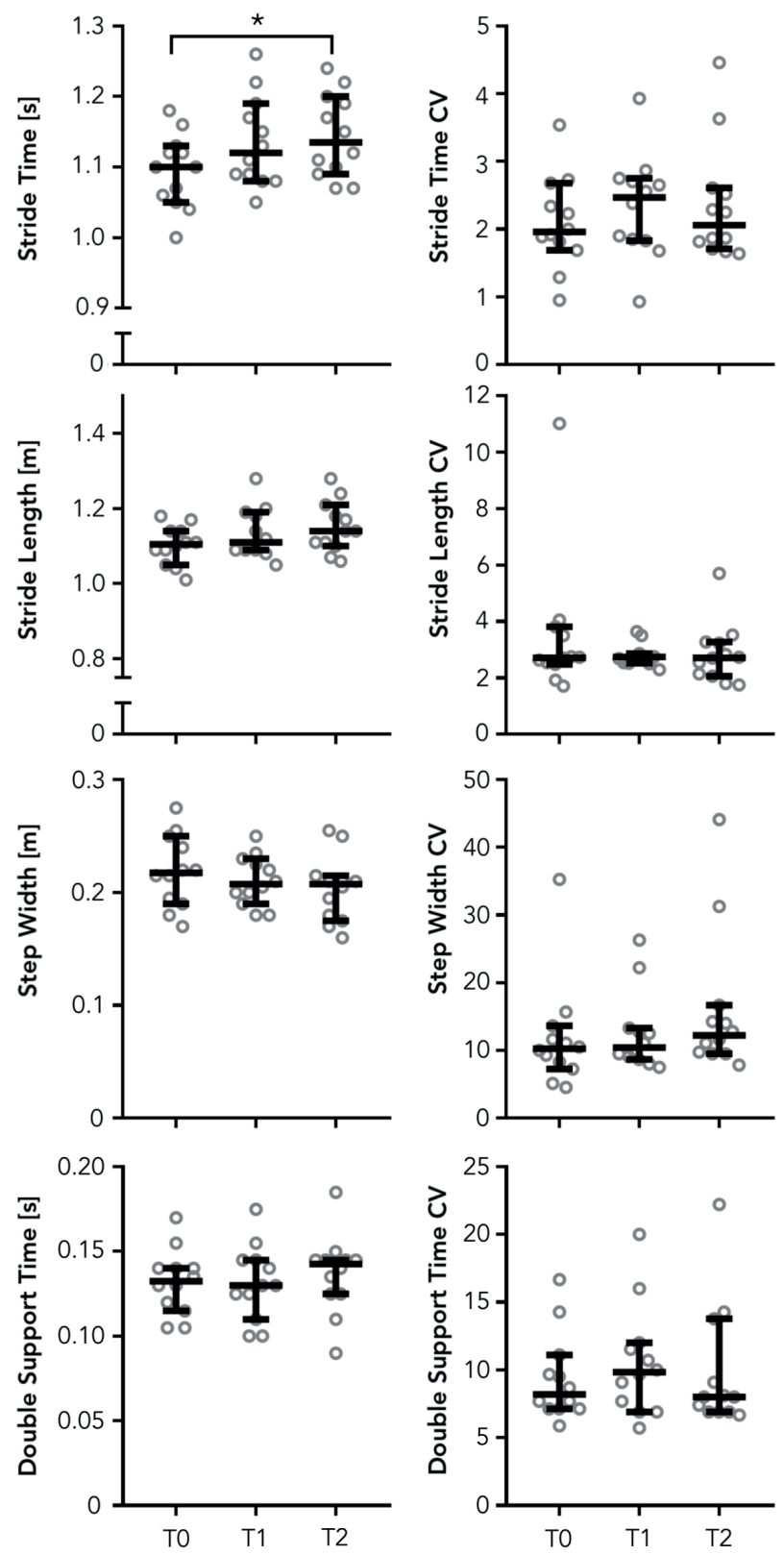

Figure 1. Stride time, stride length, step width and double support time in patients with an OVCF at baseline (T0), after six weeks (T1) and after six months (T2).

Repeated measurements ANOVA demonstrated a significant improvement over time for stride time and length. There was no significant improvement in step width and double support time over time. Data presented for each patient, horizontal bar represents median with 95\% confidence intervals. 

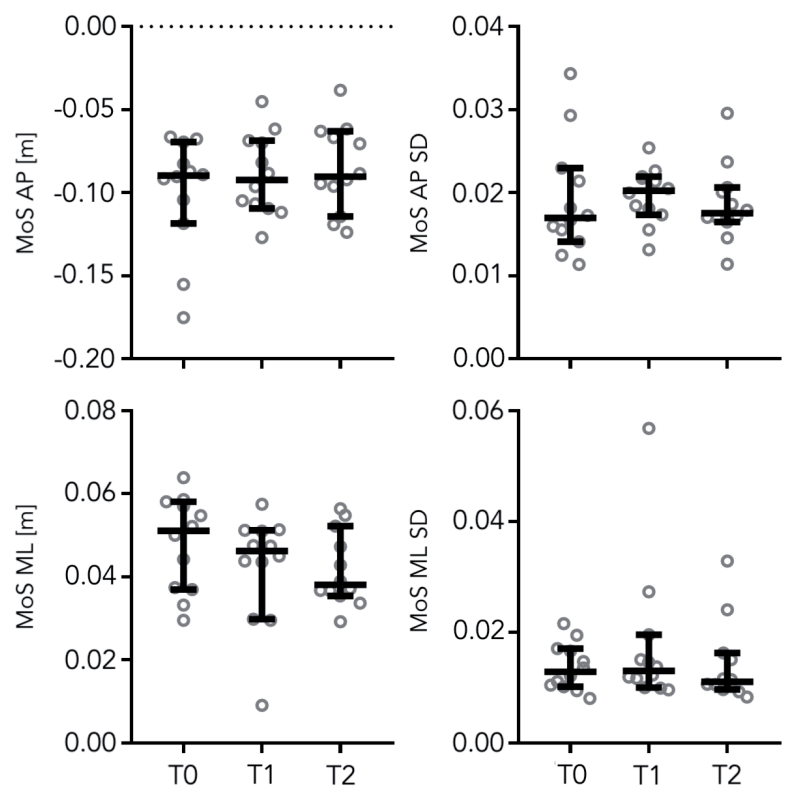

Figure 2. Margins of stability in the anteroposterior and mediolateral direction in patients with an OVCF at baseline (T0), after six weeks (T1) and after six months (T2).

Repeated measurements ANOVA demonstrated no significant differences over time. Data presented for each patient, horizontal bar represents median with $95 \%$ confidence intervals.

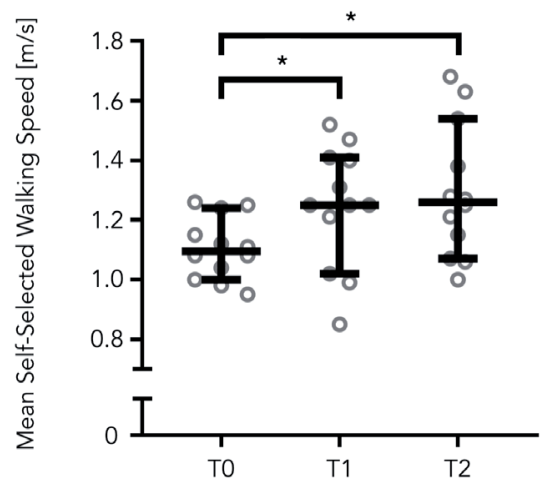

Figure 3. Self-selected walking speed in patients with an OVCF at baseline (T0), after six weeks (T1) and after six months (T2).

There was a significant improvement over time. Data presented for each patient, horizontal bar represents median with $95 \%$ confidence intervals. 


\section{DISCUSSION}

The current study primarily aimed to determine differences in spatiotemporal gait parameters and their variability between patients with an OVCF and healthy age-, gender-, height- and weight-matched participants during treadmill walking. The second aim was to assess whether those parameters and their variability changed over six months following the fracture. In contrast to our first hypothesis, the patients suffering an OVCF did not demonstrate more variable gait compared to the healthy controls. However, patients with an OVCF did walk with shorter, faster and wider strides at the given speed of $1 \mathrm{~m} / \mathrm{s}$ compared to the healthy controls. These differences in stride length and time were no longer present six months after the fracture, indicating a return to a "healthy" stride time and length. Walking speed also improved after six months of conservative treatment. However, step width and both AP and ML margins of stability did not recover over time.

This is the first study in literature to elucidate the spatiotemporal gait characteristics and their variability of older adults suffering an OVCF. Hence comparison with previous studies is limited. In the context of this investigation, patients suffering an OVCF demonstrated shorter, faster and wider strides than healthy age matched controls. These differences are also reported between young and older healthy adults, which may represent limitations in the ability to produce equivalent step lengths due to differences in muscle strength or physical capacity [18]. However, in the case of the current study, it might be speculated that the difference found in spatiotemporal gait parameters at baseline between the OVCF group and healthy controls might be due to pain. The differences in stride time and length at $\mathrm{T} 0$ and the lack of differences at $\mathrm{T} 2$ coincide with the healing of the fracture and with a significant decrease in pain of the patients. Given that those four patients who still had disabling pain at six months, also showed a lack of improvement in the gait parameters, suggests that an association exists between pain and (recovery of) gait after suffering an OVCF. Venmans et al. [19] found that $60 \%$ of conservatively treated patients with acute OVCFs had sufficient pain relief and good functional recovery within approximately three months after the acute fracture. However, $40 \%$ of patients still had disabling pain after one year [19, 20]. Especially these patients should be screened for a history of falls or balance impairment and should follow appropriate (preventative) balance or gait training.

In a study by Hausdorff [16], stride time CV appeared to be a useful parameter to assess the risk of falling in elderly. In the current study, no significant difference in stride time CV was found between the OVCF group and healthy controls. This is in accordance with the study of de Groot et al. [8], in which only patients with a flexed posture demonstrated a greater variability in stride time in comparison to patients with a normal posture whereas there were no differences between the groups in the presence of vertebral fractures. However, although there was no significant difference in stride time CV, in the 
current study vertebral fractures did seem to lead to alterations in AP and ML MoS, both with relatively greater stability than the control participants (when accounting for the marker set differences). The increased ML MoS corresponds with the significantly wider steps observed in the OVCF group. A wide base of support during gait is typically indicative of poor or cautious dynamic balance control [21]. However, the shorter stride length but slightly increased AP MoS (when accounting for marker set differences) implies that it was not the change in base of support that affected the MoS (as the value would then be smaller in the patients), but rather that the centre of mass position and/or accelerations that were less anterior in the OVCF group. This may represent a compensation for pain or discomfort to reduce fluctuations in the upper body. The likelihood that the AP MoS difference is mainly due to the centre of mass characteristics is supported by the longitudinal data, as despite an increased stride length over time (and therefore a larger base of support) these changes are not reflected in the AP MoS.

Gait is an important indicator of health and can be influenced by many variables [16, 22-24]. Older adults with chronic low back pain exhibit significantly different gait patterns compared to age- and sex-matched adults without chronic low back pain [25]. In the current study it was demonstrated that only two of all measured gait parameters improved and returned to "healthy" levels after six months of conservative treatment (self-selected gait speed and stride time at $1 \mathrm{~m} / \mathrm{s}$ ). Most parameters, including step width, double support time and MoS, remained unchanged (Figure 1). These parameters are closely related to gait stability. Therefore, it appears that overall, the capacity of the patients' gait in terms of stride length and speed improved over time, but deficits in stability did not. This may imply that the OVCF population are at a greater risk for incident disability, such as falling, than their healthy counterparts even six months after conservative treatment [24]. These findings suggest that an OVCF can be the serious beginning of a downward cascade which should be taken into account in the multimodal treatment of vertebral fractures and osteoporosis.

An important limitation of this study is the small number of subjects that were included, which may have affected the power of the study. However, as patients suffering an OVCF are often unable to participate due to incapacitating pain and inability to walk independently, together with the fact that these patients are typically characterized by a combination of physiological, psychological and social problems, gait and stability studies on this specific condition will always be faced with this challenge. However, the individual data points and the changes over time do appear to confirm the statistical outcomes and so we believe that this may not have affected the overall conclusions of the study. A second limitation is the lack of consistent marker sets in the patient and control groups. However, the pilot study with 360 data points in the Bland-Altman plots addressed this limitation providing sufficient data to estimate the absolute difference in the marker sets. Furthermore, the longitudinal analysis has not been affected by this limitation as the patients were measured every time with the same marker set. 
In conclusion, the results of the current study indicated that patients suffering from an OVCF walk with shorter, faster and wider strides as compared to their healthy counterparts and adopt a less stable body configuration in the anterior direction, potentially increasing their risk of forward falls if perturbed. Although stride time and stride length improve over time even reaching healthy levels again, patients significantly deviate from normal gait patterns (e.g. in stability and step width) after six months of conservative treatment. 


\section{REFERENCES}

1. Johnell O, Kanis JA. An estimate of the worldwide prevalence and disability associated with osteoporotic fractures. Osteoporos Int 2006;17(12):1726-33 doi: 10.1007/s00198-006-0172-4.

2. Luthman S, Widen J, Borgstrom F. Appropriateness criteria for treatment of osteoporotic vertebral compression fractures. Osteoporos Int 2018;29(4):793-804 doi: 10.1007/s00198-0174348-x.

3. Ismail AA, Cooper C, Felsenberg D, et al. Number and type of vertebral deformities: epidemiological characteristics and relation to back pain and height loss. European Vertebral Osteoporosis Study Group. Osteoporos Int 1999;9(3):206-13

4. Lindsay R, Silverman SL, Cooper C, et al. Risk of new vertebral fracture in the year following a fracture. JAMA 2001;285(3):320-3

5. Ailon T, Shaffrey CI, Lenke LG, Harrop JS, Smith JS. Progressive Spinal Kyphosis in the Aging Population. Neurosurgery 2015;77 Suppl 4:S164-72 doi: 10.1227/NEU.00000000000000944.

6. Glassman SD, Bridwell K, Dimar JR, Horton W, Berven S, Schwab F. The impact of positive sagittal balance in adult spinal deformity. Spine (Phila Pa 1976) 2005;30(18):2024-9

7. Lee HM, Park SY, Lee SH, Suh SW, Hong JY. Comparative analysis of clinical outcomes in patients with osteoporotic vertebral compression fractures (OVCFs): conservative treatment versus balloon kyphoplasty. Spine J 2012;12(11):998-1005 doi: 10.1016/j.spinee.2012.08.024.

8. de Groot MH, van der Jagt-Willems HC, van Campen JP, Lems WF, Beijnen JH, Lamoth CJ. A flexed posture in elderly patients is associated with impairments in postural control during walking. Gait Posture 2014;39(2):767-72 doi: 10.1016/j.gaitpost.2013.10.015.

9. van der Jagt-Willems HC, de Groot MH, van Campen JP, Lamoth CJ, Lems WF. Associations between vertebral fractures, increased thoracic kyphosis, a flexed posture and falls in older adults: a prospective cohort study. BMC Geriatr 2015;15:34 doi: 10.1186/s12877-015-0018-z.

10. Talbot LA, Musiol RJ, Witham EK, Metter EJ. Falls in young, middle-aged and older community dwelling adults: perceived cause, environmental factors and injury. BMC Public Health 2005;5:86 doi: 10.1186/1471-2458-5-86.

11. Hof AL, Gazendam MG, Sinke WE. The condition for dynamic stability. J Biomech 2005;38(1):18 doi: 10.1016/j.jbiomech.2004.03.025.

12. McCrum C, Eysel-Gosepath K, Epro G, et al. Associations Between Bipedal Stance Stability and Locomotor Stability Following a Trip in Unilateral Vestibulopathy. J Appl Biomech 2017;33(2):112-17 doi: 10.1123/jab.2016-0004.

13. Mackey DC, Robinovitch SN. Postural steadiness during quiet stance does not associate with ability to recover balance in older women. Clin Biomech (Bristol, Avon) 2005;20(8):776-83 doi: 10.1016/j.clinbiomech.2005.05.002.

14. Owings TM, Pavol MJ, Foley KT, Grabiner MD. Measures of postural stability are not predictors of recovery from large postural disturbances in healthy older adults. J Am Geriatr Soc 2000;48(1):42-50

15. Yang F, Pai YC. Adaptive control of center of mass (global) motion and its joint (local) origin in gait. J Biomech 2014;47(11):2797-800 doi: 10.1016/j.jbiomech.2014.06.001.

16. Hausdorff JM. Gait variability: methods, modeling and meaning. J Neuroeng Rehabil 2005;2:19 doi: 10.1186/1743-0003-2-19.

17. Zeni JA, Jr., Higginson JS. Gait parameters and stride-to-stride variability during familiarization to walking on a split-belt treadmill. Clin Biomech (Bristol, Avon) 2010;25(4):383-6 doi: 10.1016/j.clinbiomech.2009.11.002. 
18. Hafer JF, Boyer KA. Age related differences in segment coordination and its variability during gait. Gait Posture 2018;62:92-98 doi: 10.1016/j.gaitpost.2018.02.021.

19. Venmans A, Klazen CA, Lohle PN, Mali WP, van Rooij WJ. Natural history of pain in patients with conservatively treated osteoporotic vertebral compression fractures: results from VERTOS II. AJNR Am J Neuroradiol 2012;33(3):519-21 doi: 10.3174/ajnr.A2817.

20. Muratore M, Ferrera A, Masse A, Bistolfi A. Osteoporotic vertebral fractures: predictive factors for conservative treatment failure. A systematic review. Eur Spine J 2018;27(10):2565-76 doi: 10.1007/s00586-017-5340-z.

21. Gabell A, Nayak US. The effect of age on variability in gait. J Gerontol 1984;39(6):662-6

22. Verghese J, Holtzer R, Lipton RB, Wang C. Quantitative gait markers and incident fall risk in older adults. J Gerontol A Biol Sci Med Sci 2009;64(8):896-901 doi: 10.1093/gerona/glp033.

23. de Kruijf M, Verlinden VJ, Huygen FJ, et al. Chronic joint pain in the lower body is associated with gait differences independent from radiographic osteoarthritis. Gait Posture 2015;42(3):354-9 doi: 10.1016/j.gaitpost.2015.06.193.

24. Abellan van Kan G, Rolland Y, Andrieu S, et al. Gait speed at usual pace as a predictor of adverse outcomes in community-dwelling older people an International Academy on Nutrition and Aging (IANA) Task Force. J Nutr Health Aging 2009;13(10):881-9

25. Hicks GE, Sions JM, Coyle PC, Pohlig RT. Altered spatiotemporal characteristics of gait in older adults with chronic low back pain. Gait Posture 2017;55:172-76 doi: 10.1016/j.gaitpost.2017.04.027. 


\section{SUPPLEMENTARY INFORMATION}

\section{Marker Set Comparison Pilot}

\section{Rationale}

In order to determine the differences between groups in the main study that could simply be due to absolute differences in the two different reduced marker sets, this pilot study conducted measurements of participants walking while wearing both marker sets. In this way, we could estimate the absolute difference in the parameters of interest due to the marker sets and determine if the differences found in the main study exceeded these.

\section{Methods}

Six healthy young adults (age [mean $\pm S D$ ]: $23.3 \pm 3.7$ years; height: $175.6 \pm 6.4 \mathrm{~cm}$; weight: $66.5 \pm 9.1 \mathrm{~kg}$ ) participated in the pilot measurements. These measurements were registered with the medical ethics committee of Maastricht University (number: 2018-0568; METC azM/UM), written informed consent was obtained from participants and the measurements were conducted in accordance with the Declaration of Helsinki.

Sixteen retroreflective markers were attached to anatomical landmarks of the participants comprising both marker sets used in the main study (see main text for details). Participants walked for three minutes at $1 \mathrm{~m} / \mathrm{s}$ on the Computer Assisted Rehabilitation Environment Extended (CAREN; Motekforce Link, Amsterdam, The Netherlands), as in the main study. In post-processing, copies of the motion capture data were made and marker coordinates were removed for each of the marker sets respectively so that one file contained only the six marker set and the other contained only the ten marker set for the same motion capture recording. Following this, the final 60 strides from each recording were analyzed for the gait parameters stride time, stride length and double support time, with the final 60 steps analyzed for step width. Additionally, the margins of stability (MoS) were calculated in both the anteroposterior (AP) and mediolateral (ML) directions at foot touchdown for the final 60 steps as the AP or ML distance between the boundary of the base of support (BoS; the hallux marker) and the extrapolated centre of mass (XCoM), as defined by Hof et al. (2005), adapted for the reduced kinematic model based on Süptitz et al. (2013), whereby the position of the centre of mass was the average of the trochantor makers and the velocity of the centre of mass was estimated as:

$$
0.5\left(\frac{\mathrm{V}_{\text {Trol }}+\mathrm{V}_{\text {Trol }}}{2}+\mathrm{V}_{\mathrm{c} 7}\right)
$$

where $\mathrm{V}_{\text {Trol }}, \mathrm{V}_{\text {TroR }}$ and $\mathrm{V}_{\mathrm{c} 7}$ are the anteroposterior or mediolateral velocities of the trochanter and $\mathrm{C} 7$ markers respectively. For the AP direction, the velocity of the treadmill belt was added. 

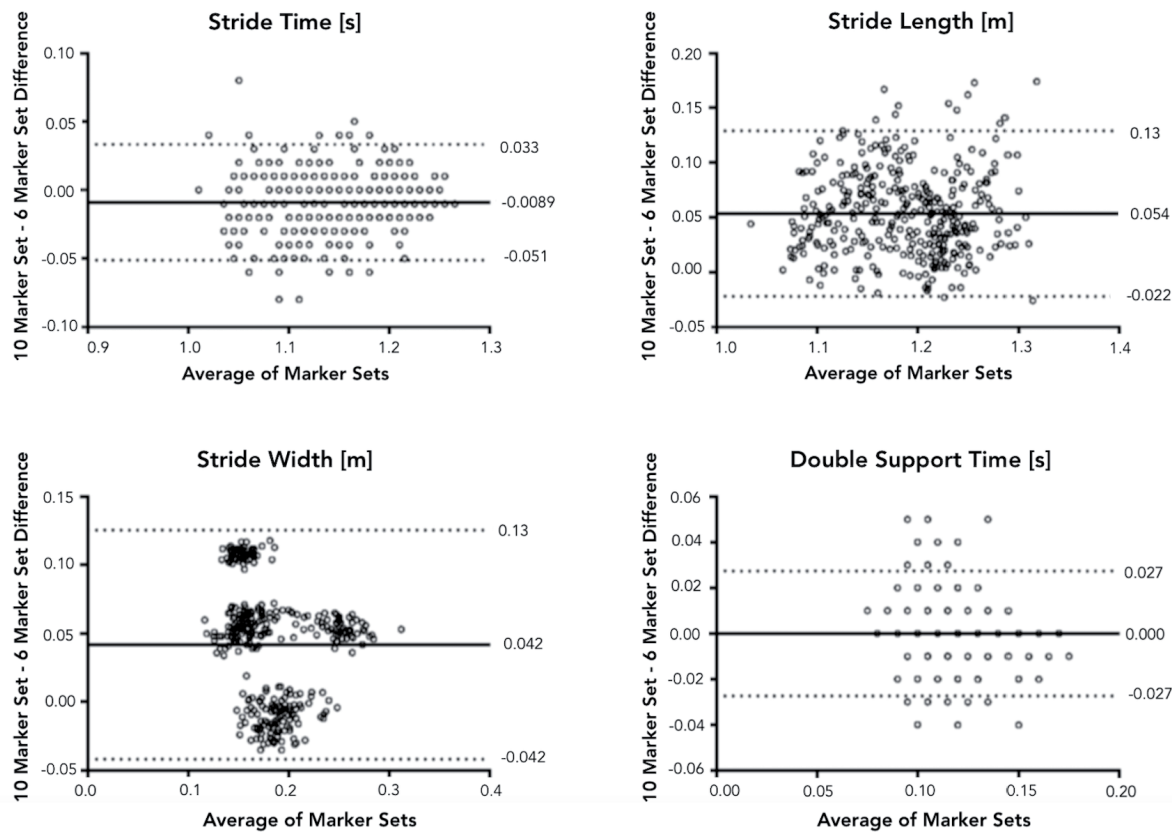

Supplementary Figure 1. Bland-Altman plots of the spatiotemporal parameters stride time, stride length, step width and double support time from 60 strides (or steps for step width) from six young healthy participants (total trials $\mathrm{n}=360$ ). Mean bias is indicated by the solid black line and upper and lower $95 \%$ limits of agreement are indicated by the dotted lines.
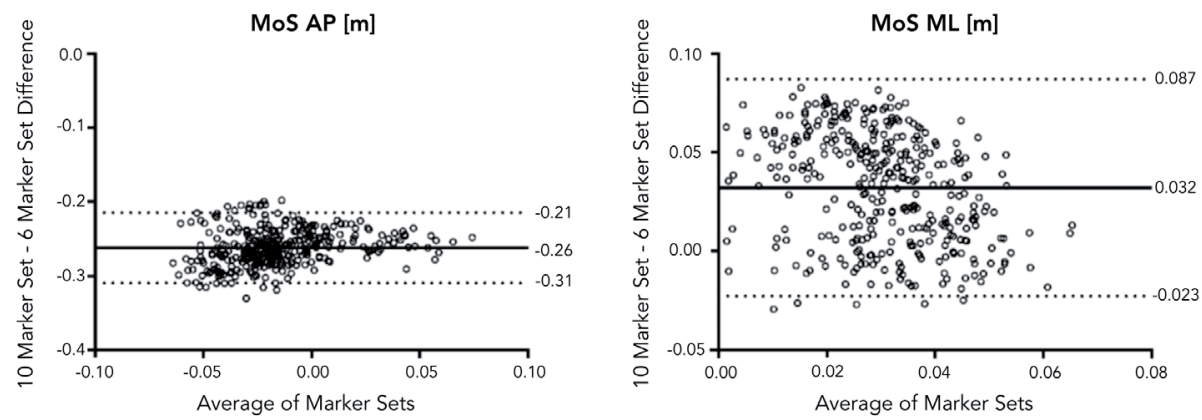

Supplementary Figure 2. Bland-Altman plots of the margins of stability in the anteroposterior direction (MoS AP) and the mediolateral direction (MoS ML) from 60 steps of six young healthy participants (total trials $\mathrm{n}=360$ ). Mean bias is indicated by the solid black line and upper and lower $95 \%$ limits of agreement are indicated by the dotted lines. 




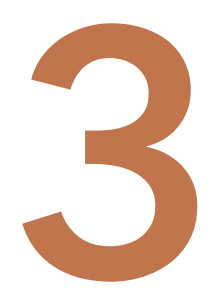

The effect of a semirigid thoracolumbar orthosis on gait and sagittal alignment in patients with an osteoporotic vertebral compression fracture

Jacobs E, Senden R, McCrum C,

van Rhijn LW, Meijer K, Willems PC

Clin Interv Aging. 2019 Apr 11;14:671-680 


\section{ABSTRACT}

Background - An important goal in the treatment of osteoporotic vertebral compression fractures (OVCFs) is the prevention of new vertebral fractures and the subsequent progression to global sagittal malalignment. Current conservative treatment is multimodal and comprises analgesics, medication for osteoporosis and physical therapy. However, little is known about the value of orthoses in the treatment of OVCFs.

Aims - The primary purpose of this study was to examine the direct effect of a semirigid thoracolumbar orthosis on gait in patients suffering from an OVCF. The secondary purpose was to evaluate changes in gait, radiographic sagittal alignment, pain and quality of life over time.

Methods - Fifteen postmenopausal patients with an OVCF were treated with a semirigid thoracolumbar orthosis. At baseline, after six weeks and after six months gait analysis was performed with a dual belt instrumented treadmill with a 180 degrees projection screen providing a virtual environment (Computer Assisted Rehabilitation Environment) combined with clinical and radiographic assessments.

Results - At baseline, bracing caused a significantly more upright posture during walking and patients walked faster, with larger strides, longer stride times and lower cadence compared to walking without orthosis. After six weeks, radiographic and dynamic sagittal alignment improved compared to baseline. The observed effect was gone after six months when the orthosis was not worn anymore.

Conclusion - A semirigid thoracolumbar orthosis seems to have a positive effect on gait and stability in patients suffering an OVCF as was shown by a more upright posture, which may result in decreased compressive loading of the vertebrae. For studying the true effectiveness of dynamic bracing in the treatment of OVCFs, a prospective, randomized controlled trial will be needed. 


\section{INTRODUCTION}

Osteoporosis is characterized by a low bone mineral density and a resulting propensity to fractures, of which vertebral compression fractures are the most common clinical manifestation. In our ageing society the impact of osteoporotic vertebral compression fractures (OVCFs) on medical and economic costs is rapidly increasing [1].

Postmenopausal women who present with an initial vertebral compression fracture are at substantial risk for a subsequent vertebral fracture within one year [2]. When fractured, the vertebra is commonly deformed by disproportionate height loss from the anterior vertebral body resulting in wedging [3]. Wedge accumulation over multiple thoracolumbar levels may lead to subsequent spinal deformity [4]. Inability to compensate for the alternating load distribution of the spine leads to global spinal sagittal malalignment [4]. Increased anterior spinal loading has been associated with a downward spiral of subsequent vertebral compression fractures [5].

In thoracolumbar hyperkyphosis there is an increased anterior bending moment on the trunk which shifts the body's centre of mass forward, i.e., closer to the anterior boundary of the base of support under the feet. It has been shown that global sagittal malalignment causes impaired postural control, slower gait and a wider base of support with stance and gait $[3,6]$. Impaired postural control during walking is a major risk factor for falls [7]. Critically, falls among older adults who are frail and have osteoporosis are associated with high morbidity and mortality and may need high-cost medical interventions $[1,8]$.

Treatment of OVCFs should aim to break the downward spiral of recurrent vertebral compression fractures and to prevent the subsequent progression of hyperkyphosis and resultant global sagittal malalignment. Furthermore, it should intend to prevent or slowdown the decline in postural control, while limiting the increasing risk of falling in these patients [7, 9]. Current conservative management of symptomatic OVCFs is multimodal and comprises analgesics, medication for osteoporosis, physical therapy, and bracing $[10,11]$. The use of conventional, rigid spinal orthoses is limited in patients suffering from osteoporosis due to the suspected subsequent atrophy of the trunk muscles and restricted respiration leading to low compliance [12, 13]. In order to address these drawbacks, the concept of "dynamic bracing" has been introduced using semirigid thoracolumbar orthoses in which trunk muscle strength is improved and compliance is increased [12-15]. These orthoses are commonly used to treat traumatic vertebral fractures, but there is little knowledge on their use for OVCFs [10, 11, 13, 16]. The primary goal of dynamic bracing in the conservative treatment of OVCFs is to reduce pain by stabilising the spine and allowing for muscle spasm relief [13]. Another goal is to maintain neutral spinal alignment and to limit flexion, thus reducing anterior axial loading on the fractured vertebrae [14]. However, the actual effect of dynamic bracing on the sagittal alignment of the spine in patients suffering from an OVCF has only been 
determined by quantification of the regional kyphosis angle on static radiographs [12, 13]. No studies are available evaluating the effect of a dynamic orthosis on gait and global sagittal alignment of the spine $[12,14,15]$. The data in previous studies regarding balance is mainly retrieved during static tasks such as standing, but it is important to note that stability control during static tasks shows only little relationship with more dynamic tasks as performed during activities of daily living [7, 8, 17].

The primary aim of this study was to examine the direct effect of a semirigid thoracolumbar orthosis in patients suffering from an OVCF on spatiotemporal gait parameters, trunk motion and stance-phase knee flexion while walking. The secondary study purpose was to evaluate changes over time in gait, radiographical sagittal alignment of the spine, and pain and quality of life in these patients.

\section{METHODS}

\subsection{Participants}

This was an observational, single-centre study approved by the Maastricht University Medical Centre institutional review board (NL52978.057.15). The study was explained before obtaining patient written informed consent and conducted in accordance with the Declaration of Helsinki. Inclusion of participants was conducted from September 2015 to March 2018. Fifteen, consecutive, adult postmenopausal patients presenting at the Emergency Department suffering from a symptomatic, new OVCF of the thoracolumbar spine were enrolled. Inclusion criteria were female gender, age 55 years or older, symptomatic OVCF (less than 3 weeks old, visible on thoracolumbar radiographs) and ambulatory. Exclusion criteria were unstable fractures, previous OVCFs, not fully ambulatory, the need of walking aids, active cancer, and body weight exceeding the equipment rating for the treadmill (135 kg). Demographic data included patient age, body height, body weight and Charlson Comorbidity Index. Informed consent was obtained from all individual participants included in the study. Data were obtained at baseline (T0), at six weeks (T1) and at six months after baseline (T2). Since this study was set up as an exploratory study, no power calculation was performed for the desired primary outcome measure.

\subsection{Treatment}

All subjects were prescribed to wear the thoracolumbar orthosis Osteolind ${ }^{\circledast}$ Plus (Werkmeister, Wanfried, Germany) in the first six weeks for the entire day and optionally during the night. The orthosis is semirigid and consists of a malleable metal frame with soft padding and a system of belts with fleece. The orthoses were adjusted for each patient by an orthopaedic technician. After the six weeks visit, patients were requested to wear the orthosis at least six hours daily, and after three months for at least three hours daily until the final follow-up visit at six months. The actual time patients wore 
the orthosis per day was reported in a patient diary during the entire study period. The diary was also used to monitor comfort and complications.

Patients were referred to the rheumatology department for treatment of osteoporosis. Analgesic medication was prescribed whereas physical therapy was not.

\subsection{Gait and Stability Analysis}

Gait measurements were performed in a Computer Assisted Rehabilitation Environment (CAREN; Motekforce Link, Amsterdam, The Netherlands) by two certified clinical operators. The CAREN system includes a dual-belt treadmill with two instrumented force plates $(1000 \mathrm{~Hz})$, a 12 -camera motion capture system (100Hz; Vicon Motion Systems, Oxford, UK) and a virtual environment that provided optic flow on a $180^{\circ}$ semi-cylindrical screen. Participants wore a safety harness connected to an overhead frame. Ten retroreflective markers were attached to anatomical landmarks (two acromion markers, four pelvic markers, two lateral distal femur markers and two lateral malleolus markers) and were tracked by the motion capture system [18]. The marker tracks were filtered using a low pass second order Butterworth filter (zero-phase) with a cut-off frequency of $12 \mathrm{~Hz}$. Foot touchdown and toe-off were determined using the treadmill force plates (50N threshold) in combination with a marker-based method [12]. This combined method was used to be able to accurately account for foot touchdowns and toe-offs occurring in the centre of the treadmill triggering both force plates simultaneously. For all steps, the foot marker method was used and then corrected based on the average discrepancy between the force plate method and the marker method timing for all steps that contacted only one force plate.

Participants first completed a familiarization trial consisting of 90 seconds walking at $1 \mathrm{~m} / \mathrm{s}$ followed by 90 seconds walking at self-paced speed. Following familiarization, patients walked under two conditions: firstly, without wearing the orthosis and subsequently while wearing the orthosis. During each condition, patients first walked at self-paced mode for 90 seconds and subsequently at a fixed speed of $1 \mathrm{~m} / \mathrm{s}$ for 90 seconds.

Collected gait data were analyzed in Matlab (version 9.4, R2016a) using previously published algorithms $[19,20]$. The following spatiotemporal parameters were calculated: Walking speed defined as the average treadmill speed measured during self-paced mode; Step frequency defined as the inverse of the average duration between two subsequent heel-strikes; Step width calculated as the ML-distance between the ankle markers at the moment of heel-contact and step length defined as the AP-distance between these markers at the moment of heel-contact. Stability was expressed using margins of stability $(\mathrm{MoS})$, calculated in both the anteroposterior (AP) and mediolateral (ML) directions at foot touchdown as the AP or ML distance between the boundary of the base of support (BoS; the ankle marker) and the extrapolated centre of mass $\left(\mathrm{X}_{\mathrm{CoM}}\right)$ in the corresponding plane, as defined by Hof et al. [21]. For the estimation of the centre of mass position and velocity, the average positions of the four pelvis markers were used. Stance-phase knee 
flexion was determined as the knee flexion measured during terminal stance. Trunk motion, defined as the position of the trunk with regards to the pelvis, was calculated as the AP distance between the average of both acromion markers and the middle of the pelvis markers. The average distance over a gait cycle was calculated. Positive values indicate forward positioning of the trunk (positive sagittal alignment) whereas negative values indicate backward leaning. Produced gait and stability parameters were based on all recorded steps. For analysis the averages of all these parameters were used.

\subsection{Radiographic Assessment}

Static sagittal alignment was analyzed on standardized lateral full-spine radiographs using validated software (Surgimap, Nemaris Inc., New York, NY). Pelvic parameters measured were Pelvic Incidence (PI), Pelvic Tilt (PT), and Sacral Slope (SS). Regional spinal parameters included Lumbar Lordosis (LL, L1-S1) and Thoracic Kyphosis (TK, T4-T12). Sagittal alignment was assessed by Sagittal Vertical Axis (SVA), Global Sagittal Alignment (GSA) and Global Alignment and Proportion score (GAP score) [22, 23]. The wedge angle of each fractured vertebra was measured (the angle formed between the two lines drawn parallel to the superior and inferior endplates of the fractured vertebra). All patients had bone mineral density measurements (BMD) of lumbar spine using a dual-energy-X-ray absorptiometry device. The World Health Organization (WHO) classification was used to define osteoporosis as T-score $\leq-2.5$ (or osteopenia as T-score $\leq 1.0$ ) at the lumbar spine.

\subsection{Quality of Life Assessment}

Pain was assessed using a Visual Analog Scale (VAS), ranging from 0 to 10, where level 10 implies extreme pain and level 0 no pain at all. Quality of life was assessed using the Quality of Life Questionnaire of the European Foundation for Osteoporosis (QUALEFFO 41).

\subsection{Statistics}

Data was analyzed using SPSS Version 25 for Windows (SPSS, Inc. Chicago, IL). To investigate the effect of bracing on gait, paired t-tests were performed. Linear mixed effect model analysis (LSD correction) was performed to test for differences in gait, stability, trunk motion, radiographic sagittal alignment, pain and quality of life between the various time points. A significance level of 0.05 was used.

\section{RESULTS}

Fifteen female participants with a symptomatic OVCF were included [mean age 69 (55-78) yrs, mean weight $69 \pm 11 \mathrm{~kg}$, mean height $1.61 \pm 0.06 \mathrm{~m}$, mean T-score $-1.65 \pm 1.41$ for the lumbar spine; Table 1]. For the secondary purpose, the six weeks and six months data 
Chapter 3

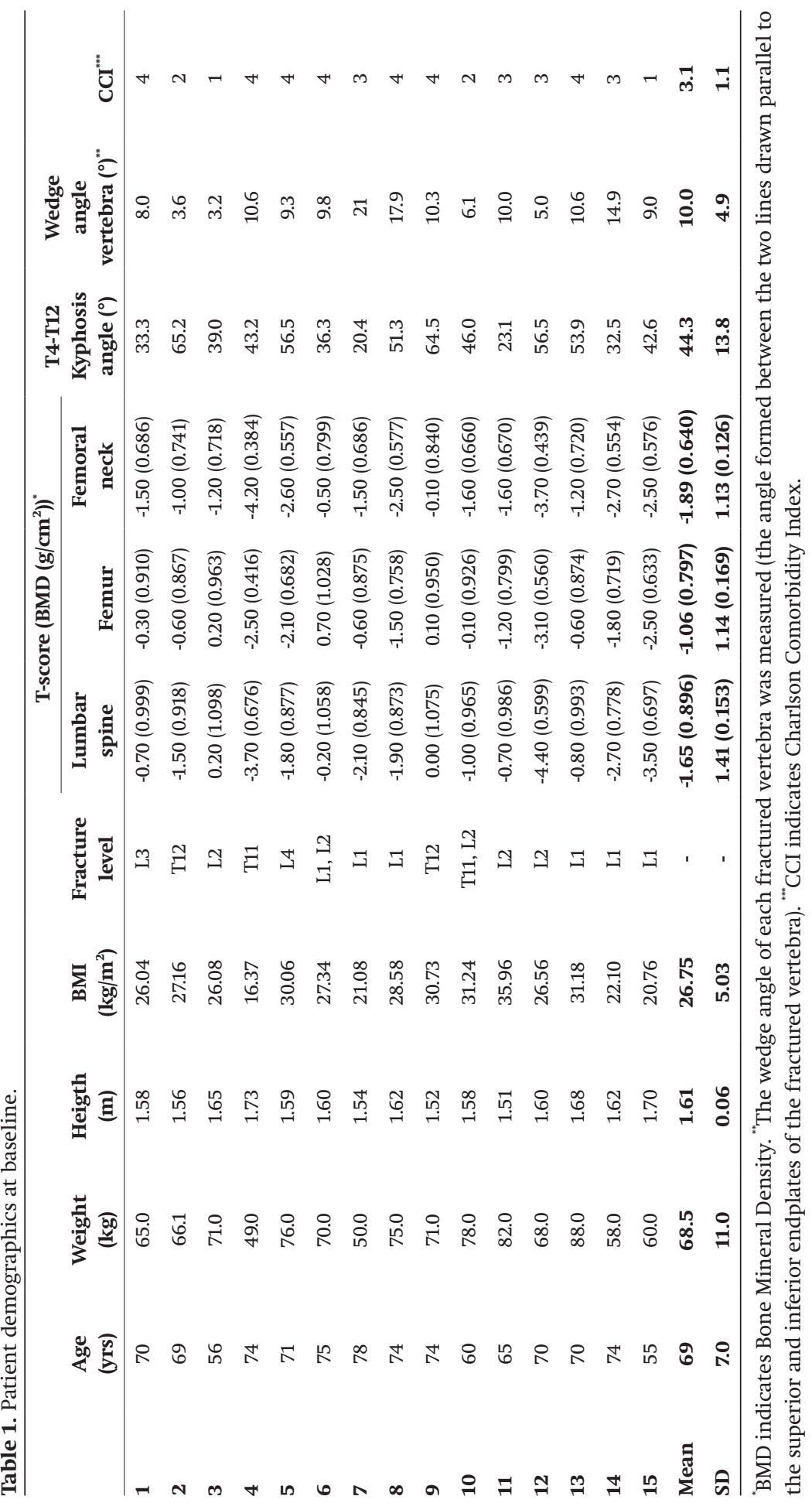


were available of eleven patients since four patients were not able or not willing to conduct follow-up measurements for various reasons. All patients wore the orthosis the entire day for the first six weeks; however, after the six weeks visit the time wearing the orthosis varied considerably among the participants (1 to 12 hours per day). Patients did not report any discomfort or complications wearing the orthosis.

\subsection{Effect of Bracing}

At baseline, walking with the orthosis resulted in a statistically significant decreased trunk motion as compared to walking without the orthosis (51\% decline, $\mathrm{p}=0.006)$. In addition, while wearing the orthosis patients tended to walk faster, with larger strides, longer stride times and lower cadence (Table 2). Stance-phase knee flexion decreased non-significantly when walking with orthosis, however large variation existed between patients as indicated by large standard deviations. Step width and MoS were comparable Gaiteen both walking conditions (Table 2).

Table 2. Summary of the spatiotemporal and stability parameters, trunk motion and stance-phase knee flexion at baseline (mean $\underline{ \pm}$ standard deviation).

\begin{tabular}{lccc}
\hline Gait & No brace $(\mathbf{n}=\mathbf{1 5})$ & Brace $\mathbf{( n = 1 5 )}$ & P-value \\
\hline Stride length (m) & $1.11 \pm 0.05$ & $1.13 \pm 0.06$ & 0.417 \\
Speed (m/s) & $1.07 \pm 0.12$ & $1.13 \pm 0.17$ & 0.109 \\
Stride time (s) & $1.11 \pm 0.05$ & $1.13 \pm 0.06$ & 0.140 \\
Step width (m) & $0.22 \pm 0.03$ & $0.22 \pm 0.03$ & 0.685 \\
Cadence (st/min) & $108.99 \pm 5.42$ & $106.78 \pm 5.26$ & 0.156 \\
Trunk motion (cm) & $3.60 \pm 2.59$ & $2.38 \pm 2.84$ & 0.006 \\
Knee flexion $\left(^{\circ}\right)$ & $7.37 \pm 6.87$ & $6.76 \pm 6.02$ & 0.305
\end{tabular}

\begin{tabular}{lccc} 
Stability & No brace $(\mathbf{n}=\mathbf{1 5})$ & Brace $(\mathbf{n}=\mathbf{1 5})$ & P-value \\
\hline MoS ML (m) & $0.05 \pm 0.01$ & $0.05 \pm 0.01$ & 0.675 \\
MoS AP (m) & $-0.08 \pm 0.03$ & $-0.07 \pm 0.03$ & 0.882 \\
\hline
\end{tabular}

Indicates a statistically significant difference between walking with and without orthosis $(\mathrm{p}<0.05)$.

Stability after the fracture, spatiotemporal parameters, MoS, stance-phase kP-valuexion and also trunk motion were comparable between the two walking conditions (Figure 1B). Six months after the fracture, there was again a significant reduced trunk motion when walking with orthosis (Figure $1 \mathrm{~B}$ versus $1 \mathrm{C}$ ). The other outcome measures were comparable between the two walking conditions six months after baseline. 

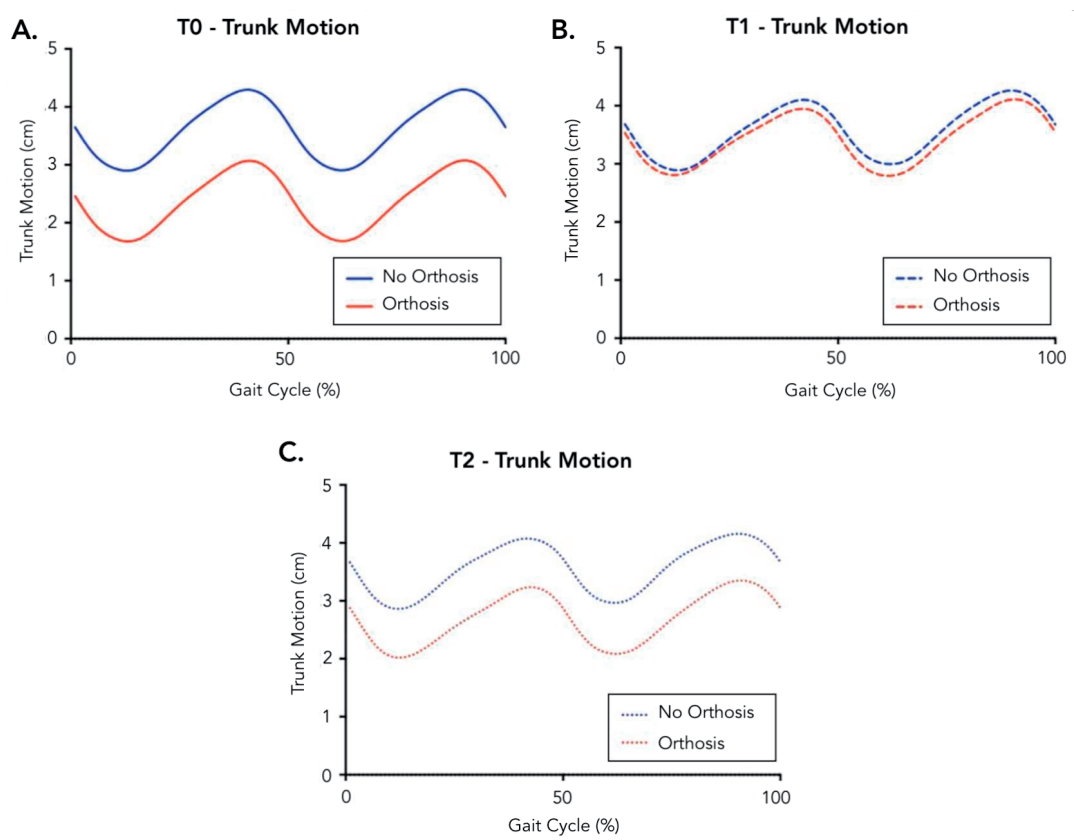

Figure 1. Trunk motion over time. A more positive value indicates a more anterior position of the trunk in comparison to the pelvis. A. Trunk motion at baseline. Significant more flexed posture without orthosis $(\mathrm{p}<0.05)$. B. Trunk motion after six weeks wearing the orthosis shows no difference between walking with and without orthosis. C. Trunk motion after six months, showing a comparable pattern as found at baseline $(\mathrm{p}<0.05)$.

\subsection{Changes over Time}

\subsubsection{Gait and Stability Analysis (Walking without Orthosis).}

Patients showed a gradual improvement over time in trunk motion (decrease of $8 \%$ $(\mathrm{p}=0.660)$ and $21 \%(\mathrm{p}=0.270)$ at six weeks and six months, respectively). Spatiotemporal parameters significantly improved compared to baseline, with faster speed (increase of $14 \%$ at six weeks $(\mathrm{p}=0.004)$ and $19 \%$ at six months $(\mathrm{p}<0.001)$, larger strides $(\mathrm{p}=0.039$ at six weeks $(4 \%), \mathrm{p}=0.007$ at six months ( $5 \%)$ ), longer stride times ( $\mathrm{p}=0.008$ at six weeks $(5 \%)$, $\mathrm{p}=0.003$ at six months $(6 \%)$ ) and lower cadence ( $\mathrm{p}=0.011$ at six weeks $(4 \%), \mathrm{p}=0.003$ at six months (4\%)) (Table 3). Step width decreased over time, but the decrease with regard to baseline was only significant at six months $(\mathrm{p}=0.041)$. Also stability changed over time, showing decreased MoS in the ML direction and increased MoS in the AP direction at SIX weeks ( $\mathrm{p}=0.077$ and $\mathrm{p}=0.148$ respectively)) and at six months ( $\mathrm{p}=0.137$ and $\mathrm{p}=0.045$ ) with regard to baseline (Table 3). Stance-phase knee flexion was comparable between the time points, showing differences smaller than $2^{\circ}$. Changes in gait and stability between six weeks and six months were not significant. 
Table 3. Spatiotemporal and stability parameters, trunk motion and stance-phase knee flexion at the different time points (mean \pm standard deviation).

\begin{tabular}{|c|c|c|c|c|c|c|}
\hline Gait & T0 (n=11) & T1 (n=11) & T2 (n=11) & $\begin{array}{c}\text { T0 vs. T1 } \\
\text { p-value }\end{array}$ & $\begin{array}{c}\text { T0 vs. T2 } \\
\text { p-value }\end{array}$ & $\begin{array}{c}\text { T1 vs. T2 } \\
\text { p-value }\end{array}$ \\
\hline Stride length (m) & $1.10 \pm 0.05$ & $1.14 \pm 0.07$ & $1.15 \pm 0.07$ & $0.039^{*}$ & $0.007^{*}$ & 0.414 \\
\hline Speed (m/s) & $1.09 \pm 0.10$ & $1.24 \pm 0.21$ & $1.30 \pm 0.23$ & $0.004^{\circ}$ & $<0.001^{\circ}$ & 0.252 \\
\hline Stride time (s) & $1.09 \pm 0.05$ & $1.14 \pm 0.07$ & $1.15 \pm 0.06$ & $0.008^{*}$ & $0.003^{*}$ & 0.613 \\
\hline Step width (m) & $0.22 \pm 0.03$ & $0.21 \pm 0.02$ & $0.20 \pm 0.03$ & 0.255 & $0.041^{*}$ & 0.321 \\
\hline Cadence (st/min) & $110.67 \pm 5.06$ & $106.19 \pm 6.17$ & $105.05 \pm 5.70$ & $0.011^{*}$ & $0.003^{*}$ & 0.524 \\
\hline Trunk motion (cm) & $3.60 \pm 2.59$ & $3.30 \pm 2.64$ & $2.85 \pm 2.79$ & 0.656 & 0.274 & 0.511 \\
\hline Knee flexion $\left({ }^{\circ}\right)$ & $6.27 \pm 7.55$ & $8.61 \pm 4.84$ & $7.56 \pm 6.66$ & 0.126 & 0.367 & 0.545 \\
\hline Stability & T0 $(n=11)$ & T1 (n=11) & T2 (n=11) & $\begin{array}{c}\text { T0 vs. T1 } \\
\text { p-value }\end{array}$ & $\begin{array}{c}\text { T0 vs. T2 } \\
\text { p-value }\end{array}$ & $\begin{array}{c}\text { T1 vs. T2 } \\
\text { p-value }\end{array}$ \\
\hline $\operatorname{MoS}$ ML (m) & $0.05 \pm 0.01$ & $0.04 \pm 0.01$ & $0.04 \pm 0.01$ & 0.077 & 0.137 & 0.760 \\
\hline $\operatorname{MoS}$ AP (m) & $-0.10 \pm 0.03$ & $-0.09 \pm 0.02$ & $-0.09 \pm 0.03$ & 0.148 & $0.045^{\circ}$ & 0.538 \\
\hline
\end{tabular}

Indicates a statistically significant $(\mathrm{p}<0.05)$. T0 is baseline, T1 is six weeks after baseline, T2 is six months after baseline.

\subsubsection{Radiographic Assessment}

After six weeks of wearing the orthosis patients demonstrated an improvement in static sagittal alignment with respect to baseline as determined by a significant decreased GSA (54.64 \pm 8.49 vs. $48.31 \pm 7.64, \mathrm{p}=0.001$ ) and GAP score (5.64 \pm 2.87 vs. $3.18 \pm 2.86, \mathrm{p}=0.001)$, and decreased SVA ( $50.05 \pm 39.53$ vs. $33.65 \pm 36.37, \mathrm{p}=0.089$ ) (Figure $2 \mathrm{~A}-\mathrm{C}$ ) despite a significantly increased wedge angle $(9.75 \pm 5.03$ vs. $13.69 \pm 4.79, \mathrm{p}<0.001)$ (Figure $2 \mathrm{D})$. However, after six months, when patients stopped wearing the orthosis, a rebound phenomenon was seen

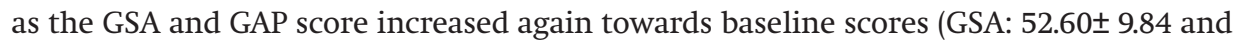
GAP: $5.00 \pm 3.10$ at 6 months) (Figure 2A-C). 
A.

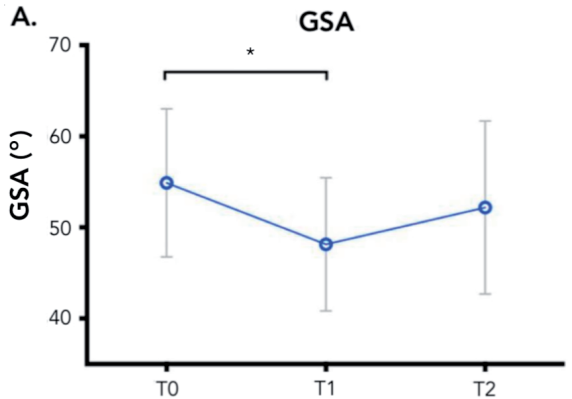

C.

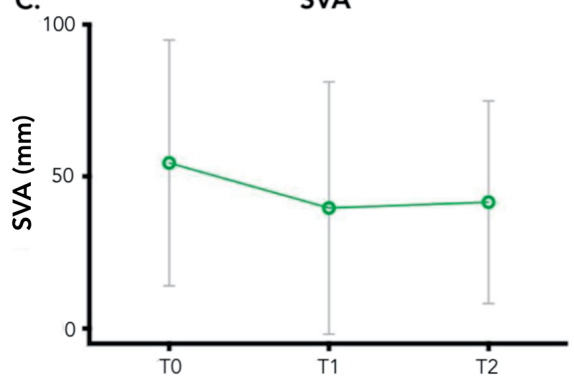

B.

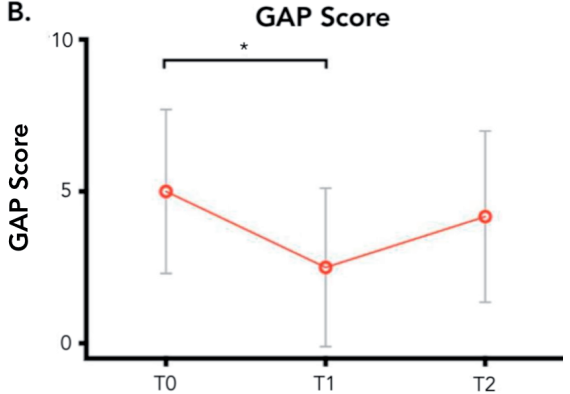

D.

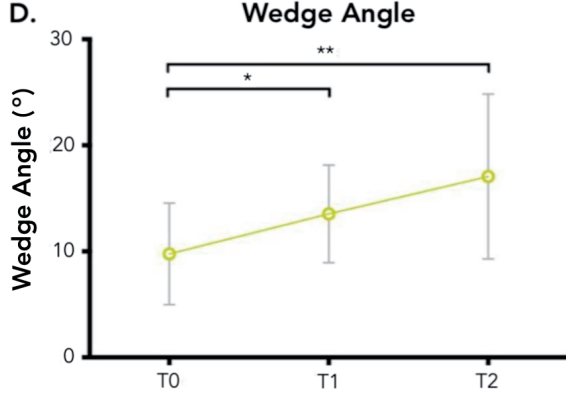

Figure 2. Radiographic sagittal balance.

The graphs represent the mean \pm standard deviation at each time point. A. GSA, significant improvement T0 versus T1 $(\mathrm{p}<0.001)$. Relapse at T2. B. GAP score, significant improvement T0 versus T1 $(\mathrm{p}=0.001)$ ). Relapse at T2. C. SVA, improvement T0 versus T1 ( $\mathrm{p}=0.089)$, Relapse at T2. D. Wedge angle, significant increase at six weeks $(\mathrm{p}<0.001)$ and six months $(\mathrm{p}<0.001)$.

\subsubsection{Quality of Life Assessment}

With regard to baseline, patients reported less pain and improved quality of life six weeks after fracture, showing decreased pain on VAS (68\%, p<0.001), QUALEFFO 41 pain (38\%, $\mathrm{p}<0.001)$, physical function $(42 \%, \mathrm{p}=0.001)$, social function $(21 \%, \mathrm{p}=0.091)$ and general health $(16 \%, \mathrm{p}=0.138)$ scores. These reductions in pain and improvement in quality of life continued after the six weeks visit, showing significantly improved quality of life outcomes at six months compared to baseline. The improvements between six weeks and six months (range 6\%-40\%) were smaller than the improvements observed during the first six weeks (range 16\%-68\%) (Figure 3). 

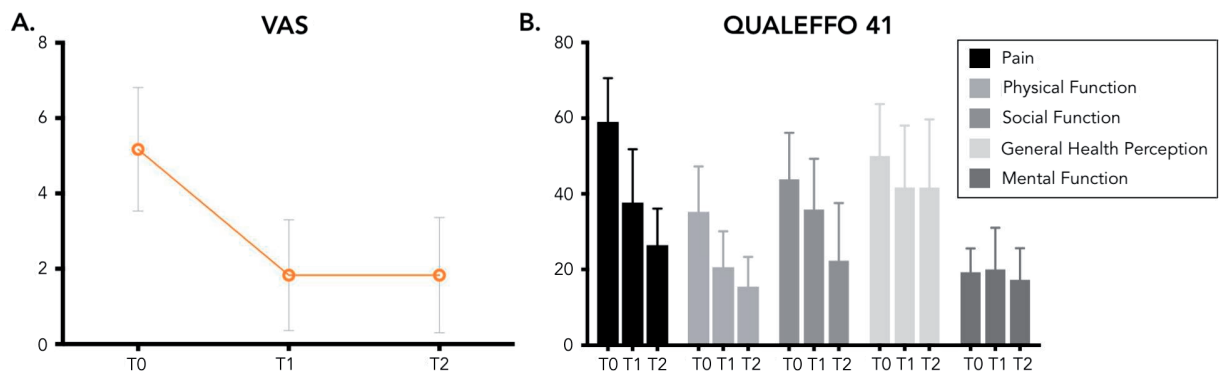

Figure 3. Pain and quality of life.

A. VAS, graphs represent mean \pm standard deviation. Statistically significant improvement in VASscore over time (T0 vs. T1 p<0.001; T0 vs. T2 p<0.001)). B. Qualeffo 41 results, the bars represent the mean \pm standard deviation. Statistically significant improvement over time in Qualeffo domain pain (T0 vs. T1 p<0.001; T0 vs. T2 $\mathrm{p}<0.001$ ), physical function (T0 vs. T1 p=0.001; T0 vs. T2 $\mathrm{p}<0.001$ ), and social function (T0 vs. T1 $\mathrm{p}=0.091 ; \mathrm{T} 0$ vs. T2 $\mathrm{p}<0.001$ ).

\section{DISCUSSION}

In the current study, the use of a semirigid thoracolumbar orthosis had a positive effect on dynamic sagittal balance in patients suffering an OVCF, as was shown at baseline by a significant posterior shift in trunk motion when walking with orthosis compared to walking without orthosis (Table 2). Also, spatiotemporal parameters were positively affected by the orthosis in the majority of patients, however, because of variability these observed changes were not significant at group level (Table 3). After six weeks, static sagittal alignment (radiographs) and trunk motion during walking were improved as compared to baseline. Pain and quality of life improved significantly over the entire study period. Another interesting finding of this study was that it is possible to safely gain detailed information on gait and stability in a frail population with acute pain. A computer-assisted rehabilitation environment allows a safe and standardized condition to accurately analyze gait and stability in frail patients.

The results of this study suggest that six weeks of continuous bracing with a semirigid orthosis results in a more upright posture and adjusted gait pattern, comparable to walking with the orthosis. However, for the improvement in posture and gait pattern, as well as the significant improvement in pain and quality of life the natural healing process of the fractured vertebra might also play an important role. After six months, static and dynamic sagittal alignment deteriorated again. The deterioration in trunk motion may suggest that the improvement in sagittal alignment is slightly reversible. This could be attributed to the fact that patients did not wear the orthosis continuously anymore after six weeks. In contrast, pain and quality of life further improved after six weeks suggesting that the changes in trunk motion are not solely caused by pain. This suggests that the continuous use of a semirigid orthosis might also contribute to the improve- 
ment in sagittal alignment six weeks after baseline [10]. Although the average absolute improvement in sagittal alignment was small, approximately $5^{\circ}$ in radiographic sagittal alignment and 2 centimeters in trunk motion, it is considered clinically relevant. Bruno et al. [24] found that even a small increase in thoracic kyphosis results in a significant increase in vertebral compressive loading. In osteoporosis, the biomechanical strength of the vertebral bodies is reduced, especially in the anterior column [25]. Subsequent compressive overload during loading in flexion causes progressive collapse of the unsupported anterior cortex, which damages the endplates and supporting trabeculae of the vertebrae resulting in novel vertebral fractures [26]. Wei et al. [27], found that vertebral compression fractures are approximately five- to tenfold more prevalent in patients with moderate or severe kyphosis of the thoracolumbar spine in comparison to patients with mild kyphosis. Therefore, in order to minimize increase in spinal loading and prevent new fractures, prevention of an increased thoracolumbar kyphosis is key in patients suffering an OVCF. However, a randomized controlled trial, including a larger patient group is required to further investigate the exact role of dynamic bracing on gait and posture in relation to the natural healing process of the vertebrae.

The improvement in sagittal alignment six weeks after baseline found in the current study might be attributed to an increase in back extensor strength related to the increased antagonistic muscular activity while wearing the orthosis. Pfeifer et al. [12] revealed that wearing an orthosis significantly increases the back extensor strength (72\%) and the abdominal flexor strength (44\%). The increase in muscle strength was correlated with a decreased kyphosis angle and a more upright posture [12]. Valentin et al. [15] demonstrated that dynamic bracing was associated with an increase in back extensor strength of 50\%. This is consistent with the findings of Lantz et al. [28], who showed that wearing an orthosis increases the electrical activity of back muscles. Subsequently, the reversibility of the observed positive effect on sagittal alignment in this study may have been caused by the decline in skeletal muscle strength when patients stopped wearing the orthosis. Without exercise after the age of 30 the muscle mass declines at a rate of three to eight percent each decade due to apoptosis, loss of motor neuron function and a reduction in calcium pumping activity [29]. Therefore, maintaining optimal muscle mass and muscle function by means of active supervised exercise therapy in patients suffering an osteoporotic vertebral fracture may play an important role in the prevention of sagittal malalignment and subsequent fractures. Measuring the actual effect of dynamic bracing on back extensor strength should be considered in future controlled studies.

According to the Global Spine Care Initiative, conservative management of acute pain and recovery of function in adults with OVCFs should include early mobilization, exercise, medication for osteoporosis, spinal orthosis for pain relief only and calcitonin for analgesic-refractory pain [10]. There are no recommendations concerning stability, gait and posture yet. Although preliminary, the results of the current study might indicate that dynamic bracing, using a thoracolumbar spinal orthosis, may be a useful additive in 
the multimodal treatment of OVCFs in order to maintain congruent posture. However, since the effect seemed to be reversible, life-time lumbodorsal muscle exercises should be provided in order to prevent subsequent vertebral fractures [9].

This exploratory study was limited by a relatively small number of patients and did not incorporate a control group. Therefore, the results should be interpreted with caution. However, for the improvement in trunk motion and radiographic sagittal alignment each patient served as their own control. Moreover, a study by Meccariello et al. [13] already revealed a significant reduction in pain and an improvement in quality of life and respiratory function for patients treated with dynamic bracing. For studying the true effectiveness of dynamic bracing in the treatment of OVCFs, a large prospective, randomized controlled trial will be needed. In such a study, a subanalysis should be performed in order to evaluate the effect of fracture site and the degree of compression on the main outcome parameters.

In conclusion, the results of the current study suggest a positive effect of a semirigid thoracolumbar orthosis on gait and stability in patients suffering an OVCF as was shown by a more upright posture, which may result in decreased compressive loading of the vertebrae. In addition, some evidence of a reversible effect on sagittal alignment was provided suggesting the need for prolonged periods of bracing or life-time lumbodorsal muscle exercises. However, the results need to be interpreted with caution as natural recovery following OVCF may play a role. Further research is thus required, preferably by means of a randomized controlled trial including a larger patient group to confirm these results. 


\section{REFERENCES}

1. European Prospective Osteoporosis Study, G, et al. Incidence of vertebral fracture in europe: results from the European Prospective Osteoporosis Study (EPOS). J Bone Miner Res, 2002. 17(4): p. 716-24.

2. Lindsay R, et al. Risk of new vertebral fracture in the year following a fracture. JAMA, 2001. 285(3): p. 320-3.

3. Katzman WB, et al. Age-related hyperkyphosis: its causes, consequences, and management. J Orthop Sports Phys Ther, 2010. 40(6): p. 352-60.

4. Ailon T, et al. Progressive Spinal Kyphosis in the Aging Population. Neurosurgery, 2015. 77 Suppl 4: p. S164-72.

5. Broy SB. The Vertebral Fracture Cascade: Etiology and Clinical Implications. J Clin Densitom, 2016. 19(1): p. 29-34.

6. de Groot $\mathrm{MH}$, et al. A flexed posture in elderly patients is associated with impairments in postural control during walking. Gait Posture, 2014. 39(2): p. 767-72.

7. van der Jagt-Willems HC, et al. Associations between vertebral fractures, increased thoracic kyphosis, a flexed posture and falls in older adults: a prospective cohort study. BMC Geriatr, 2015. 15: p. 34.

8. Sinaki M, et al. Balance disorder and increased risk of falls in osteoporosis and kyphosis: significance of kyphotic posture and muscle strength. Osteoporos Int, 2005. 16(8): p. 1004-10.

9. Sinaki M, et al. Stronger back muscles reduce the incidence of vertebral fractures: a prospective 10 year follow-up of postmenopausal women. Bone, 2002. 30(6): p. 836-41.

10. Ameis A, et al. The Global Spine Care Initiative: a review of reviews and recommendations for the non-invasive management of acute osteoporotic vertebral compression fracture pain in low- and middle-income communities. Eur Spine J, 2017.

11. Rzewuska M, et al. The efficacy of conservative treatment of osteoporotic compression fractures on acute pain relief: a systematic review with meta-analysis. Eur Spine J, 2015. 24(4): p. 702-14.

12. Pfeifer M, et al. Effects of two newly developed spinal orthoses on trunk muscle strength, posture, and quality-of-life in women with postmenopausal osteoporosis: a randomized trial. Am J Phys Med Rehabil, 2011. 90(10): p. 805-15.

13. Meccariello L, et al. Dynamic corset versus three-point brace in the treatment of osteoporotic compression fractures of the thoracic and lumbar spine: a prospective, comparative study. Aging Clin Exp Res, 2017. 29(3): p. 443-449.

14. Azadinia F, et al. The effects of two spinal orthoses on balance in elderly people with thoracic kyphosis. Prosthet Orthot Int, 2013. 37(5): p. 404-10.

15. Valentin GH, L.N. Pedersen, and T. Maribo. Wearing an active spinal orthosis improves back extensor strength in women with osteoporotic vertebral fractures. Prosthet Orthot Int, 2014. 38(3): p. 232-8.

16. Kim HJ, et al. Comparative study of the treatment outcomes of osteoporotic compression fractures without neurologic injury using a rigid brace, a soft brace, and no brace: a prospective randomized controlled non-inferiority trial. J Bone Joint Surg Am, 2014. 96(23): p. 1959-66.

17. McCrum C, et al. Associations Between Bipedal Stance Stability and Locomotor Stability Following a Trip in Unilateral Vestibulopathy. J Appl Biomech, 2017. 33(2): p. 112-117. 
18. Hak L, et al. Speeding up or slowing down?: Gait adaptations to preserve gait stability in response to balance perturbations. Gait Posture, 2012. 36(2): p. 260-4.

19. Hak L, et al. Stepping strategies for regulating gait adaptability and stability. J Biomech, 2013. 46(5): p. 905-11.

20. Zeni JA, Jr., J.G. Richards, and J.S. Higginson. Two simple methods for determining gait events during treadmill and overground walking using kinematic data. Gait Posture, 2008. 27(4): p. 710-4.

21. Hof AL, M.G. Gazendam, and W.E. Sinke. The condition for dynamic stability. J Biomech, 2005. 38(1): p. 1-8.

22. Smith MW, et al. Acute proximal junctional failure in patients with preoperative sagittal imbalance. Spine J, 2015. 15(10): p. 2142-8.

23. Yilgor C, et al. Global Alignment and Proportion (GAP) Score: Development and Validation of a New Method of Analyzing Spinopelvic Alignment to Predict Mechanical Complications After Adult Spinal Deformity Surgery. J Bone Joint Surg Am, 2017. 99(19): p. 1661-1672.

24. Bruno AG, et al. The effect of thoracic kyphosis and sagittal plane alignment on vertebral compressive loading. J Bone Miner Res, 2012. 27(10): p. 2144-51.

25. Hou Y and Z Luo, A study on the structural properties of the lumbar endplate: histological structure, the effect of bone density, and spinal level. Spine (Phila Pa 1976), 2009. 34(12): p. E427-33.

26. Landham PR, et al. Pathogenesis of Vertebral Anterior Wedge Deformity: A 2-Stage Process? Spine (Phila Pa 1976), 2015. 40(12): p. 902-8.

27. Wei Y, et al. Thoracolumbar kyphosis is associated with compressive vertebral fracture in postmenopausal women. Osteoporos Int, 2017.

28. Lantz SA and AB Schultz. Lumbar spine orthosis wearing. II. Effect on trunk muscle myoelectric activity. Spine (Phila Pa 1976), 1986. 11(8): p. 838-42.

29. Flack KD, et al. Aging, resistance training, and diabetes prevention. J Aging Res, 2010. 2011: p. 127315. 




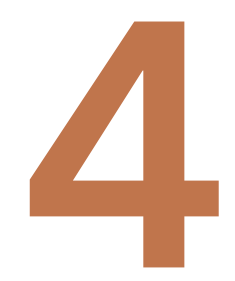

Synthesis and

characterization of a new vertebroplasty cement based on gold-containing PMMA microspheres

Jacobs E, Saralidze K, Roth AK, Jong JJA, van den Bergh JPW, Knetsch MLW, Brans B, Lataster A, Dkordevic I, Willems PCPH, Koole LH Biomaterials. 2016; 82: 60-70 


\section{ABSTRACT}

There are a number of drawbacks to incorporating large concentrations of barium sulphate $\left(\mathrm{BaSO}_{4}\right)$ as the radiopacifier in PMMA-based bone cements for percutaneous vertebroplasty. These include adverse effects on injectability, viscosity profile, setting time, mechanical properties of the cement and bone resorption. We have synthesized a novel cement that is designed to address some of these drawbacks. Its powder includes PMMA microspheres in which gold particles are embedded and its monomer is the same as that used in commercial cements for vertebroplasty. In comparison to one such commercial cement brand, VertaPlex ${ }^{\mathrm{TM}}$, the new cement has longer doughing time, longer injection time, higher compressive strength, higher compressive modulus, and is superior in terms of cytotoxicity. For augmentation of fractured fresh-frozen cadaveric vertebral bodies (T6-L5) using simulated vertebroplasty, results for compressive strength and compressive stiffness of the construct and the percentage of the volume of the vertebral body filled by the cement were comparable for the two cements although the radiopacity of the new cement was significantly lower than that for VertaPlex ${ }^{\mathrm{TM}}$. The present results indicate that the new cement warrants further study. 


\section{INTRODUCTION}

Osteoporotic vertebral compression fractures are the most common fractures among the elderly, with an estimated number of 1.4 million new fractures occurring every year in patients over 50 years of age worldwide [1-3]. Although some vertebral compression fractures can be asymptomatic, most patients experience substantial, incapacitating pain, which can become chronic [3,4]. Percutaneous vertebroplasty is a minimally invasive procedure in which bone cement is carefully injected into a painful osteoporotic fractured vertebra under continuous fluoroscopic guidance. The bone cement hardens in situ, providing increased strength and stabilization. Most importantly, pain is substantially relieved which, in turn, results in a significant improvement of quality of life in the elderly population $[3,4]$.

The bone cement used for vertebroplasty are derived from conventional polymethylmethacrylate (PMMA) bone cements used for total hip and knee arthroplasty. The main modification is that cements used in vertebroplasty contain a much higher proportion of radiopacifier (up to $30 \%$ on weight basis), most commonly barium sulphate $\left(\mathrm{BaSO}_{4}\right)$ [5-7]. Large amounts of radiopacifier particles negatively influence injectability of the cement as the particles have a tendency to form agglomerates due to a higher molecular weight and polarity in comparison to PMMA [8]. Kurtz et al. [8] revealed that these large agglomerations of $\mathrm{BaSO}_{4}$ also act as fatigue initiation sites, and adversely affect the fatigue life of the cement. Moreover, the mechanical properties of the cement relevant to its use in vertebroplasty (that is, compressive strength and fracture toughness) are reduced due to increased concentrations of radiopacifier [9]. Furthermore, in vitro and in vivo histological studies have shown that the conventional radiopacifying agents evoke a pathological response. $\mathrm{BaSO}_{4}$ is known to cause a foreign body inflammatory reaction, activating macrophages and pro-inflammation cytokines which, in turn, leads to the onset of osteolysis [8-11].

Commercial PMMA bone cements start to polymerize at a rapid rate immediately after the powder and liquid fractions are mixed. This exothermic reaction leads to a large increase in cement viscosity over a few minutes with a short handling time as a consequence. In a bid to improve handling properties of a cement (increase its injection and setting times), it is common for surgeons to alter the powder-to-liquid ratio that is recommended by the cement manufacturer. By doing so, a lower viscosity can be obtained, which may offer more time and more control during injection because less force is needed [12]. On the other hand, a major concern is the risk of cement leakage during injection, which increases with lower cement viscosity [13]. Complications associated with cement extravasation, such as pulmonary cement embolism and nerve root compression have been reported $[3,4,13]$.

One way to control and improve injectability and setting times is to incorporate crosslinked PMMA microspheres in the polymer phase. Rodrigues et al. [12] showed that by 
using these microspheres, handling behaviour can be substantially improved: viscosity was decreased during a prolonged injection window, whereas the maximum exotherm temperature was reduced significantly to provide protection from heat damage. By incorporating the radiopacifier within these cross-linked microspheres, negative effects of the radiopacifier will also be diminished. Since agglomerates of the radiopacifier that impede cement injection through a needle can no longer be formed, injectability may be improved further. Since both the microspheres and the surrounding matrix are both comprised of PMMA, the cement should have similar mechanical properties to the currently used commercial cements. Additionally, as radiopacifier particle leach-out is highly improbable, tissue and inflammatory responses induced by the contrast agent will be limited [8-11].

There is an ongoing debate regarding the suitability of $\mathrm{BaSO}_{4}$ as radiopacifier in bone cements [8-13]. The incorporation of several alternative radiopacifier agents (for example, iodine, bismuth salicylate, and zinc) has been investigated extensively [14-20]. Ideally, a radiopacifier for percutaneous vertebroplasty should be inert, free from any contaminants, and non-toxic. Gold particles as radiopacifying agent have been used for dental root canal sealants and in-cast restorations [21,22]. As shown by Camilleri et al. [21], gold exhibits high radiopacity values due to its high relative atomic mass. Furthermore, gold particles have excellent biocompatibility and facile synthesis [20,22]. All of these properties make gold particles an excellent candidate as a radiopacifier specifically for incorporation within microspheres.

In the present work, we studied a conceptually new type of cement, comprising gold particle-containing PMMA microspheres, which may be suitable for vertebroplasty. Although the formulation of the new cement is comparable to that of commercial cements for vertebroplasty, there is one major difference: the gold particles were not just added to the solid portion of the cement, prior to cement mixing. Rather, the new cement was based on synthesis of cross-linked PMMA microspheres in which gold particles were embedded homogeneously. In a commercial cement for vertebroplasty, the dough consists of a continuous phase (largely, MMA monomer) and two dispersed phases (PMMA microspheres and irregular $\mathrm{BaSO}_{4}$ particles). However, in the new cement, the dough consists of the same continuous phase (largely, MMA monomer) and one dispersed phase: the crosslinked PMMA microspheres with gold particles incorporated. The study had two purposes. The first was to compare the novel cement and a counterpart commercial cement brand used for vertebroplasty on the basis of doughing time, injection time, cytocompatibility, compressive strength and compressive modulus. The second was to compare these two cements when used to augment fractured fresh-frozen cadaveric vertebral bodies (T6-L5) using simulated vertebroplasty on the basis of compressive strength and stiffness of the construct, the percentage of the volume of the vertebral body filled by the cement, and radiopacity of the cement. 


\section{MATERIALS AND METHODS}

\subsection{Microsphere Synthesis and Cement Preparation}

\subsubsection{Materials}

Chemicals were purchased from Sigma/Aldrich/Fluka (Zwijndrecht, The Netherlands) and Acros (Landsmeer, the Netherlands). Benzoyl peroxide (BPO), tetraethylene glycol dimethacrylate (TEGDMA), sodium chloride ( $\mathrm{NaCl})$, glutaraldehyde (25\% solution), poly(methyl-methacrylate) (PMMA; M.W. 120,000), poly(vinyl alcohol) (PVA; M.W. 86,000; 99-100\% hydrolyzed), poly(ethyleneglycol) (PEG; M.W. 1,000), poly(vinyl pyrrolidone) (PVP; K-23-32; M.W. 58,000), MTT, and hexamethyl-disilazane were used as purchased. Methylmethacrylate (MMA) was distilled at atmospheric pressure and stored at $-20^{\circ} \mathrm{C}$. Gold powder was purchased from Metalor ${ }^{\circledR}$ (PUAU3335; Neuchâtel, Switzerland); particle size distribution: $90 \%<2.7 \mu \mathrm{m}, 50 \%<1.5 \mu \mathrm{m}, 10 \%<0.8 \mu \mathrm{m}$. Cell culture medium (DMEM-F12), fetal bovine serum (FBS), antibiotics, and Cyquant reagent were purchased from Life Technologies (Bleiswijk, The Netherlands). For comparison, a commercial vertebroplasty cement brand, VertaPlex ${ }^{\text {TM }}$ (Stryker, Kalamazoo, MI, USA) was used consisting of $9.5 \mathrm{~mL}$ of a liquid monomer (9.4 mL MMA, $0.10 \mathrm{~mL} \mathrm{~N}$,N-dimethyl-para-toluidine and $0.75 \mathrm{mg}$ hydroquinone) and $20 \mathrm{~g}$ of a finely divided powder (6.0 $\mathrm{g} \mathrm{BaSO}_{4}$ (30 wt./wt\%), $14.0 \mathrm{~g}$ PMMA containing benzoylperoxide (2.6\%)). The Viscosafe Injection Kit (syringes and accessories for vertebroplasty), manufactured by Synthes (Oberndorf, Switzerland), was used in all the experiments.

\subsubsection{Synthesis of Gold-containing_PMMA Microspheres}

An aqueous solution $(150 \mathrm{~mL})$ of poly(vinyl alcohol) (3.5 g), poly(ethylene glycol) (4.0 g), and poly(N-vinyl pyrrolidinone) (2.0 g) was prepared in a $250-\mathrm{mL}$ round bottom flask. The solution was magnetically stirred $(1100 \mathrm{rpm})$ and heated to $86-87^{\circ} \mathrm{C}$. A suspension of methyl methacrylate (11.55 g, $115.36 \mathrm{mmol})$, gold particles $(2.00 \mathrm{~g})$, benzoyl peroxide (500 $\mathrm{mg}, 2.06 \mathrm{mmol}$ ), tetraethyleneglycoldimethacrylate (404 mg, $1.22 \mathrm{mmol})$, N,N-dimethylp- toluidine (94 mg, $0.69 \mathrm{mmol}$ ), sodium chloride ( $2.5 \mathrm{~g}, 42.78 \mathrm{mmol}$ ), and PMMA (3.24 g; M.W. 120,000) was prepared and thoroughly mixed in a 100-mL round bottom flask. The viscous slurry was poured into the hot and stirred aqueous solution. Heating and stirring were continued for 4 hours. Formation of microspheres was confirmed by taking an aliquot, and examining this by light microscopy. Then, the heat source was turned off, and the flask was allowed to cool to room temperature. After several hours, stirring was stopped and the microspheres were allowed to settle on the bottom of the flask. The microspheres were isolated by centrifugation (200 g), washed with water (five times), and lyophilized by freeze-drying. The microspheres were size-sorted by sieving in two classes, 300 - $600 \mu \mathrm{m}$ diameter, and 0 - $300 \mu \mathrm{m}$ diameter, using calibrated circular sieves with a diameter of $20 \mathrm{~cm}$ (Retsch, Germany). Microspheres without gold particles were 
also synthesized, using the identical protocol for radiolucent cement. The purpose of this radiolucent PMMA microsphere control cement was to be able to assess the effect of contrast agents on the cement performance in the different in vitro experiments. For the ex vivo experiments this control cement was not used because of costs and limited availability of human donor spines.

\subsubsection{Cement Preparation}

Gold-containing microspheres with a diameter in the range $0-300 \mu \mathrm{m}(4.00 \mathrm{~g})$ were transferred into a bowl of aluminum foil, and $110 \mathrm{mg}$ of benzoyl peroxide was added. A stock solution of methyl methacrylate and N,N-dimethyl-p-toluidine (40.0:0.50; g:g) was made in a closed 100-mL Erlenmeyer flask. $4.00 \mathrm{~g}$ of this mixture was transferred into the aluminum bowl. The powder and liquid fractions were mixed with a spatula for several minutes, until formation of a dough. At this time, the microspheres were suspended in the dough and no longer sank to the bottom. The VertaPlex ${ }^{\mathrm{TM}}$ control cement was mixed according to the manufacturer's instructions. For the radiolucent cement, the protocol for preparing the gold cement was used. The time until a usable dough was obtained was recorded. When reaching dough consistency, the cements were carefully inserted into 5-mL polypropylene syringes (4 g per syringe) from which the plunger was removed. The plunger was re-inserted and enclosed air bubbles were removed. The time at which the syringes became $>70^{\circ} \mathrm{C}$, indicating that the polymerization reaction took place and injection was no longer possible, was recorded. The filled syringes were left untouched for 24 hours. Note that curing of the cement samples occurred below the glass transition temperature of PMMA (which is $105^{\circ} \mathrm{C}$ approximately). This implies that the polymerization is diffusion-controlled, and that conversion will not reach $100 \%$. These conditions resemble those in vivo; it is well known that elevated temperatures due to the exothermic nature of the polymerization also occur in vivo. The cement rods were machined into test samples, either cement discs of $1.0 \mathrm{~mm}$ height and $6.0 \mathrm{~mm}$ diameter (for in vitro cytocompatibility), or cylinders of $6.0 \mathrm{~mm}$ height and $6.0 \mathrm{~mm}$ diameter (for uniaxial compression).

Scanning electron microscopy (SEM) was performed with a Philips XL-30 instrument. Fundamental insight into the morphology of the cement was attained using backscatter mode at an accelerating voltage of $30 \mathrm{kV}$. Cements discs were cut and polished, and surfaces were sputter-coated with carbon prior to examination.

\subsection{In Vitro Cytocompatibility Analysis}

\subsubsection{Cell Culture}

Mouse fibroblasts (L929) were used for evaluating cytotoxicity of cement extracts. For the evaluation of the direct contact between cells and cements, both L929 and SAOS-2 cells (an osteosarcoma cell line) were used [23]. DMEM/F12, containing Glutamax, supple- 
mented with $10 \%(\mathrm{v} / \mathrm{v})$ fetal bovine serum (FBS) and antibiotics $(10 \mathrm{U} / \mathrm{mL}$ penicillin, 10 $\mu \mathrm{g} / \mathrm{mL}$ streptomycin, and $0.25 \mu \mathrm{g} / \mathrm{mL}$ amphotericin B) was used as culture medium for L929 mouse fibroblasts. MEM with Glutamax containing the identical supplements, as described for the DMEM/F12 medium, was used for the SAOS-2 cells. Cells were grown at $37^{\circ} \mathrm{C} / 5 \% \mathrm{CO}_{2}$ and harvested with Trypsin/EDTA $(0.05 \% / 0.4 \mathrm{mM})$. Cells were counted using a haemocytometer and inverted light microscope (Leica DM-IL) and diluted to the desired cell density.

\subsubsection{Cytotoxicity of Cement Extracts}

Cement discs were sterilized by incubation with ethanol and subsequent evaporation in a sterile environment. Samples were then incubated in medium $(0.2 \mathrm{~g} / \mathrm{mL})$ at $37^{\circ} \mathrm{C}$ for 48 hours (first extract) and subsequently in fresh medium for 72 hours (second extract). 10,000 cells were added to the wells of a 96-well plate and incubated for 24 hours at $37^{\circ} \mathrm{C} / 5 \% \mathrm{CO}_{2}$. The medium was removed and replaced with $100 \mu \mathrm{L}$ of the first or second extract (vide supra). Medium without cement sample and medium with $5 \%$ dimethylsulfoxide (DMSO) were used as controls. The cells were incubated for another 48 hours with the extracts. $25 \mu \mathrm{L}$ of medium containing MTT $(2 \mathrm{mg} / \mathrm{mL})$ was added, and the plates were further incubated at $37^{\circ} \mathrm{C}$ for 90 minutes. The medium was aspirated, the formed purple formazan was dissolved in isopropanol, and absorbance at $570 \mathrm{~nm}$ was measured. Cell viability was expressed as percentage of the negative control (medium without cement sample).

\subsubsection{Direct Method}

The discs, which showed to be free of toxic leachables, were seeded with two different cell types. The L929 was used as a bio-sensing cell line, relatively sensitive to toxic compounds and toxic surfaces. The SAOS-2 cells, related to osteoblasts, represent the type of cells that most likely will interact with these cements in situ.

Thoroughly washed cement discs were sterilized with alcohol and put in the wells of a 24-well plate. Approximately $80 \mu \mathrm{L}$ of cell suspension $\left(1.10^{6}\right.$ cells $\left.\mathrm{mL}^{-1}\right)$ was carefully put on top of the cement discs. The cells were allowed to adhere to the surface for one hour at $37^{\circ} \mathrm{C}$, after which enough medium was added to the wells to cover the surface $(1.0 \mathrm{~mL})$. The plate was left for 72 hours at $37^{\circ} \mathrm{C} / 5 \% \mathrm{CO}_{2}$. Subsequently, the cells were washed once in warm PBS and stained for 20 minutes at room temperature using the Live/Dead assay from Life technologies (L-3224). The final concentrations used were 2 $\mu \mathrm{M}$ Calcein-AM and $4 \mu \mathrm{M}$ Ethidium homodimer I. The samples were observed using a Nikon Eclipse E-800 fluorescence microscope equipped with an Evolution-VF cooled CCD camera (Media Cybernetics, Rockville, MD, USA). Living cells appeared green while dead cells turned fluorescent red.

Alternatively, samples were placed in fixative (2.5\% glutaraldehyde in PBS), after washing in PBS, overnight at $4^{\circ} \mathrm{C}$. Next the samples were washed with PBS (three times) and 
dehydrated with an ethanol series (50, 70, 80, 90, 96, 100\%). The samples were then incubated for three minutes in hexamethyl-disilazane and subsequently air-dried [24]. These samples were then prepared for SEM analysis by gluing cement discs with attached cells to sample holders using silver glue, and sputter-coating with gold. Cell morphology was studied by imaging in the standard mode at an accelerating voltage of $10 \mathrm{kV}$. Glass plates were used as a control for easy identification of cell morphology because some cement discs may have debris on them and/or were not perfectly flat, which may make identification of cells difficult.

\subsection{Biomechanical Testing}

\subsubsection{Uniaxial Compression}

Uniaxial compression tests were carried out using an Instron Automated Materials Testing System (LX Model) equipped with a $10 \mathrm{kN}$ loading cell. The tests were driven at ambient temperature. Cylindrical cement samples with height of $6 \mathrm{~mm}$ and diameter of $6 \mathrm{~mm}$ were used. No blocking of transversal strains could occur since the samples were placed freely between the support surfaces of the testing machine. Samples were tested at a compression rate of $1 \mathrm{~mm} / \mathrm{min}(\mathrm{n}=8-10)$. Compression was stopped at $50 \%$ compression (height $=3 \mathrm{~mm}$ ).

The modulus and strength ( $0.2 \%$ offset) were calculated from the slope of the linear part of the stress/strain diagram i.e. at displacement $<5 \%$ (Figure 1). Experiments were performed in eight-fold (for radiolucent control cement) or performed ten-fold for the gold-containing cement and for VertaPlex ${ }^{\mathrm{TM}}$.

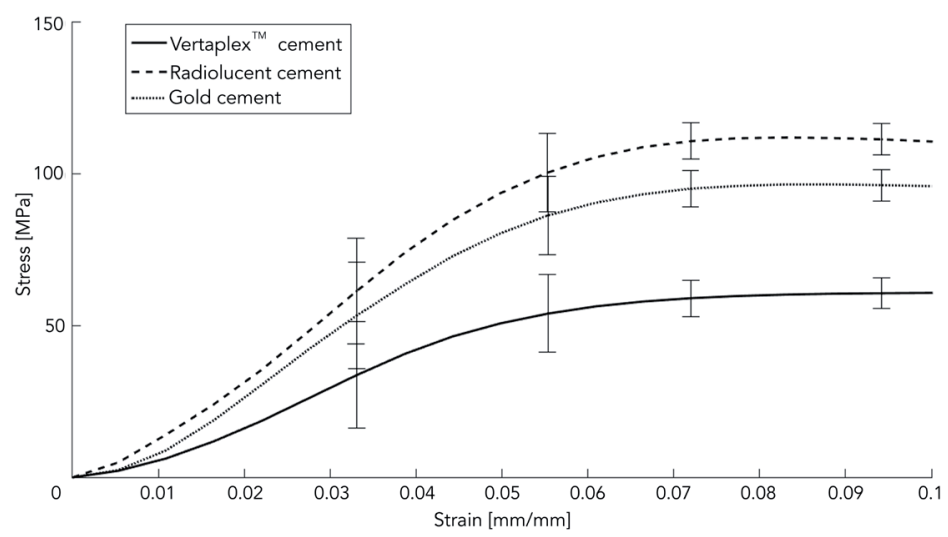

Figure 1. Set of representative experimental stress-strain curves (mean \pm standard deviation represented as error bars) after uniaxial compression. 


\subsubsection{Cement Injection into Human Cadaveric Vertebrae with Simulated}

\section{Compression Fractures}

The mechanical response of vertebrae with simulated osteoporotic vertebral compression fractures (OVCF's), before and after injection with two cements (VertaPlex ${ }^{\mathrm{TM}}$ versus gold-containing microsphere cement), were compared.

\subsubsection{Vertebral Anatomy}

Twenty-two intact human vertebrae (T6-L5) were obtained and isolated from two fresh frozen female cadavers (86 and 89 years of age) which had been donated for medical research (Department of Anatomy and Embryology, Maastricht University). Spines were kept frozen $\left(-20^{\circ} \mathrm{C}\right)$ in sealed bags. The areal bone mineral density (aBMD) was determined for each spine by dual energy X-ray absorptiometry. Computed tomography evaluation was performed to rule out vertebral fractures. After thawing at room temperature $\left(20^{\circ} \mathrm{C}\right)$ for at least 24 hours, individual vertebrae were separated and excised of soft tissue. The posterior elements (spinous process and vertebral arch) were removed to facilitate mechanical testing, leaving the pedicles intact. Each vertebra was floated in a sealed plastic bag in a water bath maintained at $37^{\circ} \mathrm{C}$ for at least one hour prior to testing to ensure that the vertebrae were at body temperature.

Within each of the spines, consecutive vertebrae were considered paired specimens; that is, T6 and T7 constituted one pair and L4 and L5 constituted another pair. One of each paired members was assigned into the experimental group (gold-containing microsphere cement), while the other paired member was assigned to the (VertaPlex ${ }^{\mathrm{TM}}$ cement) control group. Assignment into either group was alternated for paired vertebrae from the second donor in order to avoid potential bias that is associated with assigning the superior vertebra of each pair to the same experimental group [25].

\subsubsection{Simulated Osteoporotic Vertebral Compression Fracture Model}

Impressions of the inferior and superior endplates of each vertebra were made by pouring two blocks of polymethylmethacrylate (PMMA) microtome mounting medium (Technovit 3040, Heraeus Kulzer, Haarlem, The Netherlands) on both sides of the vertebral body to provide homogenous loading and standardized positioning of the vertebrae. The vertebral bodies, seated between these two blocks, were then placed in between two platens on a materials testing machine (MTS actuator, Zwick/Roell, ZMART PRO) for determination of initial strength and stiffness. Initial stiffness was defined as the average slope on the load-displacement curve. Strength was defined as the inflection point on the load-displacement curve (Figure 2) [25]. Pure axial compression was ensured by means of a ball-and-socket system in the top platen to induce a compression fracture. Each vertebra was preloaded in axial compression to $80 \mathrm{~N}$ for 60 seconds after which compression was applied to the superior plate at a rate of $5 \mathrm{~mm} / \mathrm{min}$, until the anterior 
height of the vertebrae was decreased by $25 \%[25,26]$. Because of the adjustable load application via the ball-and-socket system a wedge-shaped compression fracture was provoked [25,26].

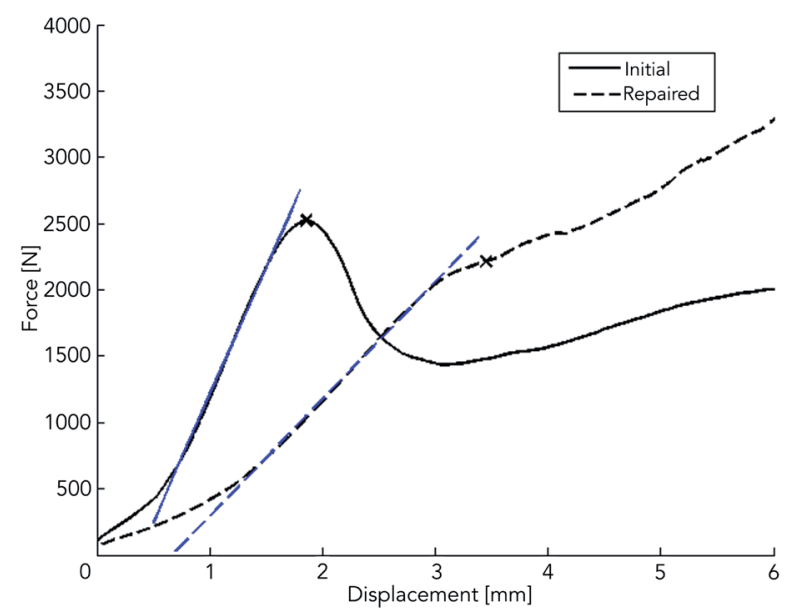

Figure 2. Typical initial and post-cement augmentation mechanical response of a vertebral body [25].

Stiffness was defined as the average slope and strength as the inflection point on the load-displacement curve.

\subsubsection{Cement Injection}

After the simulated (wedge-shaped) compression fracture was created, a 12-gauge vertebroplasty injection needle was inserted under biplanar fluoroscopic control through each pedicle base into the fractured vertebral body (Figure 3). The assigned cement was prepared as described in paragraph 2.1.3. Cements were hand-mixed using regular cement mixing equipment for one or five minutes (VertaPlex ${ }^{\mathrm{TM}}$ versus gold-containing microsphere cement, respectively), and then transferred into the syringes.

Each of the cements was injected bipedicularly under lateral C-arm fluoroscopic control by an experienced senior spine surgeon (PW). Injection was stopped if (pending) posterior extravasation was observed on fluoroscopy. At thoracic levels, a maximum volume of 6 $\mathrm{mL}$ of the appropriate cement was injected into each vertebra and a maximum volume of $8 \mathrm{~mL}$ was injected into the lumbar vertebrae [27]. In order to mimic body temperature, the vertebrae were sealed in plastic bags and floated in a bath maintained at $37^{\circ} \mathrm{C}$ for 24 hours after cement augmentation. The vertebral specimens were then compressed again according to the initial fracture protocol until failure, and post-augmentation strength and stiffness were determined as before. 

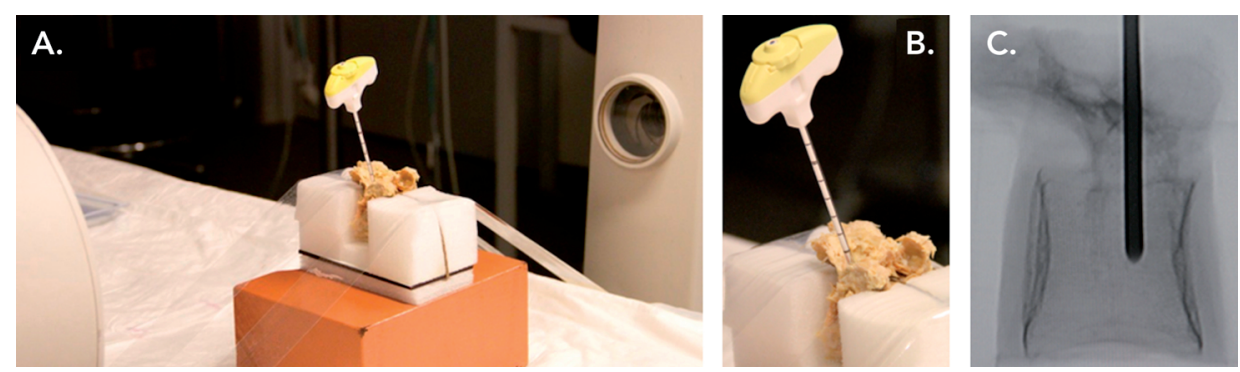

Figure 3. Test set-up.

A. Placement of a vertebra in the bundle of the fluoroscopic guidance system (C-arm). One trocar has been inserted (left pedicle); B. Detailed image of the trocar; C. Example of a real-time fluoroscopy image, showing correct placement of the trocar's tip at the center of the vertebra.

\subsubsection{Cement Distribution and Radiopacity}

High resolution peripheral quantitative computed tomography (HR-pQCT, Xtreme CT, Scanco Medical AG, Switzerland) imaging of the vertebrae was performed before the initial compression, after the initial compression and after cement augmentation in order to assess radiopacity and filling percentage and distribution of the cement in the vertebral bodies. Standard scan settings were used (peak voltage of $60 \mathrm{kVp}$, tube current of $900 \mu \mathrm{A}$ and $100 \mathrm{~ms}$ integration time) and images were reconstructed using an isotropic voxel size of $82 \mu \mathrm{m}$. The apparent volumetric bone mineral density $\left[\mathrm{mgHA} / \mathrm{cm}^{3}\right.$ ] was evaluated for the cortical and trabecular compartments of the vertebrae. Apparent density was also used as a measure of radiopacity of the cement.

Fill percentage was determined using a custom written script (MATLAB; MathWorks, Natick, MA, USA). After applying a Gaussian filter, thresholding was applied to distinguish between cement and bone. Total bone volume was acquired by filling in the trabecular bone structure using repeated dilation and erosion steps [27].

\subsubsection{Statistics}

All of the quantitative results were analyzed using SPSS version 19 for Windows (SPSS, Inc., Chicago, IL). The cytotoxicity data were checked for normality using the Shapiro/ Wilk test $(\mathrm{p}>0.05)$. All the data passed this test and were further analyzed using a paired T-test. Differences in measured parameters between the VertaPlex ${ }^{\mathrm{TM}}$ cement group and the gold-containing microsphere cement group were analyzed with a Mann-Whitney $\mathrm{U}$ test (significance level at $\mathrm{p}<0.05$ ). In order to investigate the differences in strength and stiffness within each experimental group, a Wilcoxon signed-ranks test was used (significance level at $\mathrm{p}<0.05$ ). 


\section{RESULTS}

\subsection{Microsphere Synthesis and Cement Preparation}

\subsubsection{Characteristics of Microspheres}

The yield of the gold-containing PMMA microspheres, from the suspension, was $>90 \%$. The inclusion of PMMA (M.W. 120,000) in the mix of monomers and gold particles was required to obtain efficient synthesis of microspheres containing gold particles. The added PMMA increased the viscosity of the monomer/gold mix and, thus, prevented the loss of gold particles because of the centrifugal force from the non-polymerized monomer droplets in the surfactant solution. The gold-containing microspheres were sieved and those within the diameter range of $0-300 \mu \mathrm{m}$ were collected for use in the experimental cement. The yield of gold-containing microspheres in the range of $0-300$ $\mu \mathrm{m}$ was approximately $50 \mathrm{wt} \%$, with the other $50 \mathrm{wt} \%$ being microspheres with a large diameter.

\subsubsection{Cement Properties}

For VertaPlex ${ }^{\mathrm{TM}}$, the doughing time (median: $3.0 \mathrm{~min}$; range: $2.0-4.5 \mathrm{~min}$ ) was significantly shorter than for the gold-containing cement (median: $7.0 \mathrm{~min}$; range: 6.0-9.0 min), with the trend in injection time being the same (6-8 $\mathrm{min}$ and 14-16 min) for VertaPlex ${ }^{\mathrm{TM}}$ and the gold-containing cement, respectively.

The fundamental structural difference between the VertaPlex ${ }^{\mathrm{TM}}$ cement and the goldcontaining microsphere cement can easily be observed using backscatter SEM of cement samples (Figure 4). In the commercial VertaPlex ${ }^{\mathrm{TM}}$ cement, the most electron dense material $\left(\mathrm{BaSO}_{4}\right)$ is found in the matrix surrounding the dark PMMA spheres (Figure 4B). The $\mathrm{BaSO}_{4}$ particles become dispersed in the liquid MMA upon mixing and trapped in the PMMA matrix after polymerization. In the gold-containing microsphere cement,
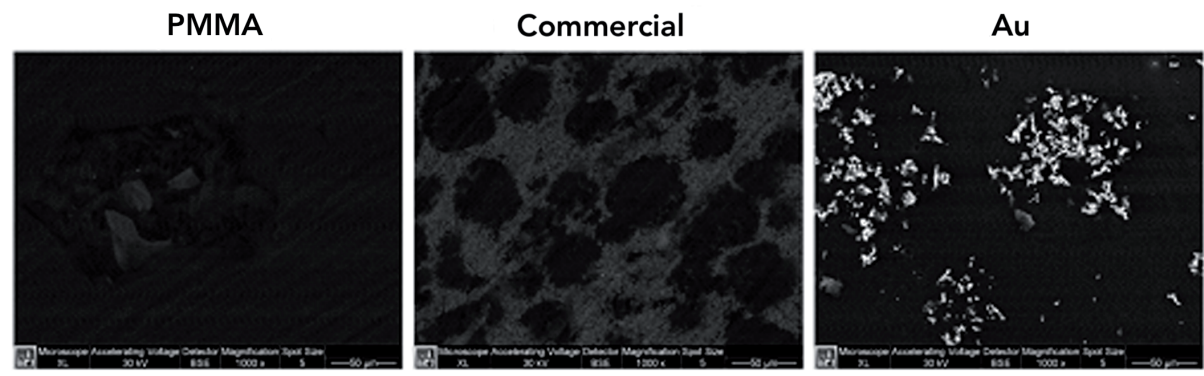

Figure 4. Backscatter SEM images of cement surfaces.

The radiopacifier $\mathrm{BaSO}_{4}$ is observed between the microspheres of the VertaPlex ${ }^{\mathrm{TM}}$ cement. In the gold-containing microsphere cement the gold particles can be easily distinguished, also showing the outline of the microspheres. 
the gold particles are incorporated in the microspheres, leading to localized patches of dense material (Figure $4 \mathrm{C}$ ). The borders between the microspheres and the PMMA matrix cannot be clearly observed since both have identical electron density and, thus, identical color in the backscatter images. Still, it is possible to see the contours of the gold-containing microspheres in the cement by the localization of the electron dense gold particles.

\subsection{Cytocompatibility}

\subsubsection{Cytocompatibility of Extracts from Cements}

In Figure 5 is shown the level of cytotoxicity of the leachables from both cements. It is clear that only the first extract from the VertaPlex ${ }^{\mathrm{TM}}$ cement shows some moderate cytotoxicity (50\% cell viability). The second extract from this cement shows a cell viability of just over 75\%, with the cells in the wells having normal morphology. The newly synthesized gold-containing microsphere cement shows no cytotoxicity, as neither does the radiolucent control cement.

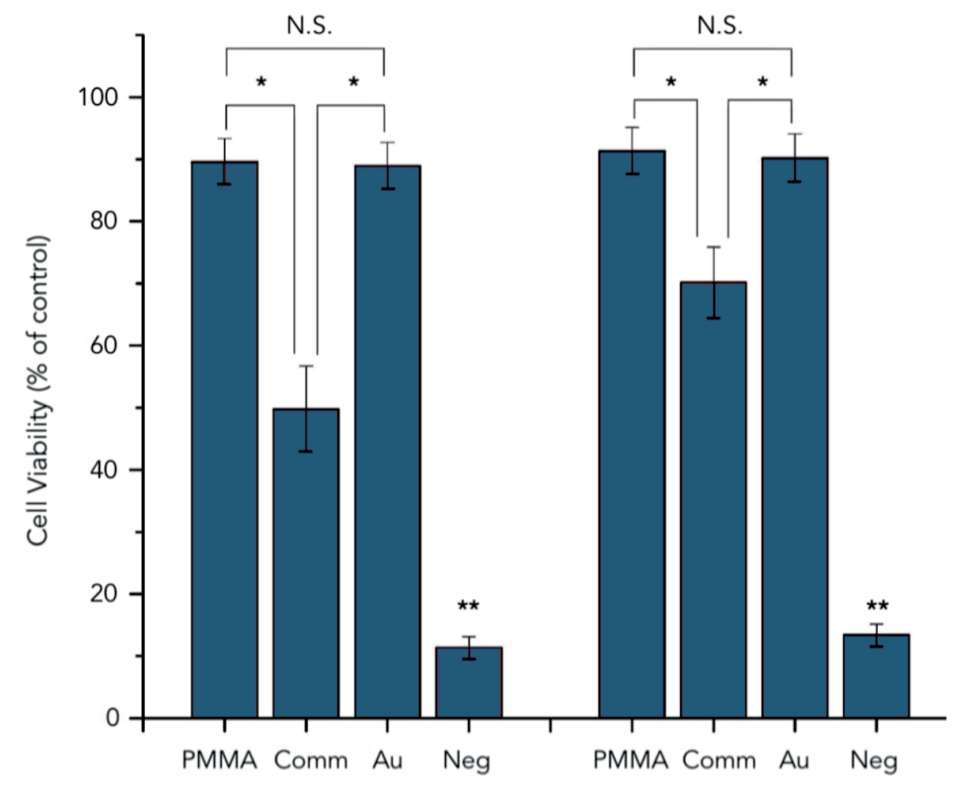

Figure 5. Cytotoxicity of the cements.

The level of toxicity of leachables is given in percentage of cell viability of the positive control. The bars represent the mean \pm standard deviation of 16 (negative control) to 32 (all cements) independent measurements. The ${ }^{*}$ represents significance between de commercial VertaPlex ${ }^{\mathrm{TM}}$ cement and the radiolucent (PMMA) and gold $(\mathrm{Au})$ cements $(\mathrm{P}<0.001)$. There was no significant difference between radiolucent and gold cements (N.S.). The negative control was significantly different from all cement samples (**; $\mathrm{P}<0.001)$. 


\subsubsection{Cytocompatibility of Cement}

In Figure 6 is shown the Live/Dead staining of L929 and SAOS-2 cells. The results were consistent for both cell types in these experiments; the large majority of the cells in all cement groups were viable with very few dead cells visible in each group. Green cells indicate viable cells, whereas red cells indicate dead cells due to the interaction between the ethidium-homodimer-1 and DNA. For the SAOS-2 cells, the whole cement surface is covered with viable cells. An almost complete coverage was obtained in three days, starting with a surface coverage of less than $10 \%\left(<30,000\right.$ cells $\left./ \mathrm{cm}^{2}\right)$. Fully confluent layers of these cells can reach up to 300,000 cells $/ \mathrm{cm}^{2}[23]$.

A.

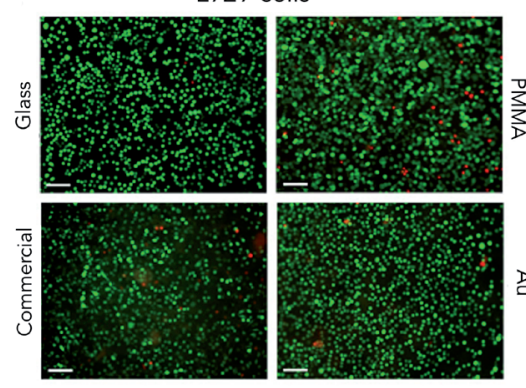

B.

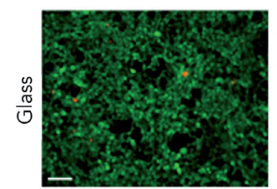

SAOS-2 cells

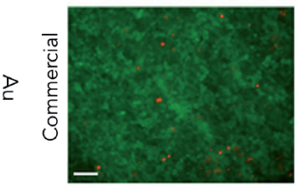

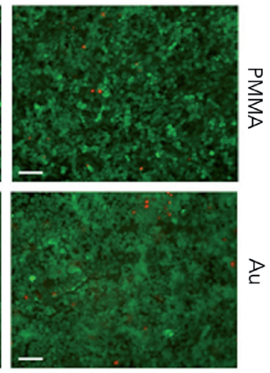

Figure 6. Live/Dead staining of A. L929 cells and B. SAOS-2 cells.

Green cells indicate viable cells, red indicates dead cells. The scale bars represent $100 \mu \mathrm{m}$.

To support these results, a series of cement samples with attached cells was prepared for analysis of cell morphology and confluency using SEM (Figure 7). For each of the cements, the L929 cells attached to the surface and spread. A number of dividing cells could also be observed on all surfaces. For the SAOS-2 cells, the confluent cell layers observed in the immunofluorescence experiments were also observed on the SEM images.

A.
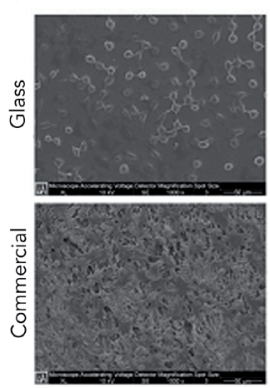

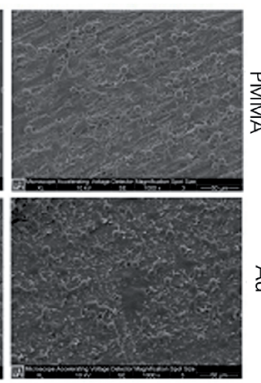

B.
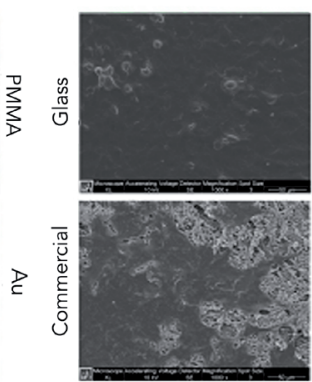

SAOS-2 cells

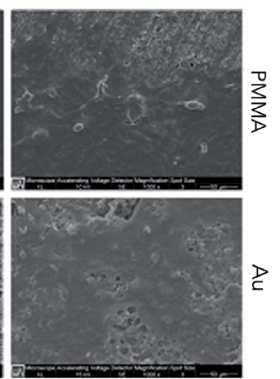

Figure 7. SEM images of cells, A. L929 fibroblasts and B. SAOS-2, on the different cements. The cells on glass cover slips are shown as control. 


\subsection{Biomechanical Testing}

\subsubsection{Compressive Properties of Cements}

The compressive modulus of the gold-containing cement was significantly greater than that of VertaPlex ${ }^{\mathrm{TM}}$, with the same trend seen for compressive strength (Table 1). Figure 1 shows a set of representative experimental stress-strain curves (including error bars).

Table 1. Summary of the compressive properties of the cements (mean \pm standard deviation).

\begin{tabular}{lcc}
\hline & $\begin{array}{c}\text { Compressive modulus } \\
(\mathbf{G P a})\end{array}$ & $\begin{array}{c}\text { Compressive strength } \\
(\mathbf{M P a})\end{array}$ \\
\hline VertaPlex $^{\mathrm{TM}}$ cement $(n=10)$ & $1.61 \pm 0.16^{*}$ & $52.73 \pm 4.53^{*}$ \\
Radiolucent cement $(n=8)$ & $2.50 \pm 0.22^{*}$ & $96.04 \pm 15.79^{*}$ \\
Gold-containing microsphere cement $(n=10)$ & $2.18 \pm 0.26$ & $80.88 \pm 10.57$ \\
\hline
\end{tabular}

*Values are significantly different from the gold cement $(\mathrm{p}<0.05)$

\subsubsection{Characteristics of the Simulated Osteoporotic Vertebral Compression}

\section{Fracture Model}

The definition of osteoporosis is defined as a T-score less than or equal to -2.5 SD; Based on the areal bone mineral density (aBMD) results the vertebral bodies used in are to be classified as "osteoporotic" (Table 2). The difference in aBMD and T-score between the two cadaveric spines was not significant (Table 2).

Table 2. Summary of the mean areal bone mineral density (aBMD) results for the cadaveric spines.

\begin{tabular}{llccc}
\hline Spine & Gender & Age (years) & aBMD $\left(\mathbf{g} / \mathbf{c m}^{2}\right)^{*}$ & T-score (SD) \\
\hline A & Female & 86 & 0.588 & $-4.2 \pm 0.8$ \\
B & Female & 91 & 0.579 & $-4.3 \pm 0.8$ \\
\hline
\end{tabular}

${ }^{*}$ No significant difference in aBMD between the spines $(\mathrm{p}=0.564)$

The volumetric BMD for the cortical and trabecular compartment of the vertebrae showed no significant differences between both groups (Table 3). Since there were no significant differences in volumetric BMD, and initial strength and stiffness between both experimental groups, adjacent vertebrae could be treated as paired specimens. There were no significant differences in either the initial compression strength or initial compression stiffness between vertebrae in the commercial cement group and the gold microsphere cement group paired specimens (Table 3).

There were no significant differences in either the post-augmentation compressive strength or the post-augmentation compressive stiffness between the VertaPlex ${ }^{\mathrm{TM}}$ cement group and the gold-containing microsphere cement group (Table 3). After cement 
augmentation, vertebral body strength increased significantly in the VertaPlex ${ }^{\mathrm{TM}}$ cement group and in the gold-containing cement group, relative to the initial strength value (Table 3). However, vertebral body stiffness was not restored to initial levels in each group and was even significantly less than initial stiffness (Table 3).

There were no significant differences in initial strength and stiffness or repaired stiffness between the lumbar and thoracic regions. However, although not significant, there was an observed trend towards a higher repaired strength in the thoracic regions for both experimental groups in comparison to the lumbar regions (Table 2).

Table 3. Summary of the vertebral body strength, vertebral body stiffness, injected cement volume, fill percentage, and density results (mean \pm standard deviation).

\begin{tabular}{|c|c|c|c|c|c|c|}
\hline \multirow[b]{2}{*}{ OVCF strength (N) } & \multicolumn{2}{|c|}{ Vertaplex $^{\mathrm{TM}}$ cement } & \multicolumn{2}{|c|}{$\begin{array}{c}\text { Gold-containing } \\
\text { microsphere cement }\end{array}$} & \multicolumn{2}{|c|}{ P-value } \\
\hline & Initial & Repaired & Initial & Repaired & Initial & Repaired \\
\hline Thoracic $(n=7)$, & $2366 \pm 1076$ & $4760 \pm 2103$ & $2176 \pm 612$ & $4402 \pm 2396$ & & \\
\hline Lumbar $(\mathrm{n}=4)^{*}$ & $2885 \pm 985$ & $4638 \pm 2491$ & $2742 \pm 462$ & $3143 \pm 1235$ & & \\
\hline Combined $(\mathrm{n}=11)$ & $2555 \pm 1027$ & $4716 \pm 2126 \sim$ & $2382 \pm 609$ & $3945 \pm 2075 \sim$ & 0.974 & 0.533 \\
\hline OVCF stiffness (N/mm) & Initial & Repaired & Initial & Repaired & Initial & Repaired \\
\hline Thoracic $(\mathrm{n}=7)$ ใ, & $1440 \pm 1079$ & $1127 \pm 350$ & $1163 \pm 532$ & $1170 \pm 823$ & & \\
\hline Lumbar $(\mathrm{n}=4)^{*}$ & $2519 \pm 800$ & $845 \pm 188$ & $2606 \pm 888$ & $1028 \pm 518$ & & \\
\hline Combined $(\mathrm{n}=11)$ & $1832 \pm 1089$ & $1024 \pm 323 \sim$ & $1688 \pm 967$ & $1119 \pm 702 \sim$ & 0.718 & 0.670 \\
\hline \multicolumn{7}{|c|}{ Injected cement volume (mL) } \\
\hline Thoracic $(\mathrm{n}=7)$ ใ, & \multicolumn{2}{|c|}{$5.57 \pm 1.17$} & \multicolumn{2}{|c|}{$5.50 \pm 1.12$} & & \\
\hline Lumbar $(n=4) *$ & \multicolumn{2}{|c|}{$6.50 \pm 1.08$} & \multicolumn{2}{|c|}{$7.50 \pm 0.58$} & & \\
\hline Combined $(\mathrm{n}=11)$ & \multicolumn{2}{|c|}{$5.91 \pm 1.18$} & \multicolumn{2}{|c|}{$6.23 \pm 1.37$} & \multicolumn{2}{|c|}{0.550} \\
\hline \multicolumn{7}{|l|}{ Fill percentage } \\
\hline Thoracic $(\mathrm{n}=7)$ ใ, & \multicolumn{2}{|c|}{$34 \% \pm 12 \%$} & \multicolumn{2}{|c|}{$29 \% \pm 14 \%$} & \multicolumn{2}{|c|}{0.654} \\
\hline Lumbar $(\mathrm{n}=4)^{*}$ & \multicolumn{2}{|c|}{$29 \% \pm 4 \%$} & \multicolumn{2}{|c|}{$27 \% \pm 6 \%$} & \multicolumn{2}{|c|}{0.081} \\
\hline Combined $(\mathrm{n}=11)$ & \multicolumn{2}{|c|}{$32 \% \pm 10 \%$} & \multicolumn{2}{|c|}{$28 \% \pm 11 \%$} & \multicolumn{2}{|c|}{0.532} \\
\hline \multicolumn{7}{|l|}{ Density (mg HA/cm³) } \\
\hline $\begin{array}{l}\text { Trabecular } \\
\text { compartment } q \text {, }\end{array}$ & \multicolumn{2}{|c|}{$75.18 \pm 27.11$} & \multicolumn{2}{|c|}{$70.59 \pm 25.24$} & \multicolumn{2}{|c|}{0.922} \\
\hline Cortical compartment ${ }^{*}$ & \multicolumn{2}{|c|}{$237.03 \pm 63.20$} & \multicolumn{2}{|c|}{$255.12 \pm 57.16$} & \multicolumn{2}{|c|}{0.974} \\
\hline Cement $\dagger$ & \multicolumn{2}{|c|}{$1878.21 \pm 79.95$} & \multicolumn{2}{|c|}{$1261.24 \pm 41.60$} & \multicolumn{2}{|c|}{0.000} \\
\hline
\end{tabular}

१, Values are not significantly different from each other in each group; ${ }^{*}$ Values are not significantly different from each other in each group; $†$ Significantly different between gold-containing microsphere cement and VertaPlex ${ }^{\mathrm{TM}}$ cement $(\mathrm{p}<0.05) ; \sim$ Significantly different from the initial value $(\mathrm{p}<0.05)$. 


\subsubsection{Cement Distributions and Radiopacity}

There was no significant difference in the volume of the cement injected between Verta$\mathrm{Plex}^{\mathrm{TM}}$ and the gold-containing cements in either spine region. For each cement group, fill percentage was lower, although not significantly so, for lumbar vertebrae compared to thoracic vertebrae (Figure 8; Table 3). The radiopacity of VertaPlex ${ }^{\mathrm{TM}}$ was significantly greater than that of the gold-containing cement.

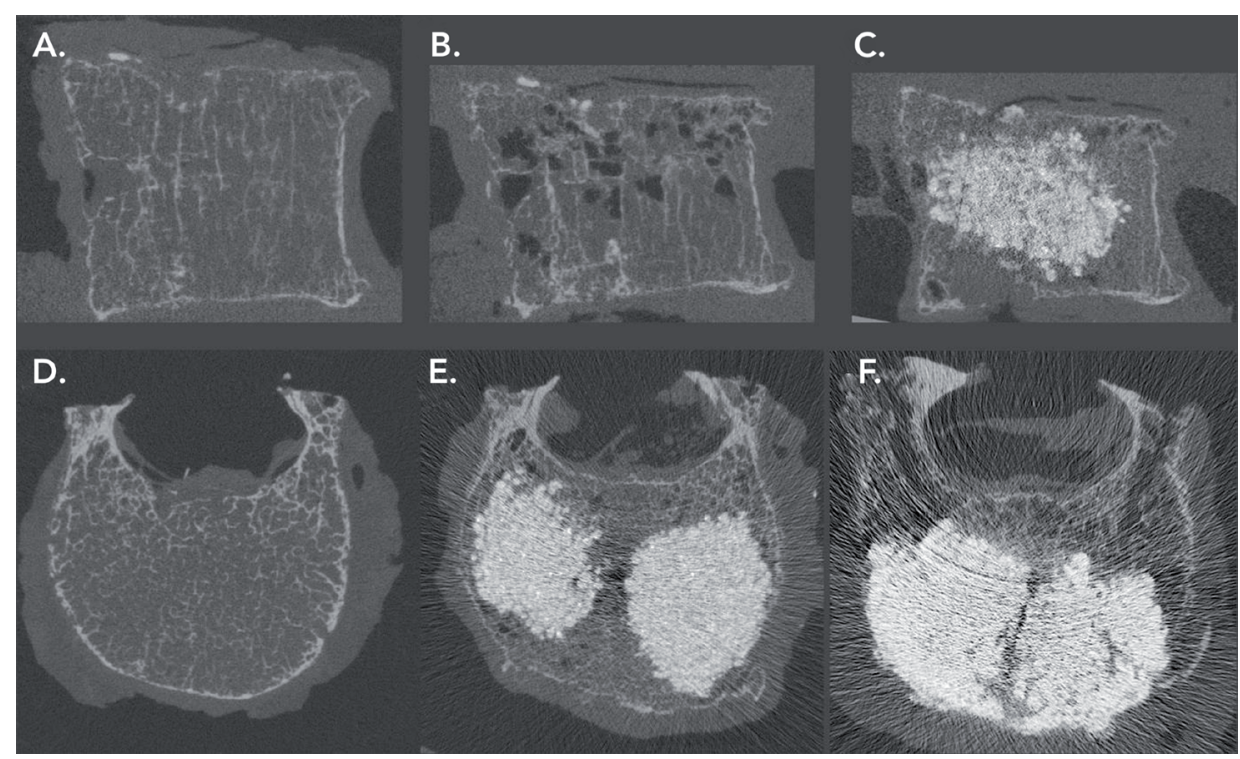

Figure 8. Axial and lateral HR-pQCT imaging of vertebral bodies before simulated compression fracture (A. and D.), with simulated compression fracture (B.) and after cement augmentation with either the novel gold-containing cement (C. and E.) or VertaPlex ${ }^{\mathrm{TM}}$ cement (F.).

\section{DISCUSSION}

The incorporation of barium sulphate $\left(\mathrm{BaSO}_{4}\right)$ as the radiopacifier in PMMA-based bone cements for percutaneous vertebroplasty has adverse effects on injectability, viscosity profile, setting time, mechanical properties of the cement and bone resorption [8-11]. In this study, we assessed a conceptually new type of cement comprising gold-containing microspheres. The first study purpose was to compare the novel cement and a counterpart commercial cement brand used for vertebroplasty in terms of in vitro injection time, cytocompatibility and mechanical properties. The second study purpose was to compare these two cements when used to augment fractured fresh-frozen cadaveric vertebral bodies by means of vertebroplasty on the basis of mechanical and filling properties and radiopacity. 
Most commercial acrylic bone cements are prepared with a mean PMMA particle size in the range of 10-60 $\mu \mathrm{m}$ [28]. In the present study, gold microspheres were used with a diameter varying between 0 and $300 \mu \mathrm{m}$. The larger particle size is likely responsible for the observed increase in curing properties of the cement, in terms of doughing time and injection time. Mixing and polymerization times can be optimized by altering size and size distribution of the PMMA microspheres $[12,28]$. The amount of initiator benzoyl peroxide (BPO) and/or the accelerator N,N-dimethyl-p-toluidine can also be modified. This means that an optimal composition can be obtained, optimizing the working time in order to allow the surgeon to inject the cement precisely. For this study, we chose to keep the formulation of the gold-containing microsphere and the radiolucent control cement the same as the VertaPlex ${ }^{\mathrm{TM}}$ cement leading to a cement formulation that needs a somewhat longer mixing time, but provides a larger window of injection time.

The elastic modulus and compressive strength for most commercial bone cements is reported to range between $1700-3700 \mathrm{MPa}$ and 85-114 MPa, respectively $[8,29,30]$. The compressive properties we measured for the gold-containing microsphere cement (2018 $\mathrm{MPa}$ and $81 \mathrm{MPa}$, respectively) are in general agreement with the previously published literature $[8,29,30]$. Although, curing conditions were the same for all cements and according to the manufacturer's specifications for the VertaPlex ${ }^{\mathrm{TM}}$ cement, the ultimate strength and elastic modulus of the VertaPlex ${ }^{\mathrm{TM}}$ cement that we found were outside the range reported in literature $[8,29,30]$. We could not find a clear explanation for this observed difference.

The gold-containing microsphere cement demonstrated an improved cytocompatibility as compared to the VertaPlex ${ }^{\mathrm{TM}}$ control cement that contains $\mathrm{BaSO}_{4}$. $\mathrm{BaSO}_{4}$ in the cement matrix is the most plausible candidate for leaching and causing the observed toxicity. Since in the novel bone cement radiopacity is attained by gold particles that are solidly incorporated in the microspheres, the likelihood of leaching out is substantially decreased. Additionally, gold particles are reported to be inert [22]. Additional osteolysis after the injection of gold-containing microsphere cement would therefore presumably be lower than for the commercial VertaPlex ${ }^{\mathrm{TM}}$ cement and appears to be caused by the PMMA matrix itself (Figure 5). However, the long term in vivo effects are beyond the scope of this paper.

Biomechanical evaluation in a simulated osteoporotic vertebral compression fracture model indicated that the ex vivo mechanical and filling properties are comparable for the gold-containing microsphere cement and the VertaPlex ${ }^{\mathrm{TM}}$ cement. For both cements, the post-augmentation strength was increased, while post-augmentation stiffness was decreased in comparison to the initial pre-augmentation values. These findings are consistent with those in other studies, in which similar cement volumes were used $[25,26,31,32]$. There is a clear correlation between fill percentage and mechanical properties after augmentation [31]. The observed differences in compressive property results between the lumbar and thoracic regions are related to both vertebral body size and the 
injected cement volume. Due to the smaller size, the fill percentages were higher for thoracic vertebrae than for lumbar vertebrae. However, the ideal strength and stiffness values required for fracture stabilization and pain relief remain unknown; as well as to what extent restoration of strength and stiffness actually contributes to clinical pain relief [31].

High radiopacity for good visibility on fluoroscopy is an absolute pre-requisite for injectable bone cements in the spine. Gold $(\mathrm{Z}=79)$ is a heavier element than barium $(\mathrm{Z}=56)$ which causes gold to have an almost three times higher attenuation coefficient than barium (250.8 1/cm vs. $86.01 / \mathrm{cm}$, respectively) [33]. Because of the higher attenuation coefficient of gold, the required concentration of gold in the cement is lower than the concentration of barium in the VertaPlex ${ }^{\mathrm{TM}}$ cement in order to obtain similar radiopacity. The concentration of gold in the novel cement lead to lower radiopacity than that of the Verteplex ${ }^{\mathrm{TM}}$ cement. Nevertheless, the cement was clearly visible (Figure $8 \mathrm{C}$ and $8 \mathrm{E}$ ) allowing for highly controllable and safe cement augmentation because the apparent density of the gold-containing microsphere cement was approximately five-fold higher when compared to the density of cortical bone. These values have been reported to be sufficient for successful injection into the fractured vertebra under continuous fluoroscopic guidance during vertebroplasty [34]. Additionally, higher concentrations of gold may lead to unwanted images artifacts on X-ray and Computed Tomography and would make it impossible to visualize the trabecular structure (as is the case for the VertaPlex ${ }^{\mathrm{TM}}$ cement, Figure 8F).

The limitations of the study can be summarized as follows: (1) The viscosity-versusmixing time profile of the experimental cement has not been characterized and optimized completely; (2) Extent and pattern of extravasation of the experimental cement were not fully characterized; (3) Fatigue life of fractured and augmented vertebral bodies were not determined; (4) Effects of different concentrations of gold in the cement were not examined; (5) Optimal size and size-distribution of the microspheres were not determined.

Regarding (4), it must be noted that increasing the gold content in the cement would not change the biocompatibility of the material, since the gold particles are embedded in the PMMA microspheres, which precludes any contact between the contrast medium and surrounding tissues. Furthermore, gold is noble (non-corrosive) and inert. On the other hand, cost is the obvious reason for keeping the gold content of the cement as low as possible. The optimum gold concentration still remains to be determined. Perhaps the most essential message emerging from this work is that tuning the X-ray contrast of the experimental cement is possible without running into problems of contrast leaching and inflammatory responses (as is the case for the $\mathrm{BaSO}_{4}$-containing counterpart cements for vertebroplasty). This is a significant advantage of the gold-containing cement. As in clinical practice, the cement injection was stopped if pending posterior extravasation was observed on fluoroscopy. Therefore, the fact that similar fill percentages were found 
for both cement groups indicates comparable cement filling and low leakage rate for the novel gold microsphere cement. Whereas the flow characteristics of the cement dough appeared favorable, the rheological and hardening behavior of the newly developed cement for the use in vertebroplasty warrants further investigation, and the optimal composition of the gold microsphere cement still has to be determined. Furthermore, due to the high decomposition rate of the cadaveric specimens at ambient temperature, it was impossible to test compressive fatigue life of fractured and augmented vertebral bodies. Finally, we wish to point out that a possible shortcoming of our study is that we used VertaPlex $^{\mathrm{TM}}$ rather than VertaPlex-HV as the reference material. Using VertaPlex-HV would have given a better match of the viscosity-versus mixing time profiles with a lower potential for cement extravasation during vertebroplasty.

In our opinion, a small-sized clinical pilot study would be the next step in order to perform an in vivo head-to-head comparison of the new gold-containing cement versus a commercial $\mathrm{BaSO}_{4}$-containing counterpart. Unfortunately, there is no mid-size or large animal model with osteoporotic vertebrae and the introduction of a compression fracture in an in vivo animal model would be highly unethical. Furthermore, extensive follow-up times would be required as the hypothesized advantage of the gold-containing cement (no leakage of contrast and, hence, less osteolysis) will be manifest only after years, and there is no reliable technique to monitor possible osteolysis around the cement in a non-invasive longitudinal manner. Moreover, studying biocompatibility of the new gold-containing cement in vivo, using small animal models, would merely show the well-known tissue reactions to PMMA cement, since the gold particles are completely inert, and they are not in contact with the surrounding tissues.

The results of the current study strongly suggest that the novel gold-containing microsphere cement may be an interesting modifiable alternative to the commercial PMMA bone cements. The gold microsphere cement featured excellent cell compatibility in vitro, as well as sufficient radiopacity in a realistic experimental set-up. Moreover, the cement provided mechanical stabilization and uniform filling similar to commercial bone cements. Further work to examine the optimal composition and the potential utility of the new cement is currently ongoing in our laboratories.

\section{CONCLUSION}

The results of the current study show that incorporation of gold particles in PMMA microspheres as radiopacifier improves the handling properties of the cement. In comparison with commercial VertaPlex ${ }^{\mathrm{TM}}$, the experimental gold-containing microsphere cement is superior in terms of cytocompatibility, Moreover, the compressive properties of the experimental cement are in general agreement with the previously published literature. Cement injection into human cadaveric vertebrae with simulated compres- 
sion fractures revealed the mechanical and filling properties of the gold-containing microsphere cement were not compromised. The gold-containing microsphere cement could be an interesting alternative to the commercial bone cements in the treatment of vertebral compression fractures. 


\section{REFERENCES}

1. Johnell O, Kanis JA. An estimate of worldwide prevalence and disability associated with osteoporotic fractures. Osteoporos Int 2006; 17: 1726-33.

2. Kim MJ, Lindsey DP, Hannibal M, Alamin TF. Vertebroplasty versus kyphoplasty: biomechanical behavior under repetitive loading conditions. Spine 2006; 31(18): 2079-84.

3. Jay B, Ahn SH. Vertebroplasty. Semin Intervent Radiol 2013; 30(3): 297-306.

4. Klazen CA, Lohle PN, de Vries J, Jansen FH, Tielbeek AV, Blonk MC, Venmans A, van Rooij WJ, Schoemaker MC, Juttmann JR, et al. Vertebroplasty versus conservative treatment in acute osteoporotic vertebral compression fractures (Vertos II): an open-label randomized trial. Lancet 2010; 376: 1085-92.

5. Lewis G. Viscoelastic properties of injectable bone cements for orthopaedic applications: state-of-the-art review. J Biomed Mater Res B: Appl Biomater 2011; 98(1): 171-91.

6. Lewis G. Percutaneous vertebroplasty and kyphoplasty for the stand-alone augmentation of osteoporosis-induced vertebral compression fractures: present status and future directions. J Biomed Mater Res B: Appl Biomater 2007; 81(2): 371-86.

7. Lewis G. Injectable bone cements for use in vertebroplasty and kyphoplasty: state-of-the-art review. J Biomed Mater Res B: Appl Biomater 2006; 76(2) :456-68.

8. Kürtz SM, Villaragga ML, Zhao K, Edidin AA. Static and fatigue mechanical behavior of bone cement with elevated barium sulphate content for treatment of vertebral compression fractures. Biomaterials 2005; 26(17): 3699-712.

9. Sabokbar A, Fujikawa Y, Murray DW, Athanasou NA. Radio-opaque agents in bone cement increase bone resorption. J Bone Joint Surg 1997; 74: 652-58.

10. Sabokbar A, Panday R, Quinn J, Athanasou NA. Osteoclastic differentiation by mononuclear phagocytes containing biomaterial particles. Arch Orthop Traum Surg 1998; 184: 31-36.

11. Wimhurst J, Brooks R, Rushton N. The effects of particulate bone cement at the bone-implant interface. J Bone Joint Surg 2001; 83: 588-92.

12. Rodrigues DC, Gilbert JL, Hasenwinkel JM. Pseudoplasticity and setting properties of twosolution bone cement containing poly(methyl methacrylate) microspheres and nanospheres for kyphoplasty and vertebroplasty. J Biomed Mater Res Part B: Appl Biomater 2009; 91B: 248-56.

13. Nieuwenhuijse MJ, van Erkel AR, Dijkstra S. Cement leakage in percutaneous vertebroplasty for osteoporotic vertebral compression fractures: identification of risk factors. The Spine Journal 2001; 11: 839-48.

14. Lewis G, van Hooy-Corstjens CSJ, Bhattaram A, Koole LH. Influence of the radiopacifier in an acrylic bone cement on its mechanical, thermal, and physical properties: barium sulphate containing cement versus iodine-containing cement. J Biomed Mater Res Part B: Appl Biomater 2005; 73B: 77-87.

15. Pepiol A, Teixidor F, Saralidze K, van der Marel C, Willems P, Voss L, Knetsch MLW, Vinas C, Koole LH. A highly radiopaque vertebroplasty cement using tetraiodinated o-carborane additive. Biomaterials 2011; 32: 6389-98.

16. Artola A, Gurruchaga M, Vázquez B, San Román J, Goñi I. Elimination of barium sulphate from acrylic bone cements. Use of two iodine-containing monomers. Biomaterials 2003; 24: 4071-80. 
17. Boelen EJ, Lewis G, Xu J, Slots T, Koole LH, van Hooy-Corstjens CS. Evaluation of a highlyradiopaque iodine-containing acrylic bone cement for use in augmentation of vertebral compression fractures. J Biomed Mater Res A 2008; 86(1): 76-88.

18. O'Brien D, Boyd D, Madigan S, Murphy S. Evaluation of a novel radiopacifiying agent on the physical properties of surgical spineplex. J Mater Sci: Mater Med 2010; 21: 53-8.

19. Hernández L, Fernández M, Collía F, Gurruchaga M, Goñi I. Preparation of acrylic bone cements for vertebroplasty with bismuth salicylate as radiopaque agent. Biomaterials 2006; 27 : 100-7.

20. Vega de la JC, Häfeli UO. Utilization of nanoparticles as X-ray contrast agents for diagnostic imaging applications. Contrast Media Mol. Imaging 2015; 10 (2): 81-95.

21. Camilleri J, Gandolfi MG. Evaluation of the radiopacity of calcium silicate cements containing different radiopacifiers. International Endodontic Journal 2010; 43: 21-30.

22. Shah M, Badwaik VD, Dakshinamurthy R. Biological applications of gold nanoparticles. J Nanosci Nanotechnol 2014; 14(1): 344-62.

23. Rodan SB, Imai Y, Thiede MA, Wesolowski G, Thompson D, Bar-Shavit Z, et al. Characterization of a human osteosarcoma cell line (SAOS-2) with osteoblastic properties. J Cancer Res 1987; 47: 4961-66.

24. Braet F, de Zanger R, Wisse E. Drying cells for SEM, AFM and TEM by hexamethyldisilazane: a study on hepatic endothelial cells. J Microsc 1997; 186, 84-87.

25. Tomita S, Molloy S, Jasper LE, Abe M, Belkoff SM. Biomechanical comparison of kyphoplasty with different bone cements. Spine 2004; 11: 1203-7.

26. Belkoff SM, Mathis JM, Deramond H, et al. An ex vivo biomechanical evaluation of a hydroxyapatite cement for use with kyphoplasty. AJNR Am J Neuroradiol 2001; 22: 1212-16.

27. Kinzl M, Benneker LM, Boger A, Zysset PK, Pahr DH. The effect of standard and low-modulus cement augmentation on the stiffness, strength, and endplate pressure distribution in vertebroplasty. Eur Spine J 2012; 21: 920-29.

28. Pascual B, Vázquez B, Gurruchaga M, Goñi I, Ginebra MP, Gil FJ, Planell JA, Levenfeld B, San Roman J. New aspects of the effect of size and size distribution on the setting parameters and mechanical properties of acrylic bone cements. Biomaterials 1996; 17: 509-16.

29. Hernandez L, Muñoz ME, Goñi I, Gurruchaga M. New injectable and radiopaque antibiotic loaded acrylic bone cements. J Biomed Mater Res B: Appl Biomater 2008; 87B(2): 312-20.

30. American Society for Testing Materials (ASTM). ASTM Standard F451-99a: Standard specification for acrylic bone cement. In Annual Book of ASTM Standards. Vol 13.01. ASTM, West Conshohocken, PA, United States.

31. Belkoff SM, Mathis JM, Jasper LE, Deramond H. The biomechanics of vertebroplasty: the effect of cement volume on mechanical behavior. Spine 2001; 14: 1537-41.

32. Belkoff SM, Mathis JM, Jasper LE. Ex vivo biomechanical comparison of hydroxyapatite and polymethylmethacrylate cements for use with vertebroplasty. AJNR Am J Neuroradiol 2002; 23: $1647-51$.

33. Tables of X-Ray Mass Attenuation Coefficients and Mass Energy-Absorption Coefficients (version 1.4) [database on the Internet]. National Institute of Standards and Technology, Gaithersburg, MD. 2004. Available from: http://physics.nist.gov/xaamdi.

34. Lu Y, Krause M, Bishop N, Sellenschloh K, Glüer CC, Püschel K, Amling M, Morlock MM, Huber G. The role of patient-mode high-resolution peripheral quantitative computed tomography indices in the prediction of failure strength of the elderly women's thoracic vertebral body. Osteoporos Int 2015; 26: 237-44. 



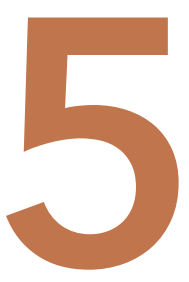

Reduction of intradiscal pressure by the use of polycarbonate-urethane rods as compared to titanium rods in posterior thoracolumbar spinal fixation

Jacobs E, Roth AK, Arts JJ, van Rhijn LW, Willems PC 


\section{ABSTRACT}

Loss of sagittal alignment and balance in adult spinal deformity can cause severe pain, disability and progressive neurological deficit. When conservative treatment has failed, spinal fusion using rigid instrumentation is currently the salvage treatment to stop further curve progression. However, fusion surgery is associated with high revision rates due to instrumentation failure and proximal junctional failure, especially if patients also suffer from osteoporosis. To address these drawbacks, a less rigid rod construct is proposed, which is hypothesized to provide a more gradual transition of force and load distribution over spinal segments in comparison to stiff titanium rods. In this study, the effect of variation in rod stiffness on the intradiscal pressure (IDP) of fixed spinal segments during flexion-compression loading was assessed. An ex vivo multisegment (porcine) flexion-compression spine test comparing rigid titanium rods with more flexible polycarbonate-urethane (PCU) rods was used. An increase in peak IDP was found for both the titanium and PCU instrumentation groups as compared to the uninstrumented controls. The peak IDP for the spines instrumented with the PCU rods was significantly lower in comparison to the titanium instrumentation group. These results demonstrated the differences in mechanical load transfer characteristics between PCU and titanium rod constructs when subjected to flexion-compression loading. The concept of stabilization with a less rigid rod may be an alternative to fusion with rigid instrumentation, with the aim of decreasing mechanical stress on the instrumented segments and the possible benefit of a decrease in the incidence of screw pullout. 


\section{INTRODUCTION}

Vertebral body wedging may be the result of an anterior vertebral compression fracture or gradual deformation due to creep-like mechanisms [1]. Subsequent spinal deformity is often progressive and wedge accumulation over multiple thoracolumbar levels may lead to thoracolumbar hyperkyphosis. Factors such as (asymmetric) intervertebral disc degeneration, reduced spinal muscle strength, and weakening of the spinal ligament support also contribute to progressive thoracolumbar curvature [2,3]. Inability to compensate for thoracolumbar hyperkyphosis leads to global spinal sagittal malalignment, which is the strongest driver of pain and disability $[2,4,5]$.

Operative correction is not typically recommended as the first treatment option due to the high prevalence of osteoporosis and other medical comorbidities in the elderly patient population $[2,6,7]$. Instrumented spinal fusion is regarded more as an end-stage salvage procedure only reserved for patients suffering from intractable pain, severe disability, significant pulmonary function impairment or progressive neurological deficit not amenable to conservative treatment [3, 6-9]. This implies that patients considered for surgery are in an advanced stage of the degenerative process, with frequently compromised bone quality due to osteoporosis or osteopenia and/or severe global sagittal imbalance. As a result, surgery in these patients is associated with high rates of complications, such as proximal junctional failure (PJF), instrumentation breakout and recurrent spinal deformity $[6,8,10]$. In patients older than 55 years the risk of PJF is close to $40 \%$, and as many as one-third of the patients undergo early revision within one year [6].

Bone quality is an important factor that influences stability of posterior spinal implants. Posterior instrumentation tends to fail due to limited screw-bone interface fixation strength in low-density bone, resulting in screw pullout, transitional fractures and loosening [11, 12]. Ohtori et al. [13] found that the incidence of screw pullout in the osteoporotic spine after 1- or 2-level posterolateral fusion for degenerative spondylolisthesis ranges from $15 \%$ to $25 \%$. Conversely, the occurrence in young patients suffering from Scheuermann's disease is relatively low and ranges from $0 \%$ to $3 \%$ [14]. Mechanical stiffness mismatch between rigid spinal instrumentation and osteoporotic bone contributes to the increased incidence of failure in the surgical treatment of hyperkyphosis in the elderly $[2,6,14,15]$.

The concept of "semirigid" fixation has been introduced as an alternative to rigid metal constructs in order to minimize instrumentation related problems [14, 16-18]. A semirigid stabilization system would promote fusion via a balance of stability and intra- and inter-level load sharing [16, 19-21]. Polyetheretherketone (PEEK) rods have been proposed as such, as the Young's modulus of PEEK is approximately 30-fold lower compared to Titanium (3.5 vs. 115 GPa, respectively) [22, 23]. Chou et al. [23] compared a PEEK rods construct to a titanium rods construct and found in PEEK rods a loading pattern more comparable to those of the intact spine based on the biomechanical changes of 
the disc height and IDP at both the instrumented and the adjacent levels. Moreover, rod stresses and bone stresses at the screw-bone interface measured by means of uniaxial strain gauges were reduced for the PEEK rod constructs, indicating a reduced risk of instrumentation failure [23].

Schmidt et al. [24] used finite element analysis to calculate the ideal axial and bending stiffness of a posterior stabilization device for different degrees of segmental stabilization. For semirigid fixation, defined as a reduction in range of motion up to $33 \%$, the ideal axial stiffness was defined as approximately $45 \mathrm{~N} / \mathrm{mm}$, calculated as:

$$
c(a)=\frac{E A}{l}
$$

where $l$ is the length, A the area and $E$ the Young's modulus. The ideal bending stiffness was defined as approximately $30 \mathrm{~N} / \mathrm{mm}$ and calculated as:

$$
\mathrm{c}(b)=\frac{3 \mathrm{E} I}{l^{2}}
$$

where $I$ is the second moment of area [24]. Using PEEK as a material results in substantially higher stiffness values as required for semirigid fixation (axial stiffness $1386 \mathrm{~N} /$ mm, bending stiffness $131 \mathrm{~N} / \mathrm{mm}$ ) [23, 24]. This has been confirmed by Rohlmann et al. [25], who showed that only very low axial stiffness $(<200 \mathrm{~N} / \mathrm{mm})$ of a posterior rod influences the segment kinematics. Therefore, we propose using a material with a much lower Young's modulus for the early stage stabilization of (impending) thoracolumbar hyperkyphosis: polycarbonate-urethane (PCU). Polyurethane elastomers are typically composed of three reactive components: a diisocyanate, an oligomeric macromonomer and a chain extender [26], which can be combined in different ratios to produce polyurethane elastomers with vastly differing physicochemical and mechanical properties. The shore hardness of PCU is temperature dependent and reduces as temperature rises. The purpose of this study is to comparatively analyze the effect of spinal rod stiffness variation on intradiscal pressure (IDP) by means of PCU and titanium rod constructs subjected to dynamic flexion-compression loading. We hypothesize that a more flexible construct will result in reduced spinal load (intradiscal pressure). As reduced spinal load has been associated with reduced stresses at the screw-bone interface, the risk of instrumentation failure and PJF may thus potentially be reduced. 


\section{MATERIALS AND METHODS}

\subsection{Specimen Preparation}

Twenty porcine lumbar spine specimens were obtained from a local abattoir. At time of slaughter, all female animals weighed between 80 and 90 kilograms (10-14 months old). The spines were dissected and sectioned into multi-level segments from L1 to L5. All soft tissue was carefully removed leaving the ligaments, intervertebral discs and facet joint capsules intact. Radiographs of the porcine spines were made to exclude any anatomic abnormalities. The porcine specimens used in the current study showed normal spine morphology without radiologic evidence of any spinal pathology. After preparation, the specimens were wrapped in saline soaked gauze, sealed in plastic bags and stored at $-18^{\circ} \mathrm{C}$. The segments were thawed in a $4^{\circ} \mathrm{C}$ environment to acclimate to room temperature approximately 24 hours prior to testing. The porcine cadaveric specimens underwent only one freeze-thaw cycle, which according to the literature has no negative effect on the biomechanical properties of the spine [27-29]. The upper and lower half of respectively vertebrae L1 and L5 were both fixed in cups fitting the test setup (Figure 1), using polymethylmethacrylate (PMMA, Technovit 3040, Heraeus, Hanau, Germany). Screws were driven into the endplates of the cranial (L1) and caudal (L5) vertebrae to improve fixation between the PMMA and the vertebrae.

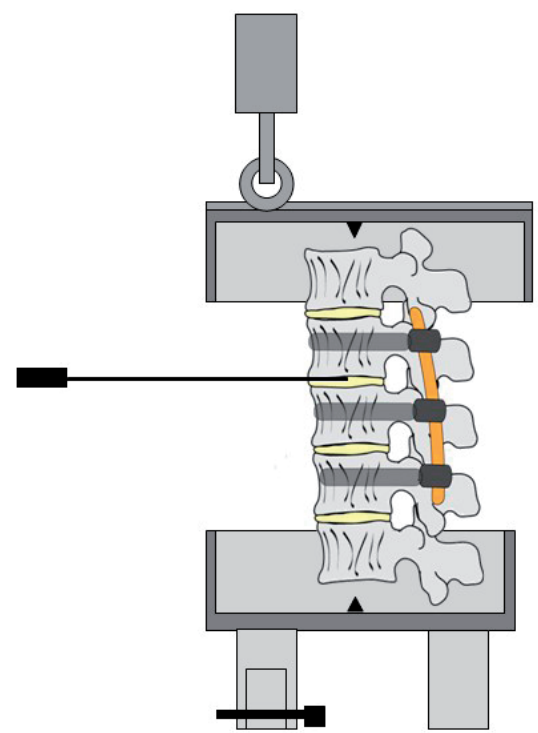

Figure 1. Schematic of the custom designed flexion-compression test set-up.

The lower metal cup was firmly attached to the materials testing machine. The spinal segment was fixated by means of PMMA to the lower and upper metal cup. A roller was mounted on the vertical actuator of the materials testing machine and was forced to traverse the slot in the upper cup introducing a flexion movement of the spine. A counter balance mechanism was applied to return the spinal segment to its neutral position. 


\subsection{Specimen Instrumentation}

Four different groups were tested: (1) uninstrumented spines $(n=5)$, pedicle screw instrumentation with (2) titanium rod fixation (n=5), (3) PCU (Bionate ${ }^{\circledast}$, DSM Biomedical, Geleen, the Netherlands) shore hardness 75D rod fixation ( $\mathrm{n}=5)$, and (4) PCU shore Hardness 65D rod fixation $(n=5)$. The Young's modulus of the different rods was $115,000 \mathrm{~N} /$ $\mathrm{mm}^{2}$ for the titanium rods, $188 \mathrm{~N} / \mathrm{mm}^{2}$ for the 75D PCU rods and $120 \mathrm{~N} / \mathrm{mm}^{2}$ for the 65 PCU rods at $37^{\circ} \mathrm{C}$. The PCU rods were rehydrated in a saline bath at $37^{\circ} \mathrm{C}$ for at least 48 hours prior to implantation. Posterior instrumentation spanning three motion segments was applied to fifteen fresh porcine spinal segments (L2, L3 and L4) by use of standard surgical technique. Six mono-axial titanium alloy pedicle screws (30-mm length, 5.5-mm diameter, Medtronic Inc., Minneapolis, MN) were inserted transpedicularly without wall breach under fluoroscopic guidance. Plain anteroposterior and lateral radiographs were taken to confirm correct placement of the pedicle screws and to exclude bone abnormalities. Screw-rod instrumentation was applied to the L2 to L4 segment consisting of the aforementioned six pedicle screws and either the PCU or titanium alloy rods, all $120 \mathrm{~mm}$ in length and $5.5 \mathrm{~mm}$ in diameter. The use of PCU rods with these dimensions allowed for a fundamental comparison. Table 1 shows an overview of the material properties for the different rods used, including the length, diameter, Young's modulus, axial stiffness and bending stiffness.

Table 1. Overview of the material properties of the rods (mean \pm standard deviation).

\begin{tabular}{|c|c|c|c|c|c|}
\hline & $\begin{array}{l}\text { Length } \\
(\mathrm{cm})\end{array}$ & $\begin{array}{l}\text { Diameter } \\
(\mathbf{m m})\end{array}$ & $\begin{array}{l}\text { Young's modulus } \\
\left(\mathrm{N} / \mathrm{mm}^{2}\right)\end{array}$ & $\begin{array}{l}\text { Axial stiffness } \\
(\mathbf{N} / \mathbf{m m})\end{array}$ & $\begin{array}{c}\text { Bending stiffness } \\
(\mathrm{N} / \mathbf{m m})\end{array}$ \\
\hline Titanium & 12 & 5.5 & 115,000 & 22,768 & 1076 \\
\hline PCU 75D & 12 & 5.5 & $188 \pm 30$ & 37 & 2 \\
\hline PCU 65D & 12 & 5.5 & $120 \pm 20$ & 24 & 1 \\
\hline
\end{tabular}

\subsection{Mechanical Testing}

A combined flexion-compression load was applied to the spinal segments by means of an eccentric force [30]. The tests were conducted in a custom designed test setup placed in a saline bath at $37^{\circ} \mathrm{C}$ in order to mimic body temperature and to account for the temperature dependent material properties of the PCU rod (Figure 1). The lower metal cup was firmly attached to the materials testing machine (Zwick/Roell, ZMART PRO) after fixation of the spinal segment in PMMA. A roller was mounted on the vertical actuator of the materials testing machine containing a load cell. When the load was applied, the roller was forced to traverse the slot in the upper cup in the anterior-posterior direction, thereby preventing out-of-plane bending. A counter balance mechanism was applied in order to return the spinal segment to its neutral position. The vertical actuator applied a $115 \mathrm{~N}$ load, 65-mm anterior to the midpoint of the L1 vertebral body, without preload, 
creating flexion combined with axial compression of the specimen [27]. The load was applied with a vertical actuator speed of $400-\mathrm{mm} / \mathrm{min}$ for a total of 3.000 cycles.

\subsection{Intradiscal Pressure Measurements}

A 1.3-mm diameter pressure transducer $(8 \mathrm{CT} / 4 \mathrm{~F} / \mathrm{SS} / \mathrm{HP}$, Gaeltec Ltd, linearity error $<1 \pm$ $1 \%$ ) was used to measure IDP. The annulus was punctured using an 18 gauge needle, and the transducer was inserted into the L2-L3 disc before testing. Continuous measurement was performed.

During loading of the spinal segment, IDP was measured and the average results were calculated after $0,100,250,500,1.000,1.500,2.000,2.500$, and 3.000 loading cycles, respectively.

\subsection{Statistical Analysis}

Statistical analysis was performed on IDP data using SPSS version 20 for Windows (SPSS, Inc., Chicago, IL). Mean data obtained during sagittal plane testing were compared between intact and different instrumented testing using a Mann-Whitney U test (significance level at $\mathrm{p}<0.05)$. Since this study is a concept study a post-hoc power analysis was performed.

\section{RESULTS}

Stabilization of the viscoelastic response of the spines occurred after approximately 1,500 cycles of loading for all testing conditions (Figure 2). Peak IDP at the L2-L3 level for all groups is shown in Table 2 and Figure 2.

The initial peak IDP, at 0 cycles, increased in the presence of instrumentation. The greatest significant increase in initial IDP as compared to the uninstrumented spines, was found in the titanium instrumented group ( 1.33 vs. $0.81 \mathrm{MPa}, \mathrm{p}=0.009$ ). This was not the case for the 75D and 65D PCU instrumented spines (0.86 and 0.59 MPa respectively vs. $0.81 \mathrm{MPa}, \mathrm{p}>0.05$ ). The uninstrumented and PCU instrumented spines consistently produced significantly lower IDPs throughout the test compared to the titanium instrumented spines $(\mathrm{p}<0.05)$. After 500 cycles the peak IDP also increased significantly for the 75D PCU instrumented spines in comparison to the uninstrumented spines $(\mathrm{p}<0.05)$.

After 3,000 cycles the IDP for spines instrumented with a titanium rod was significantly higher than that of all other groups $(\mathrm{p}=0.009)$. For the spines instrumented with a 75D PCU rod the IDP after 3,000 cycles was significantly higher in comparison to the spines instrumented with a 65D PCU rod or the uninstrumented spines ( 0.54 vs. 0.33 and 0.30 respectively, $\mathrm{p}=0.009$ ). There was no significant difference in IDP between the 65D PCU rod and the uninstrumented spines at 3,000 loading cycles $(\mathrm{p}=0.347)$. 


\section{L2-3 Intradiscal Pressure}

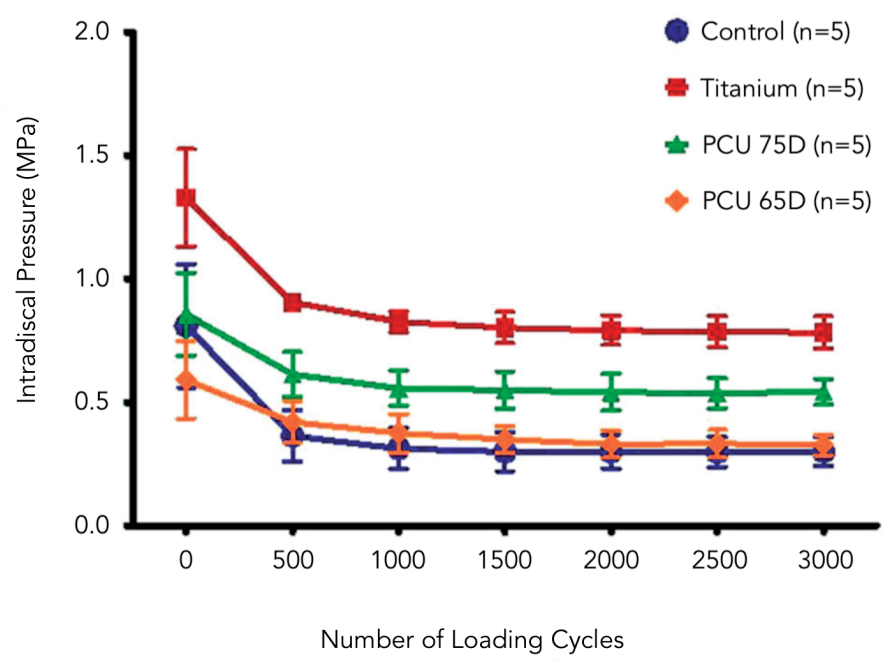

Figure 2. Mean L2-L3 intradiscal pressures (IDP) within instrumented segments L2-L3. The error bars represent the mean \pm standard deviation of 5 independent measurements for all three instrumented groups and the uninstrumented control group.

The results of the post-hoc power analysis indicated that the sample size of five spines per group is sufficient to find a significant difference between the different groups. Therefore the chosen sample size was determined to be adequate considering the objective of the concept study and the consistency in the biomechanical output.

Table 2. Summary of the intradiscal pressure results in MPa (mean \pm standard deviation) for the uninstrumented lumbar spines and the groups with titanium or PCU 65D and 75D instrumentation, respectively.

\begin{tabular}{lccccccc}
\hline & \multicolumn{7}{c}{ Number of loading cycles } \\
\cline { 2 - 7 } Group & $\mathbf{0}$ & $\mathbf{5 0 0}$ & $\mathbf{1 0 0 0}$ & $\mathbf{1 5 0 0}$ & $\mathbf{2 0 0 0}$ & $\mathbf{2 5 0 0}$ & $\mathbf{3 0 0 0}$ \\
\hline Control (n=5) & $0.81 \pm 0.25$ & $0.36 \pm 0.10$ & $0.31 \pm 0.08$ & $0.30 \pm 0.08$ & $0.30 \pm 0.07$ & $0.30 \pm 0.06$ & $0.30 \pm 0.06$ \\
Titanium (n=5) & $1.33 \pm 0.20^{*}$ & $0.90 \pm 0.02^{*}$ & $0.83 \pm 0.04^{*}$ & $0.80 \pm 0.06^{*}$ & $0.79 \pm 0.06^{*}$ & $0.79 \pm 0.06^{*}$ & $0.78 \pm 0.06^{*}$ \\
PCU 75D (n=5) & $0.86 \pm 0.17$ & $0.61 \pm 0.09^{* *}$ & $0.56 \pm 0.07^{* *}$ & $0.55 \pm 0.08^{* *}$ & $0.54 \pm 0.07^{* *}$ & $0.54 \pm 0.06^{* *}$ & $0.54 \pm 0.05^{* *}$ \\
PCU 65D (n=5) & $0.59 \pm 0.16$ & $0.42 \pm 0.09$ & $0.38 \pm 0.08$ & $0.35 \pm 0.05$ & $0.33 \pm 0.06$ & $0.33 \pm 0.06$ & $0.33 \pm 0.04$ \\
\hline
\end{tabular}

The "represents the significant difference between the titanium instrumented spines and the uninstrumented and PCU instrumented spines $(\mathrm{p}=0.009)$. The " represents the significant difference between the 75D PCU instrumented spines and the spines instrumented with a 65D PCU rod or the uninstrumented spines $(\mathrm{p}<0.05)$. 


\section{DISCUSSION}

Spinal fusion using long segment rigid instrumentation is currently the only surgical treatment to correct or stop curve progression in patients suffering from severe thoracolumbar hyperkyphosis $[2,3,7]$. Instrumentation failure or transitional fractures often occur as a result of diminished bone quality or sagittal imbalance of the patient, which leads to high rates of revision surgery $[6,8,14]$. Some of these complications can be attributed to high device stiffness. In this study, we assessed a conceptually new rod construct comprised of PCU with lower axial stiffness. The purpose was to obtain more fundamental insight into the effect of spinal rod stiffness variation on mechanical load transfer in the spine using PCU and titanium rods during cyclic flexion- compression loading.

The PCU rods construct demonstrated more comparable intradiscal pressure (IDP) outcomes to those of the intact spine when compared with the titanium rods construct, indicating a more physiological load distribution. The IDP, a direct measurement of mechanical load transfer through the anterior spinal column, is dependent on the range of motion of the spine [40]. The use of posterior instrumentation causes the center of rotation to move posteriorly beyond the posterior vertebral body line and superiorly into the disc space [21]. This posterior shift of the center of rotation causes axial compression and a higher loading of the intervertebral disc during flexion (i.e. higher IDP) [20, 31, 32]. Schilling et al. [20] studied the influence of design parameters of different pedicle screw based motion preservation systems on load transfer within the intervertebral disc and concluded that implant axial stiffness was the most important parameter. In their in vitro study low axial stiffness resulted in a lower amount of stabilization in the sagittal plane in comparison to stabilization provided by instrumentation with high axial stiffness [20]. This has been confirmed by Jahng et al. [21], whose finite element model showed that the shift in center of rotation differs according to the design and properties of the materials used for pedicle-based dynamic stabilization systems. The more rigid the pedicle-based dynamic stabilization device, the more the instantaneous center of rotation is shifted posteriorly and thus the nucleus pulposus is subjected to higher compressive loads. The interdependency between the axial stiffness of a posterior device on the amount of load transfer in the intervertebral disc is also confirmed by the significant difference in peak IDP between the 75D and 65D PCU instrumented groups in the current study. The axial stiffness of the 5.5-mm diameter rods used in the current study was calculated to be $22,768 \mathrm{~N} / \mathrm{mm}$ for titanium rods, $37 \mathrm{~N} / \mathrm{mm}$ for 75D PCU rods and $24 \mathrm{~N} / \mathrm{mm}$ for 65D PCU rods at $37^{\circ} \mathrm{C}$ according to equation 1 . The axial stiffnesses of the PCU rods are within the range of a semirigid implant defined by Schmidt et al. [26]. It is therefore expected that both PCU rods will reduce the spinal flexibility (range of motion) up to only $33 \%$ compared to the non-fixated situation, whereas the rigid titanium rod will provide a maximum reduction in spinal flexibility of $66 \%$ compared to the non-fixated situation. 
These results indicate that when using spinal implants with low stiffness there is still some degree of stabilization of the spine combined with a loading pattern more comparable with the intact, uninstrumented spine.

The bending stiffness of the rods used in the current study, as calculated according to equation 2, are approximately $1076 \mathrm{~N} / \mathrm{mm}$ for titanium rods, $2 \mathrm{~N} / \mathrm{mm}$ for 75D PCU rods and $1 \mathrm{~N} / \mathrm{mm}$ for $65 \mathrm{D}$ PCU rods at $37^{\circ} \mathrm{C}$. The bending stiffness of the PCU rods, in contrast to the axial stiffness, are outside the range of a semirigid rod as defined by Schmidt et al., being approximately 15 to 30 times lower [24]. In flexion and extension the effect of bending stiffness on the segmental stabilization is negligible, however, it is of high importance in lateral bending and axial rotation. According to the beam theory derived by Euler and Bernoulli, low bending stiffness increases the risk of buckling when an axial force is applied, since the critical force needed for buckling is proportional to the moment of inertia and the modulus of elasticity. By altering the geometry and/or elasticity of the rod the risk of buckling might be decreased. The PCU rods used in this study served the sole purpose of providing more fundamental insight on the effect of rod stiffness variation on the IDP. It still remains unclear which exact axial and bending stiffness and rod geometry will prove to be the most successful for less rigid stabilization with the optimal balance between attained correction and a minimal raise in load transfer through the instrumented spinal segments in patients suffering from thoracolumbar hyperkyphosis [20,24].

Chou et al. [23], compared the effect of PEEK versus titanium rod constructs on the disc height and IDP in the instrumented and adjacent levels after fatigue loading. A reduction of load transfer at the instrumented level subsequently resulted in an increased loading through the adjacent levels in titanium instrumented spines. PEEK rods were found to have a better capacity to reduce the non-physiological loadings at the cranial and caudal levels leading to a lower incidence of adjacent level failure [23]. The PCU rods used in the current study showed a comparable biomechanical pattern to the PEEK rods used by Chou et al. [23], and sustained a loading pattern more consistent with the uninstrumented spinal segment test group. This is of high clinical importance since pressures within normal physiological range are critical in providing a suitable environment to prevent instrument failure or proximal junctional failure (PJF) [33]. Based on the results of the current study it is expected that less rigid rods will allow better load sharing among spinal components leading to less junctional segment failure.

Mechanical stiffness mismatch between rigid spinal instrumentation and low mineral density bone has been suggested to contribute to high failure rates $[8,10,12]$.One of the goals of a more flexible rod is minimizing load transfer and subsequent stresses at the bone-screw interface. A similar pattern as found by Chou et al. [23], who confirmed that the stress on PEEK rods and the bone stresses near the bone-screw interface were significantly lower compared to titanium, is expected to apply for PCU rods [34]. However, Galbusera et al. [35] described a tendency to high screw loosening rates after stabilization 
with a less rigid rod in their literature review based on a study of Schatzker et al. [34]. This was explained by the absence of bony fusion leading to micromotion and formation of fibrous tissue around the screws [35, 36]. In our opinion, in order to circumvent this problem, screw augmentation with bone cement or fixation with laminar wires instead of pedicle screws could be used in conjunction with the less rigid PCU rods in the osteoporotic patient population [35, 37].

The present study has some limitations. First, only flexion-compression loading was tested in this study. This is due to the fact that hyperkyphosis is a single plane deformity in which the flexion movement of the spine is affected the most. In future research, a more advanced spinal loading simulator should be used which is capable of simulating flexion/extension moments, lateral bending moments and axial rotation [27]. Second, the global range of motion was not measured, which made analysis of standard momentrotation curves not possible [38]. The IDP provides a direct measurement of mechanical load transfer in the spine. Therefore, measurement of the full range of motion would be better to quantify the acute stabilization effects of posterior pedicle screw instrumentation in comparison to the uninstrumented spine. Third, the effect of rod stiffness variation on mechanical load transfer in the adjacent discs and stresses on the bone-screw interface were not assessed. Fourth, the inherent motion properties of the specimen were not measured before testing the stabilizing capacities of the implant systems. For future research this is necessary in order to assure that mechanically defective specimens are excluded and to provide an individual basis for normalization [27]. Fifth, the effect of stress relaxation and creep of the polymer components was not assessed. To this end, at least 180,000 cycles, representative for six weeks in vivo human movement, should be carried out [39]. In our study long-term cycling was impossible to test due to the high decomposition rate of the cadaveric specimens at $37^{\circ} \mathrm{C}$. However, Bionate ${ }^{\circledast}$ II PCU is currently being used in a wide range of applications, including neurostimulation, vascular, artificial heart, and in various orthopedic applications. It is one of the most extensively tested families of biomedical polymers and is backed by a comprehensive FDA master file. Bionate ${ }^{\circledast}$ II PCU is proven to be biostable and biocompatible. At last, quadrupeds have often been used for spinal research [40]. The porcine spine has, in specific situations, proven to be the most representative biomechanical model for the human spine [41, 42]. When spinal instrumentation is tested in the porcine, some general differences need to be kept in mind. The axial compression stress in quadrupeds is higher, which leads to higher bone mineral densities in the vertebrae. Due to the higher bone mineral density, the porcine bone is harder compared to the human osteoporotic bone. This results in a higher fixation strength for pedicle screws inserted into porcine vertebrae [40]. The porcine spine shows a less pronounced thoracic kyphosis and lumbar lordosis, compared to the human spine [42]. Moreover, the lumbar porcine spine is slightly kyphotic. The range of motion, particularly in flexion/extension, is over all levels much smaller compared to the human range of motion [43]. This is mainly due to the different orientation 
of the facet joints. The facet joint surfaces of the porcine spine are oriented at an angle of less than 30 degrees relative to the frontal plane compared to an angle of more than 60 degrees in the human spine [41, 42, 44]. Thoracolumbar hyperkyphosis mainly affects the thoracolumbar part of the human spine. However, the porcine thoracic region is not comparable to the human thoracic region due to differences in anatomical dimensions, facet joint orientation and length of spinous process. In the porcine, the anterioposterior vertebral depth and vertebral body width are smaller compared to the respective human dimensions. Dath et al. described the measurements for the width and depth of the lumbar porcine vertebrae being approximately $35 \mathrm{~mm}$ and $25 \mathrm{~mm}$, respectively [45]. These values are comparable with the human thoracic vertebrae (width T7-T10 29-35 mm, depth 28-32 mm) as described by Bozkus et al. [46]. Therefore, in absolute values, the size of the vertebrae are more comparable in the lumbar region of the porcine [42, 47]. The length of the spinous process is in the thoracic region of the porcine spine two to three times longer than the spinous process in the human spine [42]. In order to answer our specific research question, we considered the lumbar porcine spine suitable to predict the behaviour of the human spine. Most importantly, in the current study the effect of variation in rod stiffness on the intradiscal pressure of instrumented segments was assessed in a uniform, homogeneous spine model providing comparable biomechanical properties and geometry for each spine used.

The results of the current study suggest that dynamic fixation might lead to a decreased load on the bone screw interface, which may prevent instrumentation- and adjacent segment failure in the surgical treatment of degenerative spinal deformity. Further work to objectively compare pedicle screw pullout in rigid constructs and PCU constructs is currently ongoing in our laboratories.

\section{CONCLUSION}

In this concept study, the effect of spinal rod stiffness variation of a posterior stabilization device on the intradiscal pressure during cyclic flexion-compression loading conditions was assessed. Low implant stiffness was found to generate lower IDP, representing a more physiological loading pattern in comparison to high implant stiffness. Since a reduced spinal load has been associated with decreased stress at the screw-bone interface, the risk of instrumentation failure and PJF may potentially be reduced when using a stabilization device with lower implant stiffness. However, the optimal rod stiffness, achieving a predefined stabilization of the spine, remains unknown and requires further investigation. 


\section{REFERENCES}

1. Luo J, et al. Vertebral deformity arising from an accelerated "creep" mechanism. Eur Spine J, 2012. 21(9): p. 1684-91.

2. Ailon T, et al. Progressive Spinal Kyphosis in the Aging Population. Neurosurgery, 2015. 77 Suppl 4: p. S164-72.

3. Katzman WB, et al. Age-related hyperkyphosis: its causes, consequences, and management. J Orthop Sports Phys Ther, 2010. 40(6): p. 352-60.

4. Glassman SD, et al. The impact of positive sagittal balance in adult spinal deformity. Spine (Phila Pa 1976), 2005. 30(18): p. 2024-9.

5. Ailon T, et al. Degenerative Spinal Deformity. Neurosurgery, 2015. 77 Suppl 4: p. S75-91.

6. Goldstein CL, DS Brodke, and TJ Choma. Surgical Management of Spinal Conditions in the Elderly Osteoporotic Spine. Neurosurgery, 2015. 77 Suppl 4: p. S98-S107.

7. Macagno AE and MF O'Brien. Thoracic and thoracolumbar kyphosis in adults. Spine (Phila Pa 1976), 2006. 31(19 Suppl): p. S161-70.

8. Bastian L, et al. Evaluation of the mobility of adjacent segments after posterior thoracolumbar fixation: a biomechanical study. Eur Spine J, 2001. 10(4): p. 295-300.

9. Diebo BG, et al. Sagittal deformities of the spine: factors influencing the outcomes and complications. Eur Spine J, 2015. 24 Suppl 1: p. S3-15.

10. Annis $\mathrm{P}$, et al. Predictive factors for acute proximal junctional failure after adult deformity surgery with upper instrumented vertebrae in the thoracolumbar spine. Evid Based Spine Care J, 2014. 5(2): p. 160-2.

11. Halvorson TL, et al. Effects of bone mineral density on pedicle screw fixation. Spine (Phila Pa 1976), 1994. 19(21): p. 2415-20.

12. Okuyama K, et al. Stability of transpedicle screwing for the osteoporotic spine. An in vitro study of the mechanical stability. Spine (Phila Pa 1976), 1993. 18(15): p. 2240-5.

13. Ohtori $\mathrm{S}$, et al. Comparison of teriparatide and bisphosphonate treatment to reduce pedicle screw loosening after lumbar spinal fusion surgery in postmenopausal women with osteoporosis from a bone quality perspective. Spine (Phila Pa 1976), 2013. 38(8): p. E487-92.

14. Sun E, et al. Preventing distal pullout of posterior spine instrumentation in thoracic hyperkyphosis: a biomechanical analysis. J Spinal Disord Tech, 2009. 22(4): p. 270-7.

15. DeWald CJ and T Stanley. Instrumentation-related complications of multilevel fusions for adult spinal deformity patients over age 65: surgical considerations and treatment options in patients with poor bone quality. Spine (Phila Pa 1976), 2006. 31(19 Suppl): p. S144-51.

16. Ahn YH, et al. Comparison of the load-sharing characteristics between pedicle-based dynamic and rigid rod devices. Biomed Mater, 2008. 3(4): p. 044101.

17. Cabello J, et al. The protective role of dynamic stabilization on the adjacent disc to a rigid instrumented level. An in vitro biomechanical analysis. Arch Orthop Trauma Surg, 2013. 133(4): p. 443-8.

18. Gornet MF, et al. Biomechanical assessment of a PEEK rod system for semirigid fixation of lumbar fusion constructs. J Biomech Eng, 2011. 133(8): p. 081009.

19. Stoll TM, G Dubois and O Schwarzenbach. The dynamic neutralization system for the spine: a multi-center study of a novel non-fusion system. Eur Spine J, 2002. 11 Suppl 2: p. S170-8.

20. Schilling $\mathrm{C}$, et al. The effect of design parameters of dynamic pedicle screw systems on kinematics and load bearing: an in vitro study. Eur Spine J, 2011. 20(2): p. 297-307. 
21. Jahng TA, YE Kim and KY Moon. Comparison of the biomechanical effect of pedicle-based dynamic stabilization: a study using finite element analysis. Spine J, 2013. 13(1): p. 85-94.

22. Kurtz SM and JN Devine. PEEK biomaterials in trauma, orthopedic, and spinal implants. Biomaterials, 2007. 28(32): p. 4845-69.

23. Chou WK, A Chien, and JL Wang. Biomechanical analysis between PEEK and titanium screwrods spinal construct subjected to fatigue loading. J Spinal Disord Tech, 2015. 28(3): p. E121-5.

24. Schmidt H, F Heuer and HJ Wilke. Which axial and bending stiffnesses of posterior implants are required to design a flexible lumbar stabilization system? J Biomech, 2009. 42(1): p. 48-54.

25. Rohlmann A, et al. Comparison of the effects of bilateral posterior dynamic and rigid fixation devices on the loads in the lumbar spine: a finite element analysis. Eur Spine J, 2007. 16(8): p. 1223-31.

26. Khan I, et al. Analysis and evaluation of a biomedical polycarbonate urethane tested in an in vitro study and an ovine arthroplasty model. Part I: materials selection and evaluation. Biomaterials, 2005. 26(6): p. 621-31.

27. Wilke HJ, K Wenger and L Claes. Testing criteria for spinal implants: recommendations for the standardization of in vitro stability testing of spinal implants. Eur Spine J, 1998. 7(2): p. 148-54.

28. Hongo M, et al. Effect of multiple freeze-thaw cycles on intervertebral dynamic motion characteristics in the porcine lumbar spine. J Biomech, 2008. 41(4): p. 916-20.

29. Tan JS and S Uppuganti. Cumulative multiple freeze-thaw cycles and testing does not affect subsequent within-day variation in intervertebral flexibility of human cadaveric lumbosacral spine. Spine (Phila Pa 1976), 2012. 37(20): p. E1238-42.

30. Dath R, et al. Intradiscal pressure changes with dynamic pedicle screw systems. J Spinal Disord Tech, 2008. 21(4): p. 241-6.

31. Molz FJ, JI Partin and JS Kirkpatrick. The acute effects of posterior fusion instrumentation on kinematics and intradiscal pressure of the human lumbar spine. J Spinal Disord Tech, 2003. 16(2): p. 171-9.

32. Weinhoffer SL, et al. Intradiscal pressure measurements above an instrumented fusion. A cadaveric study. Spine (Phila Pa 1976), 1995. 20(5): p. 526-31.

33. Turner JL, DJ Paller and CB Murrell. The mechanical effect of commercially pure titanium and polyetheretherketone rods on spinal implants at the operative and adjacent levels. Spine (Phila Pa 1976), 2010. 35(21): p. E1076-82.

34. Schatzker J, JG Horne and G Sumner-Smith. The effect of movement on the holding power of screws in bone. Clin Orthop Relat Res, 1975(111): p. 257-62.

35. Galbusera F, et al. Pedicle screw loosening: a clinically relevant complication? Eur Spine J, 2015. 24(5): p. 1005-16.

36. Hwang JH, et al. Short segment pedicle screw fixation for unstable T11-L2 fractures: with or without fusion? A three-year follow-up study. Acta Orthop Belg, 2009. 75(6): p. 822-7.

37. Paxinos O, et al. Evaluation of pullout strength and failure mechanism of posterior instrumentation in normal and osteopenic thoracic vertebrae. J Neurosurg Spine, 2010. 13(4): p. 469-76.

38. Heuer F, et al. Stepwise reduction of functional spinal structures increase vertebral translation and intradiscal pressure. J Biomech, 2007. 40(4): p. 795-803.

39. Ferrara LA, et al. A biomechanical comparison of facet screw fixation and pedicle screw fixation - Effects of short-term and long-term repetitive cycling. Spine, 2003. 28(12): p. 1226-1234. 
40. Smit TH. The use of a quadruped as an in vivo model for the study of the spine - biomechanical considerations. Eur Spine J, 2002. 11(2): p. 137-44.

41. Busscher I, et al. In vitro biomechanical characteristics of the spine: a comparison between human and porcine spinal segments. Spine (Phila Pa 1976), 2010. 35(2): p. E35-42.

42. Busscher I. et al. Comparative anatomical dimensions of the complete human and porcine spine. Eur Spine J, 2010. 19(7): p. 1104-14.

43. Alini M, et al. Are animal models useful for studying human disc disorders/degeneration? Eur Spine J, 2008. 17(1): p. 2-19.

44. Cotterill PC, et al. An anatomical comparison of the human and bovine thoracolumbar spine. J Orthop Res, 1986. 4(3): p. 298-303.

45. Dath R, et al. Anatomical measurements of porcine lumbar vertebrae. Clin Biomech (Bristol, Avon), 2007. 22(5): p. 607-13.

46. Bozkus $\mathrm{H}$, et al. Comparative anatomy of the porcine and human thoracic spines with reference to thoracoscopic surgical techniques. Surg Endosc, 2005. 19(12): p. 1652-65.

47. Sheng SR, et al. Anatomy of large animal spines and its comparison to the human spine: a systematic review. Eur Spine J, 2010. 19(1): p. 46-56. 



\section{PART II}

\section{Degenerative Spinal Deformity}





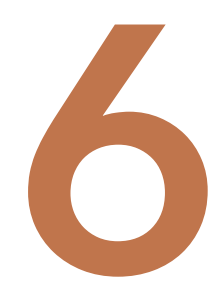

\section{The implementation of patient reported outcome} measures in appropriateness criteria of surgery for degenerative lumbar scoliosis

Jacobs E, van Kuijk SMJ, Merk JMR,

Vandewall-Peeters M, Jütten-Brouwer LMC, van Rhijn LW, Willems PC

Spine J. 2019 Apr;19(4):655-661 


\section{ABSTRACT}

Background context - Degenerative lumbar scoliosis (DLS) is an increasingly common spinal disorder of which current management is characterized by a substantial variety in treatment advice. To improve evidence-based clinical decision-making and increase uniformity and transparency of care, the Scoliosis Research Society established appropriateness criteria for surgery for DLS. In these criteria, however, the patient perspective was not formally incorporated. Since patient perspective is an increasingly important consideration in informed decision-making, embedding Patient-Reported Outcome Measures (PROMs) in the appropriateness criteria would allow for an objective and transparent patient-centered approach.

Purpose - To evaluate the extent that patient perspective is integrated into the appropriateness criteria of surgery for DLS.

Study design - Single center, retrospective, cohort study.

Patient sample - 150 patients with symptomatic degenerative lumbar scoliosis.

Outcome measures - The association between appropriateness for surgery and various PROMs [Visual Analogue Scale for pain, Short Form 36 (SF-36), Pain Catastrophizing Scale (PCS), Hospital Anxiety Depression Scale (HADS), and Oswestry Disability Index (ODI)].

Methods - Medical records of all patients with symptomatic DLS were reviewed and scored according to the appropriateness criteria. To assess the association between the appropriateness criteria and the validated PROMs, analysis of variance was used to test for differences in PROMS for each of the three categories resulting from the appropriateness criteria. To assess how well PROMs can discriminate between appropriate and inappropriate, we used a logistic regression analysis. Discriminative ability was subsequently determined by computing the area under the curve (AUC), resulting from the logistic regression analysis. Spearman rank analysis was used to establish a correlation pattern between the PROMs used and the appropriateness criteria.

Results - There was a significant association between the appropriateness of surgery and the PROMs. The discriminative ability for appropriateness of surgery for PROMs as a group was strong (AUC of 0.83). However, when considered in isolation, the predictive power of any individual PROMs was poor. The different categories of the appropriateness criteria significantly coincided with the PROMs used.

Conclusion - There is a statistically significant association between the appropriateness criteria of surgery for DLS and PROMs. Implementation of PROMs into the appropriateness criteria may lead to more transparent, quantifiable and uniform clinical decisionmaking for DLS. 


\section{INTRODUCTION}

The current management of de novo degenerative lumbar scoliosis (DLS) is characterized by a substantial variety of treatment options reflecting the absence of a clear, evidencebased, widespread stepwise approach [1]. Nonoperative treatment includes physical conditioning and exercise, pharmacological agents for pain control, use of orthotics, and pain interventions like epidural and facet injections [1-3]. Operative treatment can include instrumented stabilization and correction with posterior or anterior fusion, neurological decompression, or a combination of these $[1,3]$. Satisfactory clinical results of operative interventions have been reported [4-6]. However, the overall rate of complications for such major invasive surgery in predominantly elderly patients can exceed 30 percent [4].

To improve evidence-based clinical decision-making as well as increase uniformity, transparency, consistency and quality of care, the Scoliosis Research Society (SRS) assessed the appropriateness of surgery for DLS patients with specific clinical characteristics using the RAND/UCLA Appropriateness Method [1, 7-9]. Appropriateness criteria are quantitative tools designed to guide decision-making for well-defined populations of patients based on a combination of the best available evidence and multidisciplinary expert opinion [1, 7]. The aim of this method is to determine whether surgery for a particular patient is appropriate or not. When expected harms outweigh expected benefits, the procedure would be considered inappropriate [1].

Patient Reported Outcome Measures (PROMs) are now widely used in clinical practice to narrow the gap between the clinician's and patient's view of what constitutes a clinically meaningful improvement and to help tailor treatment plans to meet the patient's preferences and needs. PROMs quantify patients' perspectives on the severity of their symptoms, the impact of the disease on their functioning, and the degree to which it limits their health-related quality of life [10]. In the SRS appropriateness criteria for DLS the patient perspective has not been formally incorporated [1]. The degree of symptoms in the appropriateness criteria is currently based on the patient's reported symptoms as they are perceived during outpatient assessment by the treating surgeon and is thereby susceptible for interpretation. Because patient perspective is an increasingly important consideration in informed decision-making, embedding PROMs into the appropriateness criteria for DLS would allow for a more quantifiable and transparent patient-centered approach in clinical decision-making.

The purpose of this study was to evaluate whether there is an association between the appropriateness criteria for degenerative lumbar scoliosis and the patient perspective as determined by validated health-related quality of life (HRQOL) instruments or PROMs. 


\section{MATERIALS AND METHODS}

For this retrospective study, the medical records of all consecutive DLS patients who had been treated at the multidisciplinary spine center of the Maastricht University Medical Centre (the Netherlands) between January 1, 2011 through December 31, 2017 were analyzed. Institutional review board (IRB) approval was obtained before study initiation (METC17-4-022). We included adult patients with de novo DLS with a Cobb angle of more than 10 degrees in the coronal plane, available Patient Reported Outcome Measures (PROMs), and available standing two direction full spine or lumbar spine plain radiographs $[2,3]$. Patients were excluded if they were suffering from idiopathic adolescent scoliosis or neuromuscular or congenital scoliosis, had undergone previous corrective spinal surgery and fusion, or complete data was unavailable.

For each patient, medical records and radiographs were reviewed and scored according to the SRS appropriateness criteria [1], which is comprised of seven clinical or radiographic characteristics: (1) severity of self-reported symptoms, (2) severity of central spinal or foraminal stenosis, (3) progression of the degree of curvature or certain other radiographic abnormalities, (4) presence of sagittal imbalance, (5) severity of risk factors for suboptimal outcomes, (6) degree of curvature, and (7) when applicable, number of levels with at least moderate central spinal or foraminal stenosis. For the category "progression", plain radiographs at various time points were assessed (at least 3 months apart). However, in a few cases, radiographs were only available for one time point in which case "no progression" was scored (in accordance with the appropriateness criteria [1]). Based on the appropriateness criteria for each patient it was determined whether or not surgery would have been inappropriate, appropriate or necessary. To assess agreement between raters (inter-observer reliability), a random sample of 26 patients was reviewed and scored by two different raters (E.J. a resident orthopedic surgery and P.W. an orthopedic surgeon with 15 years of experience in spine surgery).

Clinical patient analysis was based on standard PROMs which were collected in standard care at the same day of radiographic acquisition and included the following instruments: visual analogue scale (VAS) for back and leg pain, Short Form 36 (SF-36), Pain Catastrophizing Scale (PCS), Hospital Anxiety Depression Scale (HADS), and, for the majority of patients, the Oswestry Disability Index (ODI). While higher scores on the VAS, PCS, HADS and ODI represent increasing pain and disability, the inverse holds true for the SF-36 instrument, in which higher scores reflect less disability or pain.

\subsection{Statistical Analysis}

We had no prior hypotheses on the strength of the associations between PROMs and the appropriateness criteria. Therefore, no formal sample size calculation was performed based on the preferred power to detect such an association. We included all evaluable patients treated within the selected period of time and judged appropriateness of the 
size of our cohort using general recommendations for ANOVA and (logistic) regression (i.e., 10 observations per variable for continuous outcomes, 10 events per variable for dichotomous outcomes). With these in mind, 150 subjects would provide ample precision to reliably estimate the suggested associations.

Baseline characteristics of the patients were described using mean and standard deviation (SD) for continuous variables and as count and percentage for categorical variables. Subsequently, ordinal logistic regression analysis was used to determine which of the variables of the appropriateness criteria (i.e. degree of symptoms, degrees of the curve) was most associated with the outcome (inappropriate, appropriate or necessary).

To assess inter-observer agreement on the scoring of medical records and radiographs, we computed overall agreement and Cohen's Kappa coefficient to correct for chance agreement.

To assess the association between the outcome of the appropriateness criteria and the patient perspective, analysis of variance (ANOVA) was used to test for differences in PROMs for each of the three categories resulting from the appropriateness criteria (i.e. inappropriate, appropriate, necessary). In case of severe non-normality, we used the Kruskal-Wallis test. Subsequently, the appropriateness criteria were dichotomized into two categories: appropriate (including both appropriate and necessary) and inappropriate. To assess how well PROMs can discriminate between appropriate and inappropriate, we used logistic regression analysis. Discriminative ability was subsequently determined by computing the area under the Receiver Operating Characteristic (ROC) curve, or AUC, resulting from the logistic regression analysis.

Correlations between the various PROMs and the separate variables contributing to the appropriateness criteria were estimated using the Spearman rank order correlation coefficient. All analyses were performed using R version 3.5.1.

\section{RESULTS}

Medical records of 195 patients were reviewed and a total of 150 lumbar degenerative deformity patients met the inclusion criteria and were included for analysis. The study group consisted of 28 men and 122 women, and the average age of the study sample was 67.1 years $(\mathrm{SD}=9.37$, range 42 to 89 years). The mean Cobb angle was 29.6 degrees ( $\mathrm{SD}=$ 11.3, range 11 to 64 degrees). The distribution of patients according to the appropriateness criteria is shown in Table 1.

According to the appropriateness criteria, surgery was inappropriate for 60 patients (mean Cobb angle 22.4+8.7 degrees), appropriate for 49 patients (mean Cobb angle 32+10 degrees) and necessary for 41 patients (mean Cobb angle 37.3+10 degrees). Ordinal logistic regression results showed that the appropriateness of surgery is most significantly dependent on the degree of symptoms, imbalance and magnitude of curvature (Table 2). 
Table 1. The distribution of patients according to the appropriateness criteria as defined by the Scoliosis Research Society (data shown as number of patients [percentage of total]) [4]. See http:/ links.lww.com/BRS/B79 for definitions.

\begin{tabular}{|c|c|c|c|c|}
\hline & Inappropriate & Appropriate & Necessary & Total \\
\hline \multicolumn{5}{|c|}{ Degree of symptoms } \\
\hline None & $0(0)$ & $2(1)$ & $0(0)$ & $2(1)$ \\
\hline Mild & $55(37)$ & $14(9)$ & $0(0)$ & $69(46)$ \\
\hline Moderate & $5(3)$ & $33(22)$ & $35(23)$ & $73(49)$ \\
\hline Severe & $0(0)$ & $0(0)$ & $6(4)$ & $6(4)$ \\
\hline \multicolumn{5}{|c|}{ Degree of stenosis } \\
\hline None & $32(21)$ & $17(11)$ & $6(4)$ & $55(37)$ \\
\hline Mild & $26(17)$ & $15(10)$ & $14(9)$ & $55(37)$ \\
\hline Moderate & $2(1)$ & $17(11)$ & $20(13)$ & $39(26)$ \\
\hline Severe & $0(0)$ & $0(0)$ & $1(1)$ & $1(\mathbf{1})$ \\
\hline \multicolumn{5}{|l|}{ Progression } \\
\hline No & $60(40)$ & $48(32)$ & $41(27)$ & 149 (99) \\
\hline Yes & $0(0)$ & $1(1)$ & $0(0)$ & $1(1)$ \\
\hline \multicolumn{5}{|l|}{ Imbalance } \\
\hline No & $58(39)$ & $24(16)$ & $2(1)$ & $84(56)$ \\
\hline Yes & $2(1)$ & $25(17)$ & $39(26)$ & $66(44)$ \\
\hline \multicolumn{5}{|l|}{ Risk factors } \\
\hline None to mild & $28(19)$ & $20(13)$ & $16(11)$ & $64(43)$ \\
\hline Moderate & $28(19)$ & $26(17)$ & $23(15)$ & $77(51)$ \\
\hline Severe & $4(3)$ & $3(2)$ & $2(1)$ & $9(6)$ \\
\hline \multicolumn{5}{|l|}{ Curvature } \\
\hline Mild $\left(10-19^{\circ}\right)$ & $27(18)$ & $5(3)$ & $0(0)$ & $32(21)$ \\
\hline Moderate $\left(20-29^{\circ}\right)$ & $24(16)$ & $10(7)$ & $8(5)$ & $42(28)$ \\
\hline Moderate $\left(30-39^{\circ}\right)$ & $7(5)$ & 25 (17) & $17(11)$ & $49(33)$ \\
\hline Severe $\left(\geq 40^{\circ}\right)$ & $2(1)$ & $9(6)$ & $16(11)$ & 27 (18) \\
\hline TOTAL & $60(40)$ & $49(33)$ & $41(27)$ & 150 (100) \\
\hline
\end{tabular}

Table 2. Outcome of the ordinal logistic regression analysis, regressing the appropriateness of surgery against the various variables of the appropriateness criteria.

\begin{tabular}{lcccc}
\hline & Coefficient & Standard error & T-value & P-value \\
\hline Degree of symptoms & 8.00 & 1.85 & 4.33 & $0.00^{*}$ \\
Degree of stenosis & 2.65 & 0.83 & 3.18 & $0.00^{*}$ \\
Progression & 6.36 & 13.79 & 0.46 & 0.65 \\
Imbalance & 9.66 & 2.04 & 4.74 & $0.00^{*}$ \\
Risk factors & -3.37 & 1.12 & -3.00 & $0.00^{*}$ \\
Curvature & 2.34 & 0.68 & 3.44 & $0.00^{*}$ \\
Constant & & & & 0.01 \\
Inappropriate vs appropriate & 43.31 & 16.48 & 2.63 & \\
Appropriate vs necessary & 55.17 & 18.02 & 3.06 & 0.00 \\
\hline
\end{tabular}

Ordinal logistic regression analysis demonstrated that appropriateness for surgery is most significantly dependent on the degree of symptoms, imbalance and curvature. 
The absolute inter-observer agreement between the two raters of the medical records and radiographs to determine the appropriateness category was 92.3\%. Adjusted for chance-agreement, the Kappa coefficient was 0.88 on a scale from 0 to 1 ( $p<0.001$ ). The high Kappa score indicated that there was high consensus in the ratings given by the two raters.

Table 3 shows results of the ANOVA and in case of skewed variables Kruskal-Wallis statistics. It revealed a significant association between the appropriateness of surgery and virtually all PROMs (VAS, PCS, HADS, ODI). Only three domains of the SF-36 (energy, role limitations physical and emotional) showed no significant association with the appropriateness of surgery.

Table 3. Association between the outcome of the appropriateness criteria and the PROMs (data shown as mean / median [range]).

\begin{tabular}{|c|c|c|c|c|}
\hline & Inappropriate & Appropriate & Necessary & $\begin{array}{c}\text { P-value } \\
\text { for difference }\end{array}$ \\
\hline \multicolumn{5}{|l|}{ VAS } \\
\hline Right leg & 27 / 10 [0-100] & 35 / 20 [0-100] & 45 / $50[0-100]$ & 0.01 \\
\hline Left leg & $20 / 0[0-100]$ & $31 / 20[0-100]$ & 45 / $50[0-100]$ & 0.00 \\
\hline Spine & 58 / 60 [0-100] & 61 / 65 [0-100] & 74 / 75 [30-100] & 0.00 \\
\hline \multicolumn{5}{|l|}{ SF-36 } \\
\hline Physical functioning* & 44 / 45 [0-90] & 36 / 30 [0-100] & $24 / 20$ [0-75] & 0.00 \\
\hline Social functioning* & $60 / 63[0-100]$ & 60 / 63 [13-100] & $42 / 50[0-88]$ & 0.00 \\
\hline Role limitations (physical)* & $27 / 0[0-100]$ & $23 / 0$ [0-100] & 15 / 0 [0-100] & 0.17 \\
\hline Role limitations (emotional)* & 62 / 67 [0-100] & $50 / 33$ [0-100] & 45 / 33 [0-100] & 0.09 \\
\hline Emotional well-being* & $71 / 76$ [4-92] & 65 / 68 [12-96] & 62 / 64 [16-100] & 0.03 \\
\hline Energy (fatigue) & 49 / 50 [0-85] & 47 / 50 [10-85] & 43 / 45 [10-85] & 0.08 \\
\hline Pain & $41 / 45[0-80]$ & 36 / 45 [0-88] & $28 / 22[0-100]$ & 0.00 \\
\hline General health & 51 / 53 [10-90] & $50 / 50[5-80]$ & 42 / 40 [0-75] & 0.01 \\
\hline Health change* & 22 / 25 [0-75] & $31 / 25$ [0-50] & $21 / 25$ [0-50] & 0.01 \\
\hline PCS & 19 / 17 [0-52] & 25 / 25 [1-52] & 27 / 26 [3-48] & 0.00 \\
\hline \multicolumn{5}{|l|}{ HADS } \\
\hline Anxiety* & 5 / 5 [0-19] & 7 / 6 [1-16] & 8 / 8 [0-17] & 0.02 \\
\hline Depression* & 5 / 4 [0-17] & 6 / 6 [0-18] & 8 / 7 [1-17] & 0.01 \\
\hline ODI & 33 / 32 [6-66] & $41 / 44$ [0-70] & 51 / 51 [30-72] & 0.00 \\
\hline
\end{tabular}

VAS indicates Visual Analogue Scale; SF-36: Short Form 36, PCS: Pain Catastrophizing Scale; HADS: Hospital Anxiety Depression Scale; ODI: Oswestry Disability Index. *Because of skewness of the data the Kruskal-Wallis test was used. 
The ability of PROMs to discriminate between those for whom surgery would be deemed inappropriate according to the criteria and those for whom surgery would be deemed appropriate or necessary was expressed as the AUC. The AUC for the combined VAS scores (i.e. VAS right leg, left leg, and spine), was 0.69 (95\% confidence interval [CI]: 0.60 - 0.77), for the SF-36 domains 0.70 (95\% CI: 0.61 - 0.79), for PCS 0.67 (95\% CI: $0.57-0.76$ ), for the combined HADS domains 0.65 (95\% CI: $0.56-0.74$ ), and for the ODI 0.73 (95\% CI: $0.61-0.85$ ). For a model combining all PROMs, the AUC was 0.83 (95\% CI: $0.71-0.95$ ).

Table 4. Spearman rank order correlation coefficient analysis for the various components of the appropriateness criteria and the different PROMs (analysis based on aggregate data).

\begin{tabular}{|c|c|c|c|c|c|c|c|}
\hline & $\begin{array}{l}\text { VAS } \\
\text { spine }\end{array}$ & $\begin{array}{c}\text { VAS } \\
\text { left leg }\end{array}$ & $\begin{array}{l}\text { VAS } \\
\text { right leg }\end{array}$ & PCS & $\begin{array}{c}\text { HADS } \\
\text { anxiety }\end{array}$ & $\begin{array}{c}\text { HADS } \\
\text { depression }\end{array}$ & ODI \\
\hline Symptoms & $0.43^{*}$ & $0.21^{*}$ & $0.22^{*}$ & $0.34^{*}$ & $0.27^{*}$ & $0.32^{*}$ & $0.48^{*}$ \\
\hline Degree of stenosis & 0.11 & $0.56^{*}$ & $0.60^{*}$ & $0.24^{*}$ & 0.15 & $0.19^{* *}$ & $0.29^{*}$ \\
\hline Progression & -0.09 & 0.00 & 0.02 & -0.07 & -0.11 & -0.08 & NA \\
\hline Imbalance & 0.13 & 0.07 & -0.04 & $0.21^{* *}$ & 0.14 & $0.18^{* *}$ & $0.35^{*}$ \\
\hline Risk factors & $0.24^{*}$ & 0.03 & 0.03 & $0.20^{* *}$ & $0.18^{* *}$ & $0.27^{*}$ & $0.28^{* *}$ \\
\hline Curvature & 0.07 & 0.11 & 0.02 & 0.13 & 0.10 & 0.10 & 0.18 \\
\hline Levels & $0.18^{* *}$ & $0.28^{*}$ & $0.36^{*}$ & $0.23^{* *}$ & 0.13 & $0.17^{* *}$ & 0.18 \\
\hline
\end{tabular}

${ }^{*}$ indicate $\mathrm{p}<0.01,{ }^{* *}$ indicate $\mathrm{p}<0.05$.

Table 5. Spearman rank order correlation coefficient analysis for the various components of the appropriateness criteria and the Short-From 36 (analysis based on aggregate data).

\begin{tabular}{|c|c|c|c|c|c|c|c|c|c|}
\hline & 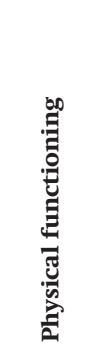 & 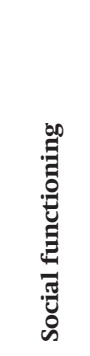 & 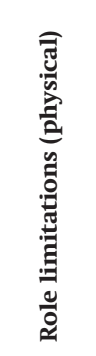 & 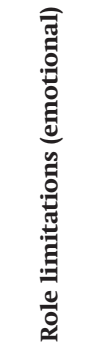 & 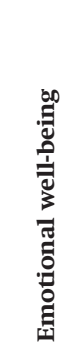 & 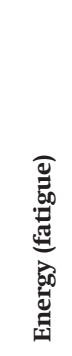 & 灵 & 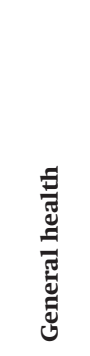 & 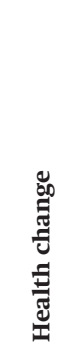 \\
\hline Degree of symptoms & $-0.47^{*}$ & $-0.36^{*}$ & $-0.23^{*}$ & $-0.22^{*}$ & $-0.23^{*}$ & $-0.3^{*}$ & $-0.39 *$ & $-0.28^{*}$ & -0.20 \\
\hline Degree of stenosis & $-0.21^{*}$ & $-0.24^{*}$ & -0.20 & -0.16 & $-0.23^{*}$ & -0.14 & $-0.37^{*}$ & -0.19 & -0.12 \\
\hline Progression & 0.06 & 0.13 & 0.06 & -0.10 & 0.06 & 0.11 & 0.11 & 0.11 & -0.02 \\
\hline Imbalance & $-0.22^{*}$ & -0.12 & -0.05 & -0.12 & -0.14 & -0.08 & -0.08 & -0.15 & -0.09 \\
\hline Risk factors & $-0.31^{*}$ & -0.17 & -0.1 & $-0.21^{*}$ & -0.2 & -0.13 & -0.11 & -0.18 & -0.05 \\
\hline Curvature & 0.00 & -0.02 & -0.04 & -0.08 & -0.10 & 0.02 & -0.02 & -0.05 & -0.09 \\
\hline Levels & $-0.25 *$ & -0.2 & -0.17 & -0.10 & -0.17 & -0.17 & $-0.26^{*}$ & -0.14 & -0.04 \\
\hline
\end{tabular}

${ }^{*}$ indicate $\mathrm{p}<0.01$. 
Table 6. Association between the various subcategories as defined by the appropriateness criteria and their "matching” PROMs (data shown as mean / median [range]).

\begin{tabular}{|c|c|c|c|c|c|}
\hline Degree of symptoms & None & Mild & Moderate & Severe & P-value \\
\hline VAS spine & $20 / 20[0-40]$ & 53 / $50[0-100]$ & 73 / 75 [0-100] & 79 / 78 [70-100] & 0.00 \\
\hline SF-36 phys. functioning & 65 / 65 [50-80] & 48 / $45[0-100]$ & 25 / 20 [0-75] & $23 / 20[5-50]$ & 0.00 \\
\hline ODI & $\mathrm{NA}^{*}$ & 31 / 29 [0-66] & 48 / 50 [18-70] & 54 / 60 [32-72] & 0.00 \\
\hline Degree of stenosis & None & Mild & Moderate & Severe & \\
\hline VAS right leg & $7 / 0[0-70]$ & 49 / $50[0-100]$ & 54 / 60 [0-100] & $\mathrm{NA}^{*}$ & 0.05 \\
\hline VAS left leg & $6 / 0[0-90]$ & 39 / 40 [0-100] & 53 / 60 [0-100] & $\mathrm{NA}^{*}$ & 0.08 \\
\hline Imbalance & No & Yes & & & \\
\hline ODI & 36 / 34 [6-70] & 47 / 50 [0-72] & & & 0.00 \\
\hline Risk factors & None to mild & Moderate & Severe & & \\
\hline SF-36 role limit. (emotional) & $61 / 67[0-100]$ & 52 / 67 [0-100] & 7 / 0 [0-33] & & 0.00 \\
\hline SF-36 emotional well-being & $71 / 76$ [24-100] & 65 / 68 [4-96] & 56 / 60 [28-72] & & 0.04 \\
\hline PCS & 21 / 21 [2-51] & 24 / 24 [0-52] & 34 / 33 [15-44] & & 0.03 \\
\hline HADS anxiety & 6 / 5 [0-17] & 7 / 6 [0-19] & 9 / 8 [5-12] & & 0.04 \\
\hline HADS depression & 6 / 5 [0-17] & 7 / 6 [0-18] & 10 / 8 [6-16] & & 0.00 \\
\hline
\end{tabular}

* Indicates too little observations.

As demonstrated in Table 4 to 6 , the Spearman rank order correlation coefficients showed a statistically significant correlation between the degree of symptoms and each PROM ( $\mathrm{p}<0.01$ ). Another statistically significant positive correlation was found between the VAS leg scores (left and right) and the degree of stenosis ( $\rho$ 0.56 (VAS left leg), $\rho 0.60$ (VAS right leg), $\mathrm{p}<0.01$ ). Moreover, the Spearman rank order correlation coefficients also demonstrated a statistically significant correlation between the appropriateness criteria and adverse health status score for all domains of the SF-36 score (p < 0.01) (Table 5) and a statistically significant positive correlation with the PCS score $(\mathrm{p}<0.01)$, the HADS scores $(\mathrm{p}<0.01)$ and the ODI questionnaires $(\mathrm{p}<0.01)$ (Table 4).

\section{DISCUSSION}

In the current study, a statistically significant association between the appropriateness of surgery for DLS and validated Patient Reported Outcome Measures (PROMs) was found. Patients for whom surgery was deemed necessary showed a higher degree of pain and disability, whereas the inverse holds true for patients for whom surgery would have been inappropriate. The appropriateness criteria for DLS as developed by the Scoliosis Research Society represent a significant step towards evidence based uniform treatment in spinal surgery [1]. However, as stated by Glassman et al. [7], it is important to recognize the appropriateness criteria of surgery for DLS are a starting point, not an end point. 
From this statement one can infer that the current appropriateness criteria for DLS require further validation and refinement. One important limitation of the current criteria for DLS is the absence of Patient Reported Outcome Measures (PROMs) as a validated, uniform and transparent measure of patient perspective [1, 7].

In this study imbalance appeared to be the strongest determinant for appropriateness of surgery (Table 2, T-value 4.74). Imbalance was associated with a higher degree of pain and disability in patients suffering from DLS (Tables 4 and 5), which is in accordance with recent literature [11-14]. In a recent study Daubs et al. [15], also using the RAND/ UCLA Appropriateness Method, demonstrated that sagittal imbalance was a major factor affecting the appropriateness of surgery among patients with DLS.

A patient's health condition is often mainly determined by the subjective observation of the treating spinal surgeon and, as such, is highly susceptible to interpretation. PROMs, on the other hand, are defined as "Any report of the patient's health condition that comes directly from the patient, without interpretation of the patient's response by a clinician or anyone else" [16]. By using individual patient PROMs, clinicians receive standardized information on a patient's individual health problem in order to identify or monitor symptoms and support shared decision-making. As shown in the current study, the discriminative ability for appropriateness of surgery for the PROMs as a group is strong (AUC 0.83 (95\% CI:0.71 - 0.95)), however, when considered in isolation the predictive power of the individual PROMs is poor (AUC between 0.65 and 073). For example, it feels contradictory that in the current study a high PCS and HADS score were positively associated with the appropriateness of surgery. In recent literature, it has been shown that preoperative depression is significantly associated with decreased improvement in quality of life after lumbar surgery [17]. Although there is a statistically significant association between the PCS or HADS and the category "risk factors" of the appropriateness criteria, this category apparently has little impact on the appropriateness of surgery. These findings support the use of preoperative PROMs (including a validated psychosocial questionnaire) as an additional tool for determination of appropriateness of surgery and for optimization of surgical outcome. In an effort to maximize the benefit of surgery, a trained multidisciplinary team may then address and treat anxiety or depression before proceeding with a surgical intervention.

Although, the current study advocates the implementation of quantifiable, transparent PROMs into the appropriateness criteria, it should be further elucidated which PROMs can be used best and how they should be interpreted. Tables 4 and 5 show by means of a correlation analysis that the different categories of the appropriateness criteria significantly correlate with the various PROMs. The various PROMS all represent different components of the patient's health-related quality of life, and subsequently correspond with the different subcategories of the appropriateness criteria. As shown in Table 6, the VAS spine quantifies the amount of perceived back pain, the VAS leg defines the amount of perceived leg pain, the ODI questionnaire quantifies how DLS impacts functioning and 
health-related quality of life, and the HADS score demonstrates the level of depression and anxiety a patient experiences (subcategory risk factors). As an example, each PROM could be added to reinforce the corresponding appropriateness category. However, the exact thresholds of these individual PROMS to advise for or against surgery are yet to be determined. To determine such thresholds, and to determine which exact PROMs should be used, prospective studies with larger patient cohorts or analyses from spine registries will be necessary. Furthermore, patient preference with respect to surgery is not addressed by the PROMs or the appropriateness criteria itself. More insight into the patient preference could provide valuable information for clinical decision-making in patients suffering from DLS. Finally, as PROMs reflect the individually perceived burden caused by a spinal disorder, their implementation into appropriateness criteria should account for cultural differences as well.

In conclusion, this study has revealed statistically significant association between validated PROMs and the appropriateness criteria for surgery in DLS. Implementation of PROMs into the appropriateness criteria could provide a more quantifiable, transparent and uniform approach. Future studies with larger patient cohorts should further validate the incorporation of PROMs into the appropriateness criteria and optimize thresholds when to opt for surgery or not in DLS. 


\section{REFERENCES}

1. Chen PG, Daubs MD, Berven S, et al. Surgery for Degenerative Lumbar Scoliosis: The Development of Appropriateness Criteria. Spine (Phila Pa 1976) 2016;41(10):910-8 doi: 10.1097/ BRS.0000000000001392.

2. Benner B, Ehni G. Degenerative lumbar scoliosis. Spine (Phila Pa 1976) 1979;4(6):548-52

3. Aebi M. The adult scoliosis. Eur Spine J 2005;14(10):925-48 doi: 10.1007/s00586-005-1053-9.

4. Yamada K, Nakamae T, Shimbo T, et al. Targeted Therapy for Low Back Pain in Elderly Degenerative Lumbar Scoliosis: A Cohort Study. Spine (Phila Pa 1976) 2016;41(10):872-9 doi: 10.1097| BRS.0000000000001524.

5. Takahashi S, Delecrin J, Passuti N. Surgical treatment of idiopathic scoliosis in adults: an age-related analysis of outcome. Spine (Phila Pa 1976) 2002;27(16):1742-8

6. Shapiro GS, Taira G, Boachie-Adjei O. Results of surgical treatment of adult idiopathic scoliosis with low back pain and spinal stenosis: a study of long-term clinical radiographic outcomes. Spine (Phila Pa 1976) 2003;28(4):358-63 doi: 10.1097/01.BRS.0000048502.62793.0C.

7. Glassman SD, Berven SH, Shaffrey CI, Mummaneni PV, Polly DW. Commentary: Appropriate Use Criteria for Lumbar Degenerative Scoliosis: Developing Evidence-based Guidance for Complex Treatment Decisions. Neurosurgery 2017;80(3):E205-E12 doi: 10.1093/neuros/ nyw094.

8. Lawson EH, Gibbons MM, Ingraham AM, Shekelle PG, Ko CY. Appropriateness criteria to assess variations in surgical procedure use in the United States. Arch Surg 2011;146(12):1433-40 doi: 10.1001/archsurg.2011.581.

9. Shekelle P. The appropriateness method. Med Decis Making 2004;24(2):228-31 doi: 10.1177/0272989X04264212[published Online First: Epub Date]|.

10. Falavigna A, Dozza DC, Teles AR, et al. Current Status of Worldwide Use of Patient-Reported Outcome Measures (PROMs) in Spine Care. World Neurosurg 2017;108:328-35 doi: 10.1016/j. wneu.2017.09.002.

11. Glassman SD, Bridwell K, Dimar JR, Horton W, Berven S, Schwab F. The impact of positive sagittal balance in adult spinal deformity. Spine (Phila Pa 1976) 2005;30(18):2024-9

12. Glassman SD, Berven S, Bridwell K, Horton W, Dimar JR. Correlation of radiographic parameters and clinical symptoms in adult scoliosis. Spine (Phila Pa 1976) 2005;30(6):682-8

13. Mac-Thiong JM, Transfeldt EE, Mehbod AA, et al. Can c7 plumbline and gravity line predict health related quality of life in adult scoliosis? Spine (Phila Pa 1976) 2009;34(15):E519-27 doi: 10.1097/BRS.0b013e3181a9c7ad.

14. Schwab FJ, Blondel B, Bess S, et al. Radiographical spinopelvic parameters and disability in the setting of adult spinal deformity: a prospective multicenter analysis. Spine (Phila Pa 1976) 2013;38(13):E803-12 doi: 10.1097/BRS.0b013e318292b7b9.

15. Daubs MD, Brara HS, Raaen LB, et al. How does sagittal imbalance affect the appropriateness of surgical indications and selection of procedure in the treatment of degenerative scoliosis? findings from the RAND auc study. Spine J 2018 doi: 10.1016/j.spinee.2018.01.027.

16. Santana MJ, Haverman L, Absolom K, et al. Training clinicians in how to use patient-reported outcome measures in routine clinical practice. Qual Life Res 2015;24(7):1707-18 doi: 10.1007/ s11136-014-0903-5.

17. Miller JA, Derakhshan A, Lubelski D, et al. The impact of preoperative depression on quality of life outcomes after lumbar surgery. Spine J 2015;15(1):58-64 doi:10.1016/j.spinee.2014.06.020. 




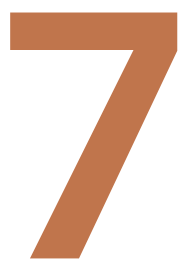

\section{Prediction of mechanical} complications in adult spinal deformity surgery The GAP score versus the Schwab classification

Jacobs E, van Royen BJ, van Kuijk SMJ, Merk, JMR, Stadhouder A, van Rhijn LW, Willems PC

Spine J. 2019 May;19(5):781-788 


\section{ABSTRACT}

Background context - Surgery for adult spinal deformity (ASD) is a challenging and complex procedure with high reported complication (8.4-42\%) and revision rates (917.6\%). Failure to achieve or maintain adequate postoperative sagittal alignment has been reported to be the main cause of mechanical complications. In order to define appropriate surgical targets, the Scoliosis Research Society (SRS)-Schwab classification and the Global Alignment and Proportion (GAP) score were established. In the literature, no study has yet compared these classification systems with respect to the risk of developing mechanical complications.

Purpose - To assess and compare the ability of the Schwab classification and the GAP score to predict mechanical complications following adult spinal deformity surgery.

Study design - Two center, retrospective cohort study.

Patient sample - Thirty-nine patients suffering ASD who underwent long segment spinal fusion ( $\geq 4$ levels), minimum follow-up of 2 years.

Outcome measures - The ability of the Schwab classification and GAP score to predict mechanical failure was determined by computing the Area Under the Receiver Operating Characteristic Curve (AUC).

Methods - Full-spine pre- and postoperative radiographs of all patients were analyzed for mechanical complications. Subsequently the pre- and postoperative Schwab and GAP score was determined. Logistic regression analysis was used to assess the ability of both systems to determine which was the most appropriate for the prediction of mechanical failure. Correlations between the various factors constituting the GAP score and Schwab classification were estimated using the Spearman rank order correlation coefficient.

Results - The results demonstrated that both classification systems are capable of predicting radiographic evidence of mechanical failure, however the GAP score proved to be significantly better $(\mathrm{p}=0.003$ ). The relative pelvic version (RPV) of the GAP score serves a similar role as the pelvic tilt (PT) modifier from the Schwab classification $(\rho=-0.84$, $\mathrm{p}<0.01$ ). The relative lumbar lordosis (RLL) from the GAP score functions much like the PI-LL modifier from the Schwab classification $(\rho=-0.94, \mathrm{p}<0.01)$. The GAP score is most significantly dependent on RSA, RLL and RPV $(\rho=0.85, \rho=0.84$, and $\rho=0.84$, respectively, $\mathrm{p}<0.01$ ). Correlation with the lordosis distribution index (LDI) was also significant but was not as strong $(\rho=0.65, p<0.01)$. Age, on the contrary, showed poor correlation with the GAP score $(\rho=0.17, p=0.300)$.

Conclusion - Both the Schwab classification and the GAP score are capable of predicting mechanical complications. The GAP score proved to be significantly more appropriate. 
This difference is probably attributed to the fact that in the GAP score all parameters are related to the patient's individual pelvic incidence.

\section{INTRODUCTION}

Degenerative changes have the potential to greatly disrupt the normal curvature of the spine, leading to sagittal malalignment [1]. The subsequent pain and decline in functional status constitute a concerning clinical picture [2]. As a response to positive sagittal (mal) alignment, the body progressively recruits compensatory mechanisms to counteract the anterior truncal shift [1]. A chain of compensation is initiated from the flexible parts of the spine, and extends to the hips, lower extremities, and cervical spine to preserve an erect posture and horizontal gaze. The interaction between deformity and compensatory mechanisms depicts the final presentation of patients with adult spinal deformity (ASD) [3]. In cases of severe deformity, surgical intervention has been shown to offer superior clinical and radiographic outcomes compared to non-operative approaches [4-6].

Understanding of the sagittal alignment of the spine allows for comprehensive surgical planning which is key to achieving optimum postoperative alignment and improved outcomes [3]. Recent studies on outcomes following ASD surgeries have shown high rates of complications $(8.4-42 \%)[7,8]$ and revision rates $(9-17.6 \%)[3,9]$. Increased junctional stress concentration causes soft-tissue and ligamentous failure, vertebral fracture and bone implant interface failure. Proximal junctional kyphosis (PJK) and proximal junctional failure (PJF) are recognized complications of ASD surgery with a reported prevalence of $20-40 \%[3,10,11]$. The development of junctional failure is frequently symptomatic and can lead to the need for revision surgery and can have potential catastrophic neurological sequelae [12]. The major risk factors for junctional failure include older age (over 55 years of age), large abnormal preoperative sagittal parameters, osteoporosis, high body mass index, thoracoplasty procedures and fusion to the lower lumbar vertebra and sacrum [11, 13-15].

Adequate alignment following surgical treatment for ASD is not consistently achieved. Since revision rates following realignment procedures increase progressively with longer follow-up, it is possible that these revisions are driven by failure to achieve and/or maintain realignment $[1,3]$. Both under- and over-correction in the sagittal plane have been reported to be a main cause of mechanical complications [12, 16-18]. Nowadays, extensive research has converted theoretical concepts into clinically relevant guidelines on sagittal alignment. In treatment planning for ASD accurate sagittal alignment analysis has become an essential tool. In order to understand appropriate targets for the correction of ASD, the Scoliosis Research Society (SRS)-Schwab classification and the Global Alignment and Proportion (GAP) score have been developed [19, 20]. The SRS-Schwab classification uses three sagittal modifiers to quantify deformity [19]. The Schwab modifier 
thresholds were based on the established correlations between radiographic parameters and health-related quality-of-life measures [19, 21]. However, despite achieving optimal Schwab values postoperatively, mechanical complications are not uncommon [22]. Yilgor et al. [20] noticed that it was not clear how the Schwab criteria influence the risk of mechanical complications. Therefore, they developed the GAP score in which thresholds were mainly determined on the risk of mechanical complications. In the GAP score, optimal sagittal alignment is based on four factors deviating from their ideal curves, and these factors are proportionally related to the pelvic incidence (PI) [20]. The overall goal of the GAP score is to achieve a more patient-tailored, or patients-specific, guide for spinopelvic alignment. To date, no study has yet compared the SRS-Schwab classification with the newly developed GAP score with respect to their ability to predict the risk of developing mechanical complications following ASD surgery. Therefore, the purpose of the current study is to assess whether the SRS-Schwab classification or the GAP score is the most appropriate for the prediction of radiographic failure in patients suffering from ASD.

\section{MATERIALS AND METHODS}

The current study is a review of a two center cohort of patients suffering adult spinal deformity (ASD) who underwent posterior spinal fusion and instrumentation. Medical records and radiographs of patients with ASD treated with posterior spinal fusion between 2005 and 2015 at two spinal deformity centers in the Netherlands (Maastricht University Medical Center and Amsterdam University Medical Center) were retrospectively reviewed. Institutional review board (IRB) approval was obtained before study initiation (METC16-4-029) at each site. We included adults suffering ASD (defined as a deformity of the spine in either the coronal $\left(>10^{\circ}\right.$ lateral deviation of the vertical axis) or sagittal plane (T1 Pelvic Angle (TPA) $>10^{\circ}$ or T1 spinopelvic inclination (T1-SPi) angle > $\left.0^{\circ}\right)$ ), who received surgical treatment constituting posterior spinal fusion of at least four vertebrae, and of whom standing full spine plain radiographs pre- and postoperatively were available. Patients were excluded if less than four vertebrae were fused or if there was incomplete radiographic follow-up (less than 2 years of follow-up, or no full-spine radiographs).

All radiographs were analyzed using validated software (Surgimap, Nemaris Inc., New York, NY). Pelvic parameters that were measured were the Pelvic Incidence (PI), Pelvic Tilt (PT), and Sacral Slope (SS). Regional spinal parameters included PI-LL mismatch, L1-S1 Lumbar Lordosis (L1-S1 LL), L4-S1 Lumbar Lordosis (L4-S1 LL) and Thoracic Kyphosis (TK, T4-T12). Sagittal alignment was assessed linearly by T1 spinopelvic inclination (T1 Spi), T1 Pelvic Angle (TPA), Global Tilt (GT) and Global Sagittal Alignment (GSA) [18, 23-25]. 
The Global Alignment and Proportion (GAP) score comprises the Relative Pelvic Version (RPV), Relative Lumbar Lordosis (RLL), Lordosis Distribution Index (LDI), Relative Spinopelvic Alignment (RSA) and age [20]. The GAP score can range from 0 to 13 points. The cut-off points for the GAP score that were chosen were in accordance with the cut-off values as determined by Yilgor et al. [20]. RPV (measured sacral slope minus ideal sacral slope) of less than $-15^{\circ}$ was considered severe retroversion; $-15^{\circ}$ to $-7.1^{\circ}$, moderate retroversion; $-7^{\circ}$ to $5^{\circ}$, aligned; and more than $5^{\circ}$, anteversion. RLL (measured lumbar lordosis minus ideal lumbar lordosis) of less than $-25^{\circ}$ was considered severe hypolordosis; $-25^{\circ}$ to $-14.1^{\circ}$, moderate hypolordosis; $-14^{\circ}$ to $11^{\circ}$, aligned; and more than $11^{\circ}$, hyperlordosis. LDI (L4-S1 lordosis divided by L1-S1 lordosis multiplied by 100) of less than $40 \%$ was considered severe hypolordotic maldistribution; $40 \%$ to $49 \%$, moderate hypolordotic maldistribution; $50 \%$ to $80 \%$, aligned; and more than $80 \%$, hyperlordotic maldistribution. RSA (measured global tilt minus ideal global tilt) of more than $18^{\circ}$ was considered severe positive malalignment; $10.1^{\circ}$ to $18^{\circ}$, moderate positive malalignment; $10^{\circ}$ to $-7^{\circ}$, aligned; and less than $-7^{\circ}$, negative malalignment. A GAP score of 0 to 2 was categorized as indicating a proportioned spinopelvic state; 3 to 6 , as moderately disproportioned; more than 6, as severely disproportioned.

The original sagittal modifiers of the SRS-Schwab classification are Sagittal Vertical Axis (SVA), PT and PI-LL [19]. The SVA parameter is a distance and requires a calibrated image. Due to the fact that in the current study not all images were calibrated we used the T1-SPi for the assessment of global spinal alignment, which strongly correlates with SVA [23]. Patients with a T1-SPi of less than $1.35^{\circ}$ were classified with a T1 SPi modifier ' 0 ', a T1-SPi between $1.35^{\circ}$ and $7.2^{\circ}$ was classified with a T1-SPi modifier ' + ' and more than $7.2^{\circ}$ with a T1-SPi modifier '++'. Patients with a PI-LL value of less than $10^{\circ}$ were classified with a PI-LL modifier ' 0 ', a PI-LL value between $10^{\circ}$ and $20^{\circ}$ was classified with a PI-LL modifier '+' and greater than $20^{\circ}$ with a PI-LL modifier '++'. Patients with a PT of less than $20^{\circ}$ were classified with a PT modifier ' 0 ', a PT between $20^{\circ}$ and $30^{\circ}$ was classified with a PT modifier ' + ' and greater than $30^{\circ}$ with a PT modifier '++'. For statistical weight the following values were defined for the Schwab modifiers: 1 for modifier ' 0 ', 2 for modifier '+' and 3 for modifier ' ++ '.

The presence or absence of mechanical complications was defined as proximal junctional kyphosis or failure, distal junctional kyphosis or failure, rod breakage, and implant-related complications (Table 1) [20]. Revision surgery due to mechanical complications was defined as "mechanical revision". Based on these criteria three groups were defined: (1) "Normal” group (without mechanical complications), (2) "Non-revised" group (with radiographic mechanical complications, but without clinical indication for revision), (3) "Revised" group (with radiographic mechanical complications, patients underwent revision surgery). 
Table 1. Types and definitions of radiographic and implant-related mechanical complications used [17].

\begin{tabular}{ll}
\hline Type of mechanical complication & Definition \\
\hline Proximal junctional kyphosis (PJK) & $\begin{array}{l}\geq 10^{\circ} \text { increase in kyphosis between UIV and UIV+2 between } \\
\text { early postoperative and follow-up radiographs }\end{array}$ \\
Proximal junctional failure (PJF) & $\begin{array}{l}\text { Fracture of UIV or UIV+1, pullout of instrumentation at UIV, } \\
\text { and/or sagittal subluxation } \\
\geq 10^{\circ} \text { postoperative increase in kyphosis angle between LIV } \\
\text { and LIV-1 and/or pullout of instrumentation at LIV }\end{array}$ \\
Distal junctional kyphosis/failure & Single or double rod breakage \\
Rod breakage & $\begin{array}{l}\text { Other radiographic implant-related complications such as } \\
\text { screw loosening, breakage, or pullout or interbody graft, } \\
\text { Implant-related complications }\end{array}$ \\
& hook, or set-screw dislodgement
\end{tabular}

UIV: upper instrumented vertebra, LIV: lowest instrumented vertebra, +2 and $+1: 2$ and 1 vertebrae above UIV, and -1: 1 vertebra below LIV.

\subsection{Statistical Analysis}

We included all evaluable patients treated within the selected period of time. Baseline characteristics of the patients were described using mean and standard deviation (SD) for continuous variables, and as count and percentage for categorical variables. Patient characteristics in the three groups were compared using analysis of variance (ANOVA) for continuous variables, and the chi-square test for categorical variables.

ANOVA was used to determine if differences existed in radiographic parameters between the three groups and to compare the pre- and postoperative outcomes. We used logistic regression analysis to assess whether the GAP score or the Schwab classification was most appropriate for prediction of mechanical failure after dichotomization of the outcome. The ability of both the GAP score and the Schwab classification to discriminate between those who experienced mechanical failure, and those who did not was subsequently determined by computing the area under the Receiver Operating Characteristic (ROC) curve, or area under the curve (AUC), resulting from the logistic regression analysis. For this analysis the "Non failure" group was compared to the "Mechanical complication" group (comprising the "Non-revised" group and "Revised" group). In order to compare the ROC curves of the GAP score and the Schwab classification a nonparametric approach by deLong et al. [26] was used.

Correlations between the various factors constituting the GAP score and Schwab classification were estimated using the Spearman rank order correlation coefficient. All analyses were performed using $\mathrm{R}$ version 3.5.1. 


\section{RESULTS}

A total of 39 adult spinal deformity patients met the inclusion criteria and were included for analysis. The study population consisted of 10 men and 29 women, the average age was $60.18 \pm 9.67$ years (range, 39 to 82 years; Table 2). The "Normal" group was formed by 17 (44\%) patients, and radiographic mechanical complications were measured in 22 (56\%)

Table 2. Comparison of baseline demographics between groups.

\begin{tabular}{|c|c|c|c|c|c|}
\hline Variable & $\begin{array}{l}\text { Normal } \\
\text { group (1) }\end{array}$ & $\begin{array}{c}\text { Non-revised } \\
\text { group (2) }\end{array}$ & $\begin{array}{l}\text { Revised } \\
\text { group (3) }\end{array}$ & Total & $\begin{array}{l}\text { Chi-square } \\
\text { or ANOVA } \\
\text { (p-value for } \\
\text { difference) }\end{array}$ \\
\hline No. & 17 & 12 & 10 & 39 & \\
\hline Age (yrs) & $59.9 \pm 9.9$ & $61.4 \pm 8.8$ & $59.2 \pm 11.1$ & $60.2 \pm 9.7$ & $0.01(0.92)$ \\
\hline Female gender (no.) & $12(71 \%)$ & $11(92 \%)$ & $6(60 \%)$ & 29 (74\%) & $3.09(0.21)$ \\
\hline Prior spine surgery (no.) & $1(6 \%)$ & $0(0 \%)$ & $1(10 \%)$ & $2(5 \%)$ & $1.16(0.56)$ \\
\hline Diagnosis (no.) & & & & & $4.91(0.56)$ \\
\hline Degenerative & $13(76 \%)$ & $8(67 \%)$ & $9(90 \%)$ & $30(77 \%)$ & \\
\hline Idiopathic & $1(6 \%)$ & $0(0 \%)$ & $0(0 \%)$ & $1(3 \%)$ & \\
\hline Post-traumatic & $1(6 \%)$ & $3(25 \%)$ & $1(10 \%)$ & $5(13 \%)$ & \\
\hline Congenital & $2(12 \%)$ & $1(8 \%)$ & $0(0 \%)$ & $3(8 \%)$ & \\
\hline Mean no. of vertebrae fused & $7.7 \pm 2.8$ & $7.7 \pm 3.7$ & $7.3 \pm 2.0$ & $7.6 \pm 2.9$ & $0.11(0.74)$ \\
\hline
\end{tabular}

No statistically significant differences were found between the three groups for age, gender, prior spine surgery, diagnosis or mean number of vertebrae fused.

patients [12 (30\%) patients in the "Non-revised" group, 10 (26\%) patients in the "Revision" group]. No statistically significant differences were found between the three groups for age, gender, prior spine surgery, diagnosis or mean number of vertebrae fused (Table 2).

Preoperatively, there was no significant difference in GAP score and Schwab classification between the three groups ( $\mathrm{p}>0.05$ for all comparisons) (Figure $1 \mathrm{~A}$ and $\mathrm{B}$ ). For the "Normal" group the average GAP score and Schwab values declined postoperatively ( $\mathrm{p}=0.045$ and $\mathrm{p}=0.663$, respectively), whereas for the "Mechanical complication" groups these increased (Figure $1 \mathrm{~A}$ and $\mathrm{B} ; \mathrm{p}>0.05$ for all comparisons).

Postoperatively, the "Normal" group demonstrated significantly lower mean GAP scores in comparison to the "Non-revised" group and the "Revised" group ( $\mathrm{p}<0.001$ and $\mathrm{p}=0.005$, respectively) (Figure 2). The mean postoperative Schwab value for the "Normal" group was also significantly lower in comparison to the "Non-revised" group ( $\mathrm{p}=0.032$ ), whereas the difference between the "Normal" group and the "Revised" group was not significant ( $\mathrm{p}=0.250$; Figure 2 ). 
A.

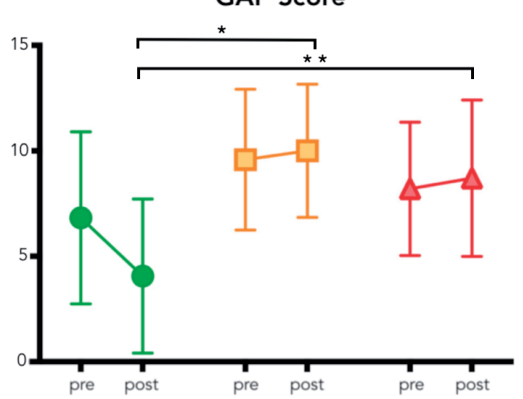

B. Schwab Classification

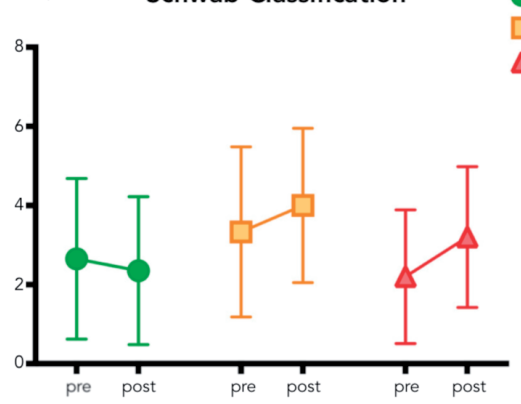

Figure 1. A. The mean pre- and postoperative GAP scores per group (mean \pm standard deviation, * indicates $\mathrm{p}<0.05)$. B. The mean pre- and postoperative values for the Schwab classification per group (mean \pm standard deviation, ${ }^{*}$ indicates $\mathrm{p}<0.05$ ).

Logistic regression analysis for the prediction of mechanical failure revealed a significant association with both the GAP score (OR 1.45, 95\% confidence interval [CI]: 1.16 - 1.79, $\mathrm{p}=0.001$ ) and the Schwab classification (OR 1.44, 95\% CI: $1.01-2.08, \mathrm{p}=0.046)$. The ability of both the GAP score and the Schwab classification to predict mechanical complications was expressed as the AUC. The AUC for the GAP score was 0.86 (95\% CI: $0.75-0.97$ ), whereas the AUC for the Schwab classification was 0.69 (95\% CI: $0.52-0.86)$. The difference between AUC's of the Schwab and the GAP score was statistically significant ( $\mathrm{p}=0.003$ ), indicating that the GAP discriminates better between those patients who will develop mechanical failure and those who will not. Subsequently, a plot of the predicted probability for the GAP score is visualized in Figure 3. For example, the risk of radio-

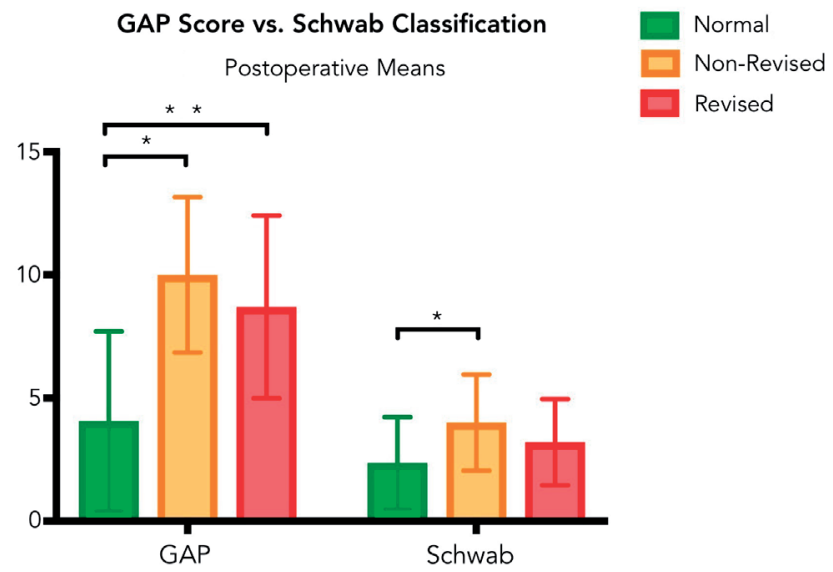

Figure 2. Postoperative mean values for the GAP score and Schwab classification per group (mean \pm standard deviation, ${ }^{*}$ indicates $\left.\mathrm{p}<0.05\right)$. 


\section{Risk Mechanical Complications}

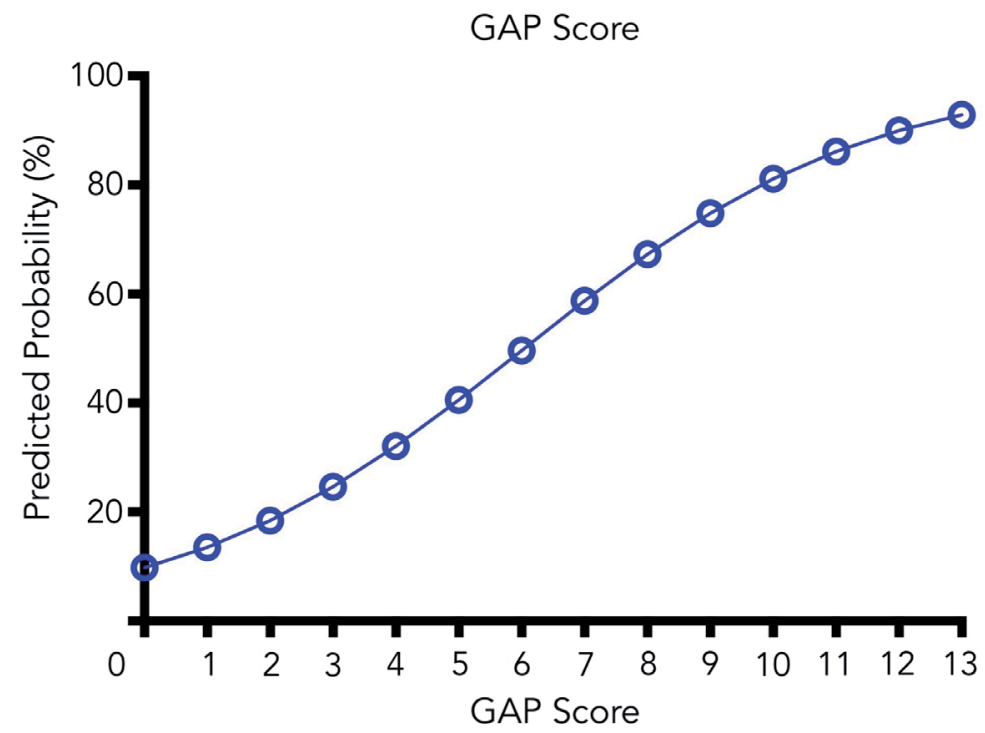

Figure 3. Predicted probability curve for the GAP score.

graphic mechanical complications for patients with a postoperative GAP score of 2 is approximately $18 \%$, whereas the risk is approximately $90 \%$ with a GAP score of 12 .

Spearman rank order correlation analysis showed that the GAP score was most strongly correlated with RSA, RLL and RPV $(\rho=0.85, \rho=0.84$, and $\rho=0.84$, respectively, $p<0.01$; Table 3). The correlation with the lordosis distribution index (LDI) was also significant but was as strong $(\rho=0.65, p<0.01)$. Age, on the contrary, showed poor correlation with the GAP score $(\rho=0.17, p=0.300)$. For the Schwab classification, the Spearman rank order correlation analysis showed a significant correlation with all three sagittal modifiers (PI-LL $\rho=0.90$, Global alignment (T1 SPi) $\rho=0.67$ and PT $\rho=0.70, p<0.01$ ), of which PI-LL was the strongest.

Table 3. Spearman rank order correlation coefficient analysis for the various determinants of the GAP score and the Schwab classification.

\begin{tabular}{lccc}
\hline & $\begin{array}{c}\text { Pelvic incidence minus } \\
\text { lumbar lordosis } \\
\text { (PI-LL) }\end{array}$ & $\begin{array}{c}\text { Global alignment } \\
\text { (T1 SPi) }\end{array}$ & $\begin{array}{c}\text { Pelvic tilt } \\
\text { (PT) }\end{array}$ \\
\hline Relative pelvic version (RPV) & $-0.69^{*}$ & -0.02 & $-0.84^{*}$ \\
Relative lumbar lordosis (RLL) & $-0.94^{\circ}$ & $-0.54^{\circ}$ & $-0.58^{*}$ \\
Lordosis distribution index (LDI) & 0.04 & 0.12 & $-0.32^{*}$ \\
Relative sagittal alignment (RSA) & $0.85^{\circ}$ & $0.55^{\circ}$ & $0.67^{*}$ \\
\hline
\end{tabular}

"indicate $\mathrm{p}<0.01$," indicate $\mathrm{p}<0.05$. 
The relative pelvic version (RPV) of the GAP score serves a similar role as the Pelvic Tilt (PT) modifier from the Schwab classification, demonstrating a significantly negative correlation coefficient $(\rho=-0.84, \mathrm{p}<0.01)$. In Table 4 , the distribution of patients over both subcategories (RPV and PT) is visualized, showing a few differences between the classification systems (e.g. more patients are aligned according to the GAP score than according to the Schwab modifiers). In Figure 4A the sacral slope is plotted against the pelvic incidence with respect to the "ideal sacral slope" [20]. The geometrical relationship between the pelvic angles and sacral slope should be kept in mind (pelvic incidence equals the sum of the sacral slope and pelvic tilt). Patients with a high pelvic incidence require a high pelvic tilt (and sacral slope), however according to the Schwab modifier these values are classified as "too high" ('+' or '++'). On the other hand, patients with a small pelvic incidence require an even smaller pelvic tilt and should be classified as ' ++ ' instead of '+'.

The relative lumbar lordosis (RLL) from the GAP score functions much like the PI-LL modifier from the Schwab classification and therefore also showed an extremely low correlation coefficient $(\rho=-0.94, p<0.01$ ). The distribution of patients over both subcategories (RLL and PI-LL) is visualized in Table 4 and appeared to be highly comparable. In Figure $4 \mathrm{~B}$ the lumbar lordosis is plotted against the pelvic incidence with respect to the "ideal lumbar lordosis" [20]. Again, the three groups presented are the three Schwab modifiers. Only for patients with pelvic incidence values near the upper-normal and lower-normal values the PI-LL shows limitations. Between a pelvic incidence of approximately 50 to 80 the PI-LL modifier is quite similar to the GAP score.

The relative spinal alignment (RSA) demonstrated a correlation coefficient $\rho=0.55(p<0.01)$ with the Global alignment (T1 SPi). The distribution of patients over both subcategories (RSA and Global alignment) is visualized in Table 4. According to the GAP score, approximately all patients show positive malalignment whereas according to the Schwab

Figure 4. A. Sacral slope (SS) per patient plotted against his/her pelvic incidence (PI) with respect to "ideal sacral slope" (green line). Green bold line represents alignment, upper orange and red areas represent anteversion, lower orange area represents moderate retroversion and lower red area represents severe retroversion. Symbols are coded according to the PT Schwab modifier ('0', ' + ' or '++', displayed as '*'). The blue boxes indicate differences between the GAP score and Schwab classification. Note: PI = PT + SS. B. Lumbar lordosis (LL) per patient plotted against his/her pelvic incidence (PI) with respect to "ideal lumbar lordosis" (green line). Green bold line represents alignment, upper orange and red areas represent hyperlordosis, lower orange area represents moderate hypolordosis and lower red area represents severe hypolordosis. Symbols are coded according to the PI-LL Schwab modifier ('0', '+' or '++', displayed as '*'). The blue boxes indicate differences between the GAP score and Schwab classification. C. Global tilt per patient plotted against his/her pelvic incidence (PI) with respect to "ideal global tilt" (green line). Green bold line represents alignment, upper orange area represents moderate positive malalignment, upper red area represents severe positive malalignment. Lower orange and red areas represent negative malalignment. Symbols are coded according to the PT Schwab modifier ('0', ' + ' or '++', displayed as '*'). The blue boxes indicate differences between the GAP score and Schwab classification. 


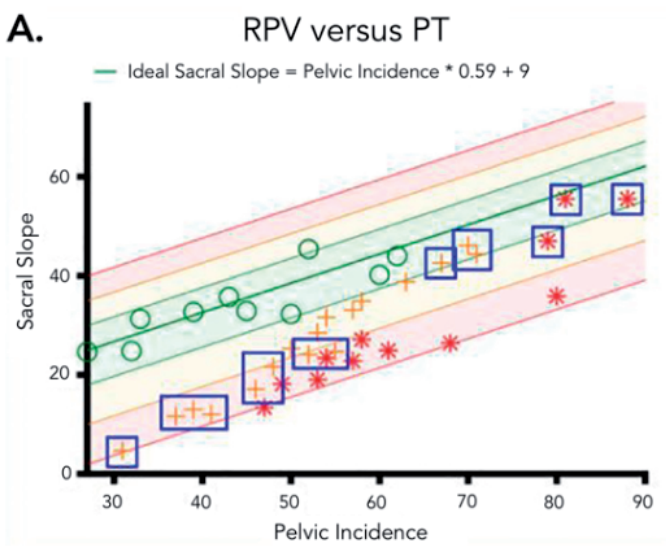

B.

RLL versus PI-LL

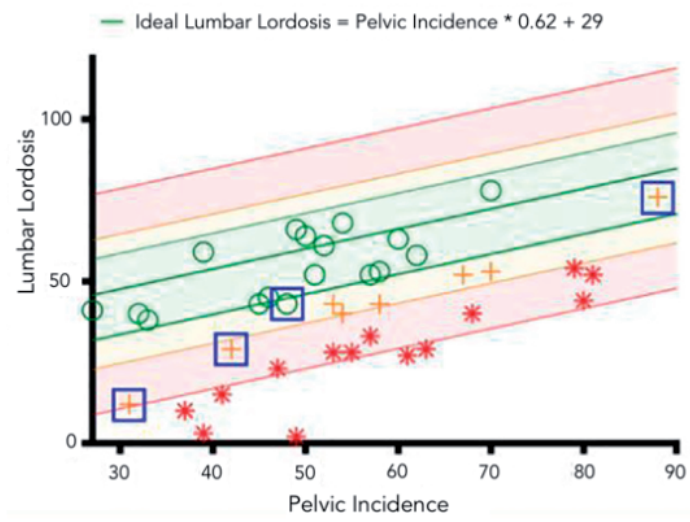

C. RSA versus Global Alignment

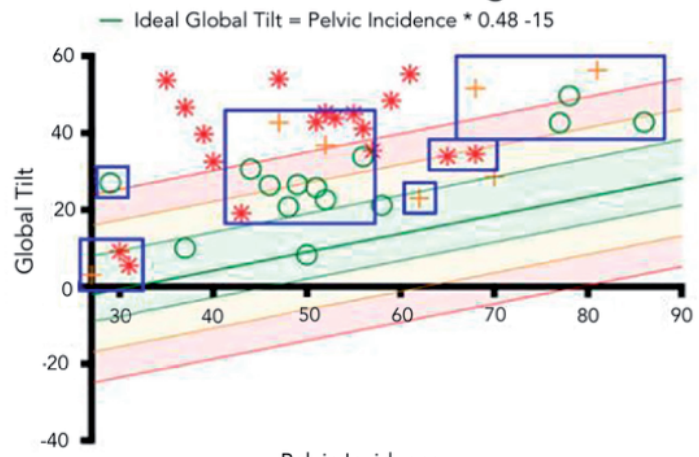

Pelvic Incidence

legend: $\bigcirc 0$ 米++ ++ 
Table 4. Distribution of patients over the various subcategories of the GAP score versus the Schwab classification (no. [\%]).

\begin{tabular}{|c|c|c|c|c|c|c|c|}
\hline GAP & Normal & $\begin{array}{l}\text { Non- } \\
\text { revised }\end{array}$ & Revised & Schwab & Normal & $\begin{array}{l}\text { Non- } \\
\text { revised }\end{array}$ & Revised \\
\hline \multicolumn{4}{|l|}{ Relative pelvic version } & \multicolumn{4}{|l|}{ Pelvic tilt } \\
\hline Anteversion & $\mathbf{1}(6)$ & $\mathbf{0}(0)$ & $\mathbf{0}(0)$ & 0 & $8(47)$ & $1(8)$ & $1(10)$ \\
\hline Aligned & $10(59)$ & $\mathbf{1}(8)$ & $2(20)$ & + & $5(29)$ & $5(42)$ & $7(70)$ \\
\hline Moderate retroversion & $2(12)$ & $2(17)$ & $3(30)$ & ++ & $4(24)$ & $6(50)$ & $2(20)$ \\
\hline Severe retroversion & $4(24)$ & $9(75)$ & $5(50)$ & & & & \\
\hline \multicolumn{4}{|l|}{ Relative lumbar lordosis } & PI-LL & & & \\
\hline Hyperlordotic & $\mathbf{0}(0)$ & $\mathbf{0}(0)$ & $\mathbf{0}(0)$ & 0 & $10(59)$ & $3(25)$ & $4(40)$ \\
\hline Aligned & $11(65)$ & $3(25)$ & $3(30)$ & + & $4(24)$ & $1(8)$ & $3(30)$ \\
\hline Moderate hypolordosis & $3(18)$ & $2(17)$ & $2(20)$ & ++ & $3(18)$ & $8(67)$ & $3(30)$ \\
\hline Severe hypolordosis & $3(18)$ & $7(58)$ & $5(50)$ & & & & \\
\hline \multicolumn{4}{|l|}{ Relative sagittal alignment } & \multicolumn{4}{|l|}{ Alignment } \\
\hline Negative & $\mathbf{0}(0)$ & $\mathbf{0}(0)$ & $\mathbf{0}(0)$ & 0 & $7(41)$ & $4(33)$ & $3(30)$ \\
\hline Aligned & $6(35)$ & $\mathbf{1}(8)$ & $\mathbf{0}(0)$ & + & $3(18)$ & $2(17)$ & $2(20)$ \\
\hline Moderately positive & $7(41)$ & $\mathbf{0}(0)$ & $2(20)$ & ++ & $7(41)$ & $6(50)$ & $5(50)$ \\
\hline Severely positive & $4(24)$ & $\mathbf{1 1}(92)$ & $8(80)$ & & & & \\
\hline \multicolumn{8}{|c|}{ Lordosis distribution index } \\
\hline Severely hypolordotic & $\mathbf{1}(6)$ & $\mathbf{1}(8)$ & $2(20)$ & & & & \\
\hline Moderate hypolordotic & $\mathbf{1}(6)$ & $2(17)$ & $\mathbf{0}(0)$ & & & & \\
\hline Aligned & $13(76)$ & $2(17)$ & $4(40)$ & & & & \\
\hline Hyperlordotic & $2(12)$ & $7(58)$ & $4(40)$ & & & & \\
\hline Total & & & & Total & & & \\
\hline Proportioned & $7(41)$ & $\mathbf{0}(0)$ & $\mathbf{0}(0)$ & 0 & $11(65)$ & $3(25)$ & $4(40)$ \\
\hline $\begin{array}{l}\text { Moderately } \\
\text { disproportioned }\end{array}$ & $6(35)$ & $\mathbf{1}(9)$ & $4(40)$ & + & $3(18)$ & $2(17)$ & $3(30)$ \\
\hline Severely disproportioned & $4(24)$ & $11(92)$ & $6(60)$ & ++ & $3(18)$ & $7(58)$ & $3(30)$ \\
\hline
\end{tabular}

classification the number of aligned patients is twice as high (this might explain the relatively low, but still significant, correlation coefficient) between RSA (GAP) and Global alignment (Schwab). In Figure 4C, the global tilt is plotted against the pelvic incidence with respect to the "ideal global tilt" [20] providing more insight in the differences found. These results indicate that the Schwab Classification might underestimate the malalignment with regard to a patient's individual pelvic incidence.

The distribution of patients over the two "novel" subcategories of the GAP score are visualized in Table 4. Hyperlordotic maldistribution appeared to be an important cause for radiographic mechanical complications. Age did not show to be a contributing factor to ability of the GAP score to predict mechanical complications. 


\section{DISCUSSION}

The current study aimed to assess which classification system, the SRS-Schwab classification or GAP score, is the most appropriate for the prediction of radiographic mechanical failure in patients operated for adult spinal deformity. The results demonstrated that both classification systems can predict radiographic mechanical failure, but the GAP score proved to be better with an AUC of 0.86 compared to 0.69 ( $\mathrm{p}=0.003$ ).

The outcome of the current study might explain why it is not uncommon to observe complications even after ideal correction of all sagittal modifiers from the Schwab classification [20]. A multicentre study by Soroceano et al. [22] found a prevalence of radiographic and implant-related complications of 31.7\% when using Schwab's target values (revision surgery was indicated in $52.6 \%$ of these patients). When the surgical goals according to the GAP score are achieved postoperatively, the shape of the spine allows for equal distribution of loads across the spinal column, pelvis and lower limbs. However, when the surgical goals according to the Schwab classification are achieved, this equilibrium might still be disrupted, which may lead to mechanical failure. There are several differences between the Schwab classification and the GAP score that might account for this [20]. For the Schwab classification the pelvic incidence minus lumbar lordosis, pelvic tilt and global alignment are independently used as numerical values $[19,21]$. On the contrary, for the GAP score all parameters are evaluated in relation to the pelvic incidence [27]. Pelvic incidence is a morphological parameter that remains constant during adulthood and is not affected by patient position or spinal surgery [28]. A patient with an upper-limit pelvic incidence will have a physiologically high pelvic tilt and sacral slope. According to the Schwab criteria the surgical goal in pelvic tilt for these patients is $<20^{\circ}$, however this is too low in case of a high PI and may actually increase the risk of mechanical complications instead of preventing them (Figure 4A: blue boxes on the right). For every patient the Schwab score advises a postoperative pelvic tilt of $<$ $20^{\circ}$, however for patients with a lower-limit pelvic incidence this is not accurate enough since these patients require values $<10^{\circ}$ (Figure $4 \mathrm{~A}$ : blue boxes on the left). Additionally, the PI-LL Schwab modifier does not apply for patients with extreme pelvic incidence values either (Figure 4B: blue boxes). Between a pelvic incidence of approximately 50 to 80 degrees the PI-LL modifier is quite accurate. Furthermore, since the Global Alignment (T1 SPi or SVA) is not regarded in relation to a patient's pelvic incidence, it is less accurate for the prediction of mechanical complications since it might underestimate the degree of malalignment (Figure 4C: blue boxes). These drawbacks underline the need for more patient-specific surgical thresholds as proposed by the GAP score.

A relatively novel subcategory of the GAP score is the lordosis distribution index (LDI), which divides the lumbar lordosis in a lower arc (L4-S1) and an upper arc (L1-L3). As described by Roussouly et al. [28], maldistribution between these arcs might alter the distribution of loads on the spinal column and might subsequently cause mechanical 
failure. The importance of this subcategory for the prediction of mechanical complications via the GAP score in the current study was highlighted by the significant correlation coefficient $(\rho=0.65, \mathrm{p}<0.01)$. Another addition to the GAP score was the subcategory "age". However, in the current study age was not an important determinant for the GAP score which is not in accordance with the literature [11]. Age, and especially age-related comorbidities (osteoporosis, sarcopenia, neurodegenerative factors), should always be considered during preoperative planning.

A good understanding of the principles of sagittal balance is vital to achieve optimum outcomes when treating adult spinal deformity. The major difference between the Schwab classification and the GAP score is the fact the GAP score is patient-tailored. Before the existence of the GAP score the aim of surgical treatment was to restore the spinal column to "normal values" (that applied for every patient) rather than patientspecific values. This might also explain why Smith et al. [18] found that complete correction of sagittal imbalance, based on an SVA of less than $50 \mathrm{~mm}$ postoperatively, was associated with a higher incidence of acute PJF. And why Glattes et al. showed that changes in sagittal vertical axis (SVA) did not contribute significantly to the development of abnormal PJK or PJF [15].

The current study was limited by a relatively small number of patients, although the differences that were observed were confirmed by statistical significance. Another limitation is that we had to substitute the SVA modifier of the Schwab classification by the T1 SPi. However, it was described by Lafage and Schwab [23] that the T1 SPi is strongly correlated with the SVA and therefore could be used when no calibrated radiographs are available.

It should be acknowledged that deformity surgery is a challenging and complex procedure with high reported complication rates. Surgical complications encompass a variety of entities which cannot all be predicted by means of radiographs (e.g. neurological deficits, incidental durotomy and wound dehiscence). Furthermore, it should be taken into account that inadequate (pre- or postoperative) spinal alignment is not the only risk factor for complications. Older age, high body mass index and low bone mineral density are important risk factors which should be taken into consideration when planning surgical treatment [12-15].

In conclusion, the ability to predict radiographic mechanical complications following adult spinal deformity surgery by the Schwab classification and the GAP score was studied. Both classification systems were able to predict mechanical complications, however the GAP score proved to be significantly more appropriate. The difference found is probably attributed to the fact that in the GAP score all parameters are related to the patient's individual pelvic incidence. 


\section{REFERENCES}

1. Barrey C, et al. Sagittal balance disorders in severe degenerative spine. Can we identify the compensatory mechanisms? Eur Spine J, 2011. 20 Suppl 5: p. 626-33.

2. Glassman SD, et al. The impact of positive sagittal balance in adult spinal deformity. Spine (Phila Pa 1976), 2005. 30(18): p. 2024-9.

3. Diebo BG, et al. Sagittal deformities of the spine: factors influencing the outcomes and complications. Eur Spine J, 2015. 24 Suppl 1: p. S3-15.

4. Yamada K, et al. Targeted Therapy for Low Back Pain in Elderly Degenerative Lumbar Scoliosis: A Cohort Study. Spine (Phila Pa 1976), 2016. 41(10): p. 872-9.

5. Takahashi S, J Delecrin and N. Passuti. Surgical treatment of idiopathic scoliosis in adults: an age-related analysis of outcome. Spine (Phila Pa 1976), 2002. 27(16): p. 1742-8.

6. Shapiro GS, G Taira and O Boachie-Adjei. Results of surgical treatment of adult idiopathic scoliosis with low back pain and spinal stenosis: a study of long-term clinical radiographic outcomes. Spine (Phila Pa 1976), 2003. 28(4): p. 358-63.

7. Schwab FJ, et al. Risk factors for major peri-operative complications in adult spinal deformity surgery: a multi-center review of 953 consecutive patients. Eur Spine J, 2012. 21(12): p. 2603-10.

8. Bianco K, et al. Complications and intercenter variability of three-column osteotomies for spinal deformity surgery: a retrospective review of 423 patients. Neurosurg Focus, 2014. 36(5): p. E18.

9. Pichelmann MA, et al. Revision rates following primary adult spinal deformity surgery: six hundred forty-three consecutive patients followed-up to twenty-two years postoperative. Spine (Phila Pa 1976), 2010. 35(2): p. 219-26.

10. Hostin R, et al. Incidence, mode, and location of acute proximal junctional failures after surgical treatment of adult spinal deformity. Spine (Phila Pa 1976), 2013. 38(12): p. 1008-15.

11. Lau D, et al. Proximal junctional kyphosis and failure after spinal deformity surgery: a systematic review of the literature as a background to classification development. Spine (Phila Pa 1976), 2014. 39(25): p. 2093-102.

12. Yagi $\mathrm{M}$, et al. Characterization and surgical outcomes of proximal junctional failure in surgically treated patients with adult spinal deformity. Spine (Phila Pa 1976), 2014. 39(10): p. E607-14.

13. O'Leary PT, et al. Risk factors and outcomes for catastrophic failures at the top of long pedicle screw constructs: a matched cohort analysis performed at a single center. Spine (Phila Pa 1976), 2009. 34(20): p. 2134-9.

14. Kim HJ, et al. Patients with proximal junctional kyphosis requiring revision surgery have higher postoperative lumbar lordosis and larger sagittal balance corrections. Spine (Phila Pa 1976), 2014. 39(9): p. E576-80.

15. Bridwell KH, et al. Proximal junctional kyphosis in primary adult deformity surgery: evaluation of 20 degrees as a critical angle. Neurosurgery, 2013. 72(6): p. 899-906.

16. Bridwell $\mathrm{KH}$, et al. Changes in radiographic and clinical outcomes with primary treatment adult spinal deformity surgeries from two years to three- to five-years follow-up. Spine (Phila Pa 1976), 2010. 35(20): p. 1849-54.

17. Cho KJ, et al. Risk factors of sagittal decompensation after long posterior instrumentation and fusion for degenerative lumbar scoliosis. Spine (Phila Pa 1976), 2010. 35(17): p. 1595-601. 
18. Smith MW, et al. Acute proximal junctional failure in patients with preoperative sagittal imbalance. Spine J, 2015. 15(10): p. 2142-8.

19. Schwab F, et al. Scoliosis Research Society-Schwab adult spinal deformity classification: a validation study. Spine (Phila Pa 1976), 2012. 37(12): p. 1077-82.

20. Yilgor C, et al. Global Alignment and Proportion (GAP) Score: Development and Validation of a New Method of Analyzing Spinopelvic Alignment to Predict Mechanical Complications After Adult Spinal Deformity Surgery. J Bone Joint Surg Am, 2017. 99(19): p. 1661-1672.

21. Schwab FJ, et al. Adult scoliosis: a quantitative radiographic and clinical analysis. Spine (Phila Pa 1976), 2002. 27(4): p. 387-92.

22. Soroceanu A, et al. Radiographical and Implant-Related Complications in Adult Spinal Deformity Surgery: Incidence, Patient Risk Factors, and Impact on Health-Related Quality of Life. Spine (Phila Pa 1976), 2015. 40(18): p. 1414-21.

23. Lafage $\mathrm{V}$, et al. Pelvic tilt and truncal inclination: two key radiographic parameters in the setting of adults with spinal deformity. Spine (Phila Pa 1976), 2009. 34(17): p. E599-606.

24. Protopsaltis T, et al. TheT1 pelvic angle, a novel radiographic measure of global sagittal deformity, accounts for both spinal inclination and pelvic tilt and correlates with health-related quality of life. J Bone Joint Surg Am, 2014. 96(19): p. 1631-40.

25. Obeid I, et al. The Global Tilt: Evaluation of a Parameter Considering the Global Spinopelvic Alignment. J Med Liban, 2016. 64(3): p. 146-51.

26. DeLong ER, DM DeLong and DL Clarke-Pearson. Comparing the areas under two or more correlated receiver operating characteristic curves: a nonparametric approach. Biometrics, 1988. 44(3): p. 837-45.

27. Obeid I, et al. Global tilt: a single parameter incorporating spinal and pelvic sagittal parameters and least affected by patient positioning. Eur Spine J, 2016. 25(11): p. 3644-3649.

28. Roussouly P and C Nnadi. Sagittal plane deformity: an overview of interpretation and management. Eur Spine J, 2010. 19(11): p. 1824-36. 




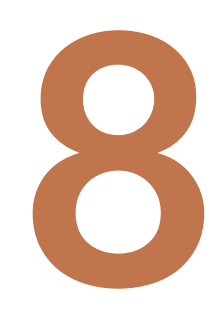

\section{General Discussion}

Valorization 



\section{GENERAL DISCUSSION}

This thesis focused on increasingly important aspects of spinal aging. In this final chapter, the main findings and limitations of the previous chapters are discussed, and general conclusions and recommendations as well as directions for future research are provided.

In Chapter 2, we elaborated on the spatiotemporal gait parameters and their variability in postmenopausal women with and without vertebral fractures. It was found that patients suffering an osteoporotic vertebral compression fracture (OVCF) walk with significantly shorter and wider strides as compared to their healthy counterparts. Six months after fracture the majority of the spatiotemporal gait parameters (e.g. stability, double support time and step width) have only partially improved. This was the first study to elucidate the spatiotemporal gait characteristics and their variability in postmenopausal women suffering an OVCF in comparison to healthy subjects over time, which obviously limits comparison with previous studies.

Gait is an important indicator of health and is a strong predictor of future limitations in mobility and falls among older adults $[1,2]$. The cautious gait pattern found in the OVCF group, typically marked by mild to moderate slowing, reduced stride length, and widening of the base of support, has been associated with an increased risk of falling $[3,4]$. The origin of the cautious gait is not well understood, however, pain and fear of falling presumably play an important role [5]. Older adults may increase their double support time, which is a stabilizing factor during a normal gait cycle, and increase their step width in order to stabilize their inefficient gait control and prevent falls [6]. Falling is not only associated with increased morbidity and mortality in older individuals, but is also linked to poor overall functioning and early admission to long-term care facilities [3]. Reducing the risk of falling in the older population is therefore an important public health objective. Our results are in accordance with literature [7], and imply that after suffering an OVCF patients are at a greater risk of incident disability, such as falling, than their healthy counterparts, even six months after conservative treatment. These results underline that an OVCF can be the serious beginning of a downward cascade which should be taken into account in the multimodal treatment of vertebral compression fractures and osteoporosis (Figure 3, Chapter 1) [7, 8]. Most patients with an OVCF are currently treated conservatively with analgesics, bracing, early rehabilitation and osteoporosis treatment with bisphosphonates [9]. However, in the current treatment algorithms too little attention is paid to the prevention of falling $[9,10]$. The American Geriatrics Society and British Geriatrics Society recommend that all adults older than 65 years should be screened annually for a history of falls or balance impairment [3, 11]. Subsequently, older adults who are at an increased risk of falling should be prescribed multifactorial programs, which include aerobic exercise supplemented with step, balance, strength and gait training to prevent falls [11]. A meta-analysis by Okubu et al.[12] demonstrated that step and balance training can prevent falls by approximately $50 \%$ in 
older adults due to improvements in balance recovery, reaction time, gait and balance. In comparison to general training during physical therapy, step training provided a greater fall reduction effect due to the greater task specificity in which the neurophysiological and sensorimotor skills required to prevent falls are trained [12]. Because of the fact that patients do not fully recover to their previous level of mobility after six months of conservative treatment, it appears to be of high importance to add balance and gait training to the treatment algorithm of OVCFs.

After an OVCF, the risk of a new vertebral fracture in the subsequent year is approximately $20 \%$ [13]. Baek et al. [14] demonstrated that in osteoporotic patients, the most important risk factors for novel vertebral compression fractures after the initial fracture are the altered biomechanics of the spine due to sagittal malalignment and of course the degree of osteoporosis. Treatment of OVCFs should aim to break the downward spiral of recurrent vertebral compression fractures and to prevent or slowdown the decline in postural control and the subsequent progression of hyperkyphosis with resultant global sagittal malalignment and imbalance (Figure 3, Chapter 1) [15]. In order to minimize increase of load on the anterior part of the spine (vertebral bodies) and prevent new vertebral fractures, prevention of an increased anterior bending moment on the trunk seems of high clinical importance [16]. In Chapter 3, the value of a semirigid thoracolumbar orthosis on gait and sagittal alignment was evaluated. It was shown that six weeks of continuous dynamic bracing results in a more upright posture and adjusted gait pattern in female patients suffering an OVCF. This is clinically relevant since even a small increase in thoracic kyphosis will result in a significant increase in vertebral compressive loading (Figure 4, Chapter 1) [17]. The reduced biomechanical strength of the anterior column in patients suffering osteoporosis, combined with the compressive overload may cause progressive collapse of the unsupported anterior cortex resulting in novel vertebral fractures $[18,19]$. Vertebral compression fractures are approximately five- to tenfold more prevalent in patients with moderate or severe kyphosis of the thoracolumbar spine in comparison to patients with mild kyphosis [16]. Therefore, in order to minimize increase in spinal loading and prevent new fractures, prevention of an increased thoracolumbar kyphosis is key in patients suffering an osteoporotic vertebral compression fracture. Moreover, it was shown by Miyakoshi et al. [20], that radiographic improvement of sagittal alignment in osteoporotic patients with fractures improved their quality of life. Various methods of decreasing the load on the anterior part of the spine have been proposed and positive effects of exercise programs, training of the back extensor muscles and posture training on pain, quality of life, reduction of the kyphosis and the risk of future vertebral fractures have been found [21-23]. Although the role of dynamic bracing in the treatment of OVCFs is controversial [10, 24], Pfeifer et al. [25, 26] demonstrated an increase in back extensor strength, improved posture, improved pulmonary function and positive effects on pain and activities of daily life. These results are in accordance with the results we found in Chapter 3. A recent study by Kaijser et al. [23] 
compared the effect of treatment with dynamic bracing to physical, equipment training and to a control group in patients suffering from osteoporosis with or without a vertebral fracture. Wearing an activating spinal orthosis (dynamic bracing) for at least two hours a day for six months demonstrated no significant difference in back pain, back extensor strength, and kyphotic index compared to the other groups. However, back extensor strength increased by $27 \%$ percent, which may be very important to prevent progression of sagittal imbalance [22, 23, 27]. Nevertheless, a large randomized controlled trial will be required to investigate the efficacy, in terms of pain reduction, and the effect of dynamic bracing on gait and posture in relation to the natural healing process of the fractured vertebra. Follow-up of two to five years will be required to further investigate whether the more upright posture will prevent the development of hyperkyphosis and actually cause a decrease in new vertebral fractures.

Although most osteoporotic vertebral compression fractures heal within a few months, a substantial part of the patients fail to respond to conservative treatment and often require long-term care [28]. Venmans et al. [29] found that $60 \%$ of conservatively treated patients with acute OVCFs had sufficient pain relief and good functional recovery within approximately three months after the acute fracture. However, $40 \%$ of patients still had disabling pain after one year [29, 30]. In a study by Zhang et al. [28], risk factors for conservative treatment failure were identified. Increased age, decreased bone mineral density, increased body mass index, and a high modified frailty index were high-risk factors for failure of conservative treatment of acute OVCFs [28]. For patients with high-risk factors active observation is highly recommended during conservative treatment and early-stage treatment policy change should be considered (within a few weeks of fracture) [28, 31]. According to the Vertos IV study [32], there is a place for vertebroplasty for these fractures that are not responsive to conservative treatment when the efficacy outweighs the risks. The currently used bone cements in vertebroplasty are derived from conventional polymethylmethacrylate (PMMA) bone cements used in total hip and knee arthroplasties. The main modification is that, for safety reasons, cements used for vertebroplasty should be clearly visible on fluoroscopy and thus contain a much higher proportion (up to $30 \%$ on weight basis) of radiopacifier (most commonly barium sulphate $\left(\mathrm{BaSO}_{4}\right)$ ) [33]. However, there are a number of drawbacks to mixing PMMA with large concentrations of $\mathrm{BaSO}_{4}$. These include adverse effects on injectability, viscosity profile, setting time, mechanical properties of the cement and bone resorption by macrophage activation. In Chapter 4, a novel PMMA cement was introduced, that has been designed to address these drawbacks. Its powder includes PMMA microspheres in which gold particles are incorporated for radiopacity, which is mixed with the usual monomer from commercial cements for vertebroplasty. In comparison to one such commercial cement brand (VertaPlex ${ }^{\mathrm{TM}}$ ) the new cement has longer doughing time, longer injection time, higher compressive strength, higher compressive modulus, and is less cytotoxic. Cement injection into human cadaveric vertebrae with simulated compression fractures 
revealed that the mechanical and filling properties of the gold-containing microsphere cement were not compromised relative to the commercial cement. The results found in Chapter 4 indicate that there are several possibilities to optimize the cement used for vertebroplasty with positive effects on injectability, viscosity profile, setting time, mechanical properties and bone resorption. For example, another well-known drawback of vertebroplasty is the increased risk of (re)collapse of the cemented or adjacent vertebrae due to excessive stiffness of the cement [34-36]. The strength and stiffness of individual fractured vertebral bodies is increased by cement augmentation to values matching or exceeding those of intact vertebrae. Increased stresses and strains in the adjacent vertebrae, resulting from the filling of the vertebral body with bone cement, may cause an alteration of the load transfer [36]. Theoretically, the stress on the augmented and adjacent vertebral bodies could be reduced by lowering the elastic modulus of bone cement, thereby decreasing the occurrence of adjacent vertebral fractures [34]. Future research in this field is of high clinical importance since recurrent vertebral compression fractures may subsequently progress to increased thoracolumbar kyphosis and resultant risks of new fractures and global sagittal malalignment [8, 37].

Global sagittal malalignment in Adult Spinal Deformity (ASD) can cause severe pain, disability and progressive neurological deficit. In the absence of rapid progressive curves, concerning instability or progressive neurological deficits conservative management is preferred. Nonoperative treatment typically consists of physical therapy focusing on low-impact core strengthening and endurance, analgesics, bracing, epidural steroid injections, selective nerve root blocks and/or facet blocks [38]. Spinal fusion using rigid instrumentation is currently the salvage treatment to stop further curve progression after conservative treatment has failed $[39,40]$. However, the mechanical stiffness mismatch between rigid spinal instrumentation and low mineral density bone in case of osteoporosis has been suggested to contribute to high mechanical failure rates in ASD surgery [40-42]. In Chapter 5, the effect of variation in rod stiffness (titanium versus polycarbonate-urethane (PCU)) on the loading of the anterior spinal column, as measured by intradiscal pressure (IDP) of fixed spinal segments during flexion-compression, was assessed. Low implant stiffness was found to generate lower IDP during flexioncompression loading in comparison to high implant stiffness (almost similar to the uninstrumented control group), representing a more physiological loading pattern. The use of dynamic spinal implants or posterior based motion preserving systems has extensively been reviewed in biomechanical studies [43, 44]. Dynamic spinal implants, based on pedicle screw fixation coupled with a flexible longitudinal connecting system, were invented to prevent adjacent segment disease after fusion surgery [45, 46]. However, long-term clinical studies showed that dynamic spinal implants do not have a clinical advantage over fusion surgery in the prevention of adjacent segment disease after short segment instrumentation [47, 48]. A plausible explanation is the absence of bony fusion, which leads to micromotion and formation of fibrous tissue around the screws causing 
screw pull-out and junctional failure [49]. These results suggest that caution is warranted when using polycarbonate-urethane (PCU) rods as standalone fixation (e.g. substituting the rigid titanium rods) in the surgical treatment of ASD [43, 44]. The "topping-off" concept, however, may be an alternative to overcome the gap between rigid and dynamic spinal fixation. In topping-off, spinal fusion is combined with dynamic stabilization of the cranial adjacent segment. This technique provides a more gradual transition of forces between the rigidly fused construct and the adjacent mobile segments [50]. The results of the concept study presented in Chapter $\mathbf{5}$ indicate that PCU could be a feasible material for topping-off. The low and tuneable implant stiffness of PCU would allow for a more physiological load distribution, with the aim of minimizing the load transfer and subsequent stresses at the bone-screw interface and the possible benefit of a decrease in adjacent segment disease.

As mentioned before, surgery for ASD is a challenging and complex procedure with high reported complication (8.4-42\%) and revision rates (9-17.6\%) [51, 52]. The high complication rates warrant the assessment of individual risk profiles and a patient-tailored treatment plan for patients suffering ASD, either surgical or non-surgical. Treatment for ASD is characterized by a substantial variety in treatment advice as there is no generally acknowledged consensus for decision-making. In an attempt to improve evidence-based clinical decision-making and increase uniformity and transparency of care, the Scoliosis Research Society (SRS) established appropriateness criteria for surgery for DLS [53]. These criteria comprise seven clinical or radiographic characteristics: (1) severity of selfreported symptoms, (2) severity of central spinal or foraminal stenosis, (3) progression of the degree of curvature or certain other radiographic abnormalities, (4) presence of sagittal imbalance, (5) severity of risk factors for suboptimal outcomes, (6) degree of curvature, and (7) when applicable, the number of levels with at least moderate central spinal or foraminal stenosis. Based on these characteristics it is determined for each patient whether or not surgery is inappropriate, appropriate, or necessary. In these criteria, however, the patient perspective has not been formally incorporated, although it is the patient's experienced burden of disease, combined with the surgeon's recommendation that will eventually determine the decision-making process. Informed choice includes both patient preferences and evidence-based clinical recommendations. Since patient perspective is an increasingly important consideration in informed decisionmaking, embedding Patient Reported Outcome Measures (PROMs) in the appropriateness criteria may allow for a more uniform and transparent patient-centered approach [53]. In Chapter 6 the extent to which the patient perspective was integrated into the appropriateness criteria of surgery for DLS was evaluated. It was found that there was a significant association between the appropriateness of surgery and Patient Reported Outcome Measures (PROMs). The discriminative ability for appropriateness of surgery for PROMs as a group was strong (AUC of 0.83). However, when considered in isolation, the predictive power of any individual PROM was poor. The different categories of the ap- 
propriateness criteria significantly coincided with the PROMs used. The results found in Chapter 6 are comparable with those from Bess et al. [54] and Richner-Wunderlin et al. [55] who both stated that pain and disability should determine the treatment modality in older patients, whereas in the contrary in younger patients it should be determined by the degree of deformity. Implementation of PROMs into the appropriateness criteria may be a possibility to allow for a more transparent, quantifiable and uniform clinical decision-making process.

For specific ASD patients surgical intervention can offer superior clinical and radiographic outcomes compared with nonoperative approaches, however, with a high risk of complications [51, 52]. The main cause of mechanical complications after ASD surgery is failure to achieve or maintain adequate postoperative sagittal alignment. In order to define appropriate surgical targets, the Scoliosis Research Society (SRS)-Schwab classification and the Global Alignment and Proportion (GAP) score were established [56, 57]. In Chapter 7, we assessed and compared the ability of the Schwab classification and the GAP score to predict mechanical complications following adult spinal deformity surgery. The results demonstrated that both classification systems are capable of predicting radiographic evidence of mechanical failure, however the GAP score proved to be significantly better $(\mathrm{p}=0.003$ ). This difference is probably attributed to the fact that in the GAP score all parameters are related to the patient's individual pelvic incidence. It is important to not just restore the spinal column to "normal values" but to restore it to patient-specific values. Moreover, it should be taken into account that inadequate spinal alignment is not the only risk factor for complications. Older age, high body mass index and low bone mineral density are also important risk factors which should be taken into consideration when planning surgical treatment for ASD [58-60]. Personalized medicine is of increasing importance for patients suffering from ASD, as they constitute a heterogeneous patient population with diverse clinical presentations, treatment indications as well as treatment outcomes.

\section{CONCLUSION AND RECOMMENDATIONS}

With aging of the population, clinicians will be required to manage an increasing number of spinal disorders specific to the elderly, which poses us for important challenges in treatment because this patient category is associated with multiple medical comorbidities, decreased mobility, poor balance, and a greater propensity to falling. When designing and implementing therapeutic strategies, clinicians must consider all of these factors to ensure adequate patient support, optimize outcomes and prevent catastrophic events.

Based on the results of this thesis the following recommendations can be made: 
- After an osteoporotic vertebral compression fracture (OVCF) patients do not recover to their previous level of mobility, to this end balance and gait training should be structurally included in the treatment algorithm of OVCFs (Chapter 2);

- Dynamic bracing for the treatment of OVCFs could prevent or slowdown the decline in postural control and the subsequent progression of hyperkyphosis with resultant global sagittal malalignment and imbalance. However, a large randomized controlled trial will be necessary for definite proof of efficacy in terms of pain relief and postural control (Chapter 3);

- Gold-containing microsphere polymethylmethacrylate (PMMA) cement is an interesting modifiable alternative to the commercial PMMA bone cements with positive effects on injectability, viscosity profile, setting time, mechanical properties and bone resorption (Chapter 4);

- Polycarbonate-urethane (PCU) could be a feasible material for topping-off in adult spinal deformity surgery, as it allows for a more physiological load distribution to the adjacent segments (Chapter 5);

- The implementation of PROMs into the appropriateness criteria for degenerative lumbar scoliosis surgery allows for a more patient-centered, transparent and uniform clinical decision-making process (Chapter 6);

- When performing surgery for adult spinal deformity the Global Alignment and Proportion (GAP) score is the most appropriate tool for surgical planning (Chapter 7).

\section{FUTURE PERSPECTIVES}

Treatment for osteoporotic vertebral compression fractures (OVCFs) is characterized by a substantial variety in treatment advice as there is no generally acknowledged consensus for decision-making. Therapeutic recommendations for conservative treatment are generally inconsistent [61]. Since OVCFs are an increasing health problem with serious clinical consequences, high-quality studies on the management of OVCFs are warranted. The most important direction for future research would be to conduct a large, randomized, blinded controlled trial to determine which treatment pathways are (cost)effective and safe for patients. Based on the results found in this thesis balance training and dynamic bracing should be taken into consideration in the treatment algorithm.

The current understanding of the impact of surgical correction of spinal deformity on the functional mobility and daily activities of patients is incomplete. Spinal alignment is a postural concept, as currently evaluated on static standing radiographs, that serves as the basis for the preoperative and postoperative assessment of patients with spinal deformity [62]. However, spinal balance and body balance are active processes that require a thorough understanding of the harmony between posture, balance and motion, which is not be fully represented by static radiographs alone. Three-dimensional motion 
analysis can bridge the gap between static spinal radiographic alignment, physiologic dynamic body balance and gait in the setting of adult spinal deformity. The Computer Assisted Rehabilitation Environment (CAREN) is an acknowledged tool to assess balance and motion/gait in patients. The system is able to provide a self-paced mode in which the patient is able to walk at a self-selected speed on a treadmill. The system allows continuous gait assessment in comparison to a normal gait lab, allowing more data to be collected and the outcomes in the end to be more reliable. With the CAREN system perturbations can be created to challenge dynamic postural stability in order to better mimic daily life motion. Thus, the CAREN system provides a novel and more representative method to study human posture and balance while walking. Therefore, the CAREN system could be an efficient tool in the objectivation of spinal alignment during ambulation and other activities of daily living before and after surgical treatment for adult spinal deformity. Motion analysis can provide insight into deficiencies in gait and balance, as seen in patients with adult spinal deformity, and has the ultimate potential by analyzing gait patterns, to help determine and monitor proper intervention to restore dynamic balance. Thus, dynamic alignment data may allow for a more individualized and appropriate surgical treatment. To this end, analysis using the CAREN system has currently been implemented in our standard patient care for adult spinal deformity. The purpose of our future studies is to evaluate the effect of long segment spinal fusion on static and dynamic spinal balance in adult spinal deformity patients. The objective measures obtained with the CAREN system will then be correlated to radiographic evaluations and patient reported outcomes pertaining to pain, function and quality of life. Gait analysis in patients with adult spinal deformity have the potential to improve our understanding of dynamic compensatory mechanisms, the hip-spine complex, and proximal junctional kyphosis. Dynamic and functional assessments such as gait analysis are the future of adult spinal deformity research, with potential clinical and surgical applications. 


\section{REFERENCES}

1. Hausdorff JM. Gait variability: methods, modeling and meaning. J Neuroeng Rehabil, 2005. 2: p. 19.

2. Verghese J. et al. Quantitative gait markers and incident fall risk in older adults. J Gerontol A Biol Sci Med Sci, 2009. 64(8): p. 896-901.

3. Guideline for the prevention of falls in older persons. American Geriatrics Society, British Geriatrics Society, and American Academy of Orthopaedic Surgeons Panel on Falls Prevention. J Am Geriatr Soc, 2001. 49(5): p. 664-72.

4. Nutt JG. Classification of gait and balance disorders. Adv Neurol, 2001. 87: p. 135-41.

5. Herman T, et al. Gait instability and fractal dynamics of older adults with a "cautious" gait: why do certain older adults walk fearfully? Gait Posture, 2005. 21(2): p. 178-85.

6. Kwon MS, et al. Comparison of gait patterns in elderly fallers and non-fallers. Technol Health Care, 2018. 26(S1): p. 427-436.

7. de Groot $\mathrm{MH}$, et al. Testing postural control among various osteoporotic patient groups: a literature review. Geriatr Gerontol Int, 2012. 12(4): p. 573-85.

8. Broy SB. The Vertebral Fracture Cascade: Etiology and Clinical Implications. J Clin Densitom, 2016. 19(1): p. 29-34.

9. Luthman S, J Widen and F Borgstrom. Appropriateness criteria for treatment of osteoporotic vertebral compression fractures. Osteoporos Int, 2018. 29(4): p. 793-804.

10. Rzewuska M, et al. The efficacy of conservative treatment of osteoporotic compression fractures on acute pain relief: a systematic review with meta-analysis. Eur Spine J, 2015. 24(4): p. 702-14.

11. Moncada LVV and LG Mire. Preventing Falls in Older Persons. Am Fam Physician, 2017. 96(4): p. 240-247.

12. Okubo Y, D Schoene and SR Lord. Step training improves reaction time, gait and balance and reduces falls in older people: a systematic review and meta-analysis. Br J Sports Med, 2017. 51(7): p. 586-593.

13. Lindsay R, et al. Risk of new vertebral fracture in the year following a fracture. JAMA, 2001. 285(3): p. 320-3.

14. Baek SW, C Kim and H Chang. The relationship between the spinopelvic balance and the incidence of adjacent vertebral fractures following percutaneous vertebroplasty. Osteoporos Int, 2015. 26(5): p. 1507-13.

15. van der Jagt-Willems HC, et al. Associations between vertebral fractures, increased thoracic kyphosis, a flexed posture and falls in older adults: a prospective cohort study. BMC Geriatr, 2015. 15: p. 34.

16. Wei Y, et al. Thoracolumbar kyphosis is associated with compressive vertebral fracture in postmenopausal women. Osteoporos Int, 2017. 28(6): p. 1925-1929.

17. Bruno AG, et al. The effect of thoracic kyphosis and sagittal plane alignment on vertebral compressive loading. J Bone Miner Res, 2012. 27(10): p. 2144-51.

18. Hou Y and Z Luo. A study on the structural properties of the lumbar endplate: histological structure, the effect of bone density, and spinal level. Spine (Phila Pa 1976), 2009. 34(12): p. E427-33.

19. Landham PR, et al. Pathogenesis of Vertebral Anterior Wedge Deformity: A 2-Stage Process? Spine (Phila Pa 1976), 2015. 40(12): p. 902-8. 
20. Miyakoshi $\mathrm{N}$, et al. Improvement of spinal alignment and quality of life after corrective surgery for spinal kyphosis in patients with osteoporosis: a comparative study with nonoperated patients. Osteoporos Int, 2015. 26(11): p. 2657-64.

21. Sinaki M, et al. Stronger back muscles reduce the incidence of vertebral fractures: a prospective 10 year follow-up of postmenopausal women. Bone, 2002. 30(6): p. 836-41.

22. Katzman WB, et al. Targeted spine strengthening exercise and posture training program to reduce hyperkyphosis in older adults: results from the study of hyperkyphosis, exercise, and function (SHEAF) randomized controlled trial. Osteoporos Int, 2017. 28(10): p. 2831-2841.

23. Kaijser Alin C, et al. Effect of treatment on back pain and back extensor strength with a spinal orthosis in older women with osteoporosis: a randomized controlled trial. Arch Osteoporos, 2019. 14(1): p. 5.

24. Ameis A, et al. The Global Spine Care Initiative: a review of reviews and recommendations for the non-invasive management of acute osteoporotic vertebral compression fracture pain in low- and middle-income communities. Eur Spine J, 2018. 27(Suppl 6): p. 861-869.

25. Pfeifer M, et al. Effects of two newly developed spinal orthoses on trunk muscle strength, posture, and quality-of-life in women with postmenopausal osteoporosis: a randomized trial. Am J Phys Med Rehabil, 2011. 90(10): p. 805-15.

26. Pfeifer M, B Begerow and HW Minne. Effects of a new spinal orthosis on posture, trunk strength, and quality of life in women with postmenopausal osteoporosis: a randomized trial. Am J Phys Med Rehabil, 2004. 83(3): p. 177-86.

27. Ball JM, et al. Spinal extension exercises prevent natural progression of kyphosis. Osteoporos Int, 2009. 20(3): p. 481-9.

28. Zhang J, et al. Risk factors for conservative treatment failure in acute osteoporotic vertebral compression fractures (OVCFs). Arch Osteoporos, 2019. 14(1): p. 24.

29. Venmans A, et al. Natural history of pain in patients with conservatively treated osteoporotic vertebral compression fractures: results from VERTOS II. AJNR Am J Neuroradiol, 2012. 33(3): p. 519-21.

30. Muratore M, et al. Osteoporotic vertebral fractures: predictive factors for conservative treatment failure. A systematic review. Eur Spine J, 2018. 27(10): p. 2565-2576.

31. Papanastassiou ID, et al. Comparing effects of kyphoplasty, vertebroplasty, and non-surgical management in a systematic review of randomized and non-randomized controlled studies. Eur Spine J, 2012. 21(9): p. 1826-43.

32. Firanescu CE, et al. Vertebroplasty versus sham procedure for painful acute osteoporotic vertebral compression fractures (VERTOS IV): randomised sham controlled clinical trial. BMJ, 2018. 361: p. k1551.

33. Lewis G. Viscoelastic properties of injectable bone cements for orthopaedic applications: state-of-the-art review. J Biomed Mater Res B Appl Biomater, 2011. 98(1): p. 171-91.

34. Peng Y, et al. Optimizing bone cement stiffness for vertebroplasty through biomechanical effects analysis based on patient-specific three-dimensional finite element modeling. Med Biol Eng Comput, 2018. 56(11): p. 2137-2150.

35. Trout AT, DF Kallmes and TJ Kaufmann. New fractures after vertebroplasty: adjacent fractures occur significantly sooner. AJNR Am J Neuroradiol, 2006. 27(1): p. 217-23.

36. Polikeit A, LP Nolte and SJ. Ferguson. The effect of cement augmentation on the load transfer in an osteoporotic functional spinal unit: finite-element analysis. Spine (Phila Pa 1976), 2003. 28(10): p. 991-6. 
37. Ailon T, et al. Progressive Spinal Kyphosis in the Aging Population. Neurosurgery, 2015. 77 Suppl 4: p. S164-72.

38. Ailon T, et al. Degenerative Spinal Deformity. Neurosurgery, 2015. 77 Suppl 4: p. S75-91.

39. Macagno AE and MF O'Brien. Thoracic and thoracolumbar kyphosis in adults. Spine (Phila Pa 1976), 2006. 31(19 Suppl): p. S161-70.

40. Goldstein CL, D.S Brodke and TJ Choma. Surgical Management of Spinal Conditions in the Elderly Osteoporotic Spine. Neurosurgery, 2015. 77 Suppl 4: p. S98-107.

41. DeWald CJ and T Stanley. Instrumentation-related complications of multilevel fusions for adult spinal deformity patients over age 65: surgical considerations and treatment options in patients with poor bone quality. Spine (Phila Pa 1976), 2006. 31(19 Suppl): p. S144-51.

42. Ailon T, et al. Surgical considerations for major deformity correction spine surgery. Best Pract Res Clin Anaesthesiol, 2016. 30(1): p. 3-11.

43. Radcliff KE, et al. Adjacent segment disease in the lumbar spine following different treatment interventions. Spine J, 2013. 13(10): p. 1339-49.

44. Kanayama M, et al. Motion-preserving surgery can prevent early breakdown of adjacent segments: Comparison of posterior dynamic stabilization with spinal fusion. J Spinal Disord Tech, 2009. 22(7): p. 463-7.

45. Mageswaran P, et al. Hybrid dynamic stabilization: a biomechanical assessment of adjacent and supraadjacent levels of the lumbar spine. J Neurosurg Spine, 2012. 17(3): p. 232-42.

46. Strube P, et al. Dynamic stabilization adjacent to single-level fusion: part I. Biomechanical effects on lumbar spinal motion. Eur Spine J, 2010. 19(12): p. 2171-80.

47. Putzier M, et al. Dynamic stabilization adjacent to single-level fusion: part II. No clinical benefit for asymptomatic, initially degenerated adjacent segments after 6 years follow-up. Eur Spine J, 2010. 19(12): p. 2181-9.

48. Greiner-Perth R, et al. Dynamic posterior stabilization for degenerative lumbar spine disease: a large consecutive case series with long-term follow-up by additional postal survey. Eur Spine J, 2016. 25(8): p. 2563-70.

49. Galbusera F, et al. Pedicle screw loosening: a clinically relevant complication? Eur Spine J, 2015. 24(5): p. 1005-16.

50. Chou PH, et al. Could the Topping-Off Technique Be the Preventive Strategy against Adjacent Segment Disease after Pedicle Screw-Based Fusion in Lumbar Degenerative Diseases? A Systematic Review. Biomed Res Int, 2017. 2017: p. 4385620.

51. Diebo BG, et al. Sagittal deformities of the spine: factors influencing the outcomes and complications. Eur Spine J, 2015. 24 Suppl 1: p. S3-15.

52. Schwab FJ, et al. Risk factors for major peri-operative complications in adult spinal deformity surgery: a multi-center review of 953 consecutive patients. Eur Spine J, 2012. 21(12): p. 2603-10.

53. Bess $\mathrm{S}$, et al. Pain and disability determine treatment modality for older patients with adult scoliosis, while deformity guides treatment for younger patients. Spine (Phila Pa 1976), 2009. 34(20): p. 2186-90.

54. Richner-Wunderlin S, et al. Factors associated with having an indication for surgery in adult spinal deformity: an international european multicentre study. Eur Spine J, 2019. 28(1): p. 127-137.

55. Chen PG, et al. Surgery for Degenerative Lumbar Scoliosis: The Development of Appropriateness Criteria. Spine (Phila Pa 1976), 2016. 41(10): p. 910-8. 
56. Schwab F, et al. Scoliosis Research Society-Schwab adult spinal deformity classification: a validation study. Spine (Phila Pa 1976), 2012. 37(12): p. 1077-82.

57. Yilgor C, et al. Global Alignment and Proportion (GAP) Score: Development and Validation of a New Method of Analyzing Spinopelvic Alignment to Predict Mechanical Complications After Adult Spinal Deformity Surgery. J Bone Joint Surg Am, 2017. 99(19): p. 1661-1672.

58. Yagi $\mathrm{M}$, et al. Characterization and surgical outcomes of proximal junctional failure in surgically treated patients with adult spinal deformity. Spine (Phila Pa 1976), 2014. 39(10): p. E607-14.

59. Yagi M, KB Akilah and O Boachie-Adjei. Incidence, risk factors and classification of proximal junctional kyphosis: surgical outcomes review of adult idiopathic scoliosis. Spine (Phila Pa 1976), 2011. 36(1): p. E60-8.

60. Kim HJ, et al. Patients with proximal junctional kyphosis requiring revision surgery have higher postoperative lumbar lordosis and larger sagittal balance corrections. Spine (Phila Pa 1976), 2014. 39(9): p. E576-80.

61. Parreira PCS, et al. An overview of clinical guidelines for the management of vertebral compression fracture: a systematic review. Spine J, 2017. 17(12): p. 1932-1938.

62. Diebo BG, et al. From Static Spinal Alignment to Dynamic Body Balance: Utilizing Motion Analysis in Spinal Deformity Surgery. JBJS Rev, 2018. 6(7): p. e3. 


\section{VALORIZATION}

Spinal disorders are common and have a substantial impact on both patients and society, affecting more than 1.7 billion people worldwide. With aging of our population the burden of spinal disorders on society, in terms of quality of life and costs, is expected to increase further. For instance, the risk of fractures after falling in people with osteoporosis is high due to increased bone fragility. These fragility fractures are associated with significant mortality, morbidity, and low quality of life. The economic burden of incident and prior fragility fractures is huge (approximately 37 billion euros in 2010 for Europe) and the costs are expected to increase by $25 \%$ in 2025 .

New osteoporotic vertebral compression fractures (OVCFs) occur in approximately 500,000 patients per year in Europe. In this thesis it was shown that after an OVCF patients do not recover to their previous level of mobility. This is of high clinical importance, since impairments in balance and gait are the primary cause of recurrent falls. Early diagnosis of balance disorders after an OVCF is warranted to prevent osteoporotic patients from falling and the development of novel fragility fractures. Moreover, it was shown that dynamic bracing may prevent or slowdown the decline in postural control and the subsequent progression of hyperkyphosis with resultant global sagittal malalignment and imbalance. Prevention of falls by means of gait training or dynamic bracing could thus lead to improved patient outcomes with less chance of developing new (fragility) fractures and a subsequent hyperkyphotic deformity. Currently we are working on a novel multicenter, randomized controlled trial for the conservative treatment of OVCFs.

For OVCFs that are not responsive to conservative treatment, percutaneous vertebroplasty can be considered. In this thesis a novel cement based on gold-containing polymethamethylacrylate (PMMA) microspheres was studied. While retaining mechanical characteristics, this novel cement may provoke less inflammatory reactions as compared to the commercially available bone cements. Adjacent level complications could potentially be prevented by adjustment of the mechanical properties of PMMA cement. As the stiffness of the cement can be modified, further study is needed to examine its optimal composition and potential utility in clinical practice.

The prevalence of adult spinal deformity is increasing rapidly due to aging, demographic shifts, increased life expectancy and an increased recognition of the disorder. Adult spinal deformity has a significant and measurable impact on health-related quality of life: The International Quality of Life Assessment Project surveyed almost 25,000 people from eight industrialized nations. Compared with other chronic age-related medical conditions (including arthritis, diabetes, pulmonary disease, and heart disease), patients with spinal deformity reported significantly worse scores for pain, functional status, mental health, and social functioning. Patients with sagittal imbalance reported especially severe disability. The spinal community is challenged to manage this increasing number of spinal disorders specifically related to the elderly. Both conservative and 
operative treatment can be complex in these often fragile patients because of physical deconditioning, medical comorbidities, balance and gait problems with subsequent risk of falling, and poor bone quality with concomitant risk of poor operative fixation and new fractures.

Adult spinal deformity surgery therefore is associated with high mechanical failure rates and proximal junctional failure (8-42\%), for which the mechanical stiffness mismatch between rigid spinal instrumentation and low bone mineral density because of osteoporosis, is an important determinant. In this thesis, a less rigid posterior based instrumentation system by means of polycarbonate-urethane rods, has been introduced which allows for a more physiological load distribution to the adjacent segments. This system could be used for topping-off as the semirigid zone provides a gradual transition from the rigid to mobile segments to lower the stress concentration at transitional levels. The aim of this method is to decrease the incidence of adjacent segment failure. Future studies are required to test the feasibility of polycarbonate-urethane rods for this specific indication.

In addition to the significant impact on health and functional status, adult spinal deformity results in a sizeable use of resources, of which surgery is the most important determinant of outcome, risk of complications and associated costs. Despite the importance and prevalence of adult spinal deformity, variability exists in the provision of surgical or nonsurgical treatment. This high variability in management is a reflection of the lack of an evidence-based approach to care. The appropriateness criteria were developed to guide clinical decision for specific subpopulations of patients, and to limit over- and underuse of surgery. Overuse occurs when patients undergo unnecessary procedures. Conversely, underuse occurs when patients are not offered appropriate care, such as if nonsurgical specialists do not appreciate that a patient with degenerative lumbar scoliosis and sagittal plane imbalance could achieve substantial improvements with surgery. To this end, the prevention of over- and underuse of surgery will have a substantial impact on both patients and society. In this thesis it was shown that the implementation of Patient Reported Outcome Measures into the appropriateness criteria for degenerative lumbar scoliosis surgery allows for an even more patient-centered, quantifiable, transparent and uniform clinical decision-making process.

When operative treatment is indicated, it is important to obtain the best result possible by tailoring operation to the individual patient. For this purpose, we have shown that the Global Alignment and Proportion (GAP) score provides an excellent patientspecific guide for operative correction of spinopelvic alignment, and we suggest that the score will be incorporated in clinical practice for operative planning to improve both the quality and cost effectiveness of care.

In conclusion, spinal aging will demand more and more complex and intensive treatment due to the confluence of an aging population and an increased capacity and willingness to manage difficult problems in older patients. In the current thesis both 
clinical and preclinical aspects of spinal aging were studied. In anticipation of aging of the population, one of the purposes of this thesis was to emphasize the significant and growing burden of spinal disorders in the elderly; to optimize current conservative and operative treatment for spinal aging; and to demonstrate that allocation of resources to the management of spinal disorders should be a priority for our healthcare economy. 



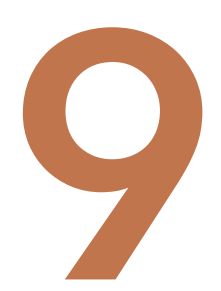

\title{
Summary
}

Nederlandse Samenvatting

\author{
List of Abbreviations
}





\section{SUMMARY}

For the coming decades, degenerative changes and deformity of the spine as a result of aging, will become a major challenge to the spinal community regarding evaluation and management. The global population is currently shifting upwards in age structure. As a result, clinicians worldwide will be required to manage an increasing number of spinal disorders specific to the elderly and aging of the spine. Spinal pathology in the elderly typically encompasses osteoporosis and osteoporotic vertebral compression fractures, and degenerative spinal deformity. The impact of these spinal disorders on health-related quality of life is more severe than the impact of many common diseases and impact more than 1.7 billion people worldwide. As a result of this substantial burden for both patients and society, the management of disorders in the aging spine should be a significant priority in our healthcare economy. Increased resources for research, innovation, and clinical care are warranted. In this thesis both clinical and preclinical aspects of spinal aging were studied.

The thesis consists of two major parts. The first part (Chapter 2-5) focuses on the osteoporotic vertebral compression fractures (OVCFs); The second part (Chapter 6 and 7) focuses on degenerative spinal deformity (or adult spinal deformity, ASD).

One factor related to disability in people with adult spinal deformity is decreased postural control and increased risk of falling. In Chapter 2, walking pattern and balance of postmenopausal women with and without OVCFs were compared. It was shown that patients suffering from an OVCF appeared to walk with significantly shorter and wider strides as compared to their healthy counterparts. Additionally, they adopt a less stable body configuration in the anterior direction, potentially increasing their risk of forward falls if perturbed. Although stride time and stride length improve over time after fracture, and reach healthy levels again, patients significantly deviate from normal gait patterns in stability and step width after six months of conservative treatment. Current conservative treatment of symptomatic OVCFs is multimodal and comprises analgesics, medication for osteoporosis, physical therapy and bracing. As patients do no fully recover to their previous level of mobility, it appears of high importance to add balance and gait training to the treatment algorithm of OVCFs.

Primarily, treatment of OVCFs should intend to relief pain, slow down the decline in postural control and limit the risk of falls and further fractures in this frail patient group. A second important goal in the treatment of OVCFs is the prevention of recurrent OVCFs and the subsequent progression of hyperkyphosis and resultant global sagittal malalignment. The role of bracing in this respect is controversial. In Chapter 3, the effect of a semirigid thoracolumbar orthosis on gait and balance in patients suffering from an 
OVCF was examined. At baseline, dynamic bracing caused a significantly more upright posture during walking and patients walked faster, with larger strides, longer stride times, and lower cadence compared to walking without brace. After six weeks of continues bracing, radiographic and dynamic sagittal alignment had improved compared to baseline. However, the observed effect disappeared after six months when the brace was not worn anymore, suggesting that the improvement in sagittal alignment is slightly reversible. Although preliminary, the results of this study could indicate that dynamic bracing using a semirigid, thoracolumbar spinal orthosis may be a useful addition to the multimodal treatment of OVCFs, in order to maintain congruent posture. However, since the effect seemed to be reversible, lifetime lumbodorsal muscle exercises should be provided, in order to prevent subsequent vertebral fractures. The study was limited by a relatively small number of patients; A large, prospective, randomized controlled trial will be needed to study the true effectiveness of dynamic bracing in terms of pain reduction and improved gait and balance in patients with an OVCF. However, initial clinical results are promising.

Only $60 \%$ of conservatively treated patients with acute OVCFs have sufficient pain relief and a good functional recovery within approximately three months after the fracture. However, $40 \%$ still has disabling pain after one year. For those patients who experience insufficient response to conservative therapy, interventional treatment by percutaneous vertebroplasty may be considered. In percutaneous vertebroplasty bone cement is carefully injected into the fractured vertebra under continuous fluoroscopic guidance. For radiopacity barium sulphate has been added to the polymethylmethacrylate (PMMA) cement, which has a number of drawbacks, including adverse effects on injectability, viscosity profile, setting time, mechanical properties of the cement and bone resorption. In Chapter 4, a novel PMMA cement, designed to address some of these drawbacks was presented. Its powder includes PMMA microspheres in which gold particles are embedded and its monomer ins the same as that used in commercial cements for vertebroplasty. The novel cement was compared to a commercial cement brand and it was demonstrated that the new cement has improved handling properties and is superior in terms of cytocompatibility. The compressive properties of the cement are similar to those of commercially available cement and the mechanical and filling properties were not compromised.

Early treatment of OVCFs is of high clinical importance in order to prevent subsequent vertebral fractures (otherwise known as the vertebral fracture cascade) and the subsequent increased thoracolumbar kyphosis and resultant global sagittal malalignment. Loss of sagittal alignment can cause severe pain, disability and progressive neurological deficits. When conservative treatment fails, spinal fusion using rigid instrumentation is currently the salvage treatment to stop further curve progression and further clinical de- 
cline. However, fusion surgery in this population is associated with high revision rates. The mechanical stiffness mismatch between the rigid spinal instrumentation and low bone mineral density in case of osteoporosis has been suggested to be a major contributor to these high failure rates. In Chapter 5, the effect of variation in rod stiffness on the loading of the anterior spinal column was studied, as measured by intradiscal pressure (IDP) of fixed spinal segments during flexion-compression. Low implant stiffness was found to generate lower IDP during flexion-compression loading, representing a more physiological loading pattern in comparison to high implant stiffness.

Surgery for adult spinal deformity (ASD) is a challenging and complex procedure. Chapter 6 and 7 elaborate on clinical decision-making in the treatment of ASD. The most common adult spinal deformity is degenerative lumbar scoliosis (DLS). Management of DLS is characterized by a substantial variety in treatment advice as there is no generally acknowledged consensus for decision-making. To increase uniformity and transparency of care as well as to improve evidence-based clinical decision-making, the Scoliosis Research Society (SRS) established the Appropriateness Criteria of Surgery for DLS. In these criteria, however, the patient perspective was not formally incorporated. In Chapter 6, the extent to which the patient perspective was integrated into the appropriateness criteria of surgery for DLS was evaluated. It was found that there was a significant association between the appropriateness of surgery and Patient Reported Outcome Measures (PROMs). Since pain and disability should determine the treatment modality in older patients, the implementation of PROMs into the appropriateness criteria may allow for a more transparent, quantifiable and uniform clinical decision-making process.

Despite the high reported complication and revision rates, for well-selected adult spinal deformity patients surgical intervention can offer superior clinical and radiographic outcomes compared to nonoperative treatment. An important cause of mechanical complications after ASD surgery is failure to achieve or maintain adequate postoperative sagittal alignment. The Scoliosis Research Society (SRS)-Schwab classification and the Global Alignment and Proportion (GAP) score were established to define appropriate surgical targets. In Chapter 7, the ability of the Schwab classification and the GAP score to predict mechanical complications following adult spinal deformity surgery was assessed and compared. The results demonstrated that both classification systems are capable of predicting radiographic evidence of mechanical failure, although the GAP score proved to be significantly better. 


\section{NEDERLANDSE SAMENVATTING}

"Spinal aging" betreft het ontstaan van degeneratieve veranderingen en deformiteiten van de wervelkolom. Spinal aging omvat een breed spectrum van aandoeningen waaronder osteoporose, osteoporotische wervelinzakkingsfracturen en degeneratieve wervelkolomdeformiteiten. Wereldwijd lijden momenteel meer dan 1.7 miljard patiënten aan spinal aging en dit aantal zal, ten gevolge van de vergrijzing, de komende decennia aanzienlijk toenemen. De negatieve impact van deze ziektebeelden op de kwaliteit van leven alsmede op de maatschappij is groot. Daarom dient de behandeling van spinal aging hoge prioriteit toegekend te krijgen in onze zorgeconomie. Nieuwe financiële bronnen dienen dan ook aangesproken te worden voor klinische zorg, onderzoek en innovatie. In dit proefschrift worden een aantal klinische en preklinische aspecten van spinal aging nader onderzocht.

Het proefschrift bestaat uit twee delen. In het eerste deel (Hoofdstuk 2-5) ligt de focus met name op de osteoporotische wervelinzakkingsfracturen. In het tweede deel (Hoofdstuk 6 en 7) ligt de nadruk op degeneratieve wervelkolomdeformiteiten (ook wel Adult Spinal Deformity (ASD) genoemd).

Patiënten met wervelkolomdeformiteiten zijn bekend met een veranderd looppatroon, een verminderde lichaamsbalans en een verhoogd valrisico. Over het looppatroon, de lichaamsbalans en het valrisico van patiënten met een osteoporotische wervelfractuur is slechts weinig informatie beschikbaar. In Hoofdstuk $\mathbf{2}$ worden het looppatroon en de lichaamsbalans van postmenopauzale vrouwen met én zonder een osteoporotische wervelinzakkingsfractuur vergeleken. Er wordt aangetoond dat patiënten met een inzakkingsfractuur een significant ander looppatroon kennen dan de gezonde populatie. Patiënten met een inzakkingsfractuur tonen een kortere staplengte en een grotere stapbreedte. Daarnaast is de stabiliteit in de voor-achterwaartse richting aanzienlijk verminderd, waarbij het valrisico potentieel is toegenomen wanneer patiënten zich verstappen of struikelen. Hoewel de staptijd en de staplengte gedurende het genezingsproces normaliseren, blijft het looppatroon ten aanzien van stabiliteit en stapbreedte significant anders ten opzichte van de gezonde populatie, zelfs zes maanden na conservatieve behandeling. De huidige conservatieve behandeling van symptomatische osteoporotische wervelinzakkingsfracturen is multimodaal en betreft analgetica, medicatie tegen osteoporose, fysiotherapie en bracing. Daar patiënten ten aanzien van mobiliteit niet volledig herstellen ondanks deze behandeling, is het van groot belang om loopscholing en stabiliteitstraining toe te voegen aan het behandelalgoritme van osteoporotische wervelinzakkingsfracturen. 
Het primaire doel van de behandeling van osteoporotische wervelinzakkingsfracturen is pijnverlichting en het optimaliseren van de lichaamsbalans om het valrisico en het risico op additionele fracturen bij deze kwetsbare patiëntengroep te verlagen. Een tweede belangrijk doel is preventie van nieuwe inzakkingsfracturen en de daaropvolgende progressie naar hyperkyfose en malalignement in het sagittale vlak. De rol van bracing hierin is controversieel. In Hoofdstuk $\mathbf{3}$ wordt het effect van een semirigide thoracolumbale brace (dynamic bracing) op het looppatroon en op de lichaamsbalans bij patiënten met een osteoporotische wervelinzakkingsfractuur onderzocht. Direct na het aanmeten van de brace werd een meer verbeterd looppatroon en een betere sagittale balans gedurende het mobiliseren geobjectiveerd dan tijdens het mobiliseren zonder brace. Nadat patiënten de brace zes weken continu gedragen hadden, was er sprake van een verbetering van zowel de radiografische als de dynamische sagittale balans ten opzichte van de eerste meting. Echter, het geobserveerde effect verdween na zes maanden wanneer de brace niet meer continu gedragen werd. Deze resultaten impliceren dat de verbetering in het sagittale vlak mogelijk reversibel is. Hoewel de resultaten nog preliminair zijn, lijkt de toevoeging van een semirigide thoracolumbale brace mogelijk wel een waardevolle bijdrage in het multimodale behandelalgoritme van wervelinzakkingsfracturen om een congruent postuur te waarborgen. Echter, daar het effect reversibel lijkt, is het van groot belang om ook levenslang core stability training te adviseren. De belangrijkste limitatie van deze studie is het kleine aantal patiënten. Een grote, prospectieve, randomized controlled trial is geïndiceerd om beter inzicht te krijgen in het ware effect van dynamic bracing in de behandeling van osteoporotische wervelfracturen.

Slechts $60 \%$ van de conservatief behandelde patiënten met symptomatische osteoporotische wervelinzakkingsfracturen reageert adequaat op conservatieve behandeling binnen drie maanden. Echter, na één jaar conservatieve behandeling blijft circa $40 \%$ invaliderende pijnklachten houden. Voor deze patiënten dient percutane vertebroplastiek overwogen te worden. Bij deze procedure wordt onder röntgendoorlichting botcement geïnjecteerd in de gefractureerde wervel. Ten behoeve van de radiopaciteit wordt bariumsulfaat toegevoegd aan het polymethylmethacrylate (PMMA) cement. Deze toevoeging kent enkele (negatieve) bijwerkingen ten aanzien van injecteerbaarheid, viscositeitsprofiel, setting time, mechanische eigenschappen en botresorptie. In Hoofdstuk 4 wordt een nieuw PMMA cement gepresenteerd, het poeder omvat PMMA microsferen met geïncorporeerde goud partikels, het vloeibare monomeer is hetzelfde als het monomeer dat gebruikt wordt in reguliere botcementen. Het nieuwe cement wordt vergeleken met een commercieel verkrijgbaar cement en er wordt aangetoond dat het nieuwe cement verbeterd is in "handling properties" en cytocompatibiliteit. Ook zijn de mechanische en de "filling" eigenschappen van het cement vergelijkbaar met die van het reguliere botcement. 
Tijdige, adequate behandeling van osteoporotische wervelinzakkingsfracturen voorkomt nieuwe inzakkingsfracturen en is derhalve van groot klinisch belang. Daarnaast voorkomt vroege behandeling ook gedeeltelijk de progressie naar thoracolumbale hyperkyfose en sagittaal malalignement. Sagittaal malalignement kan leiden tot ernstige pijnklachten, verminderde mobiliteit en progressieve neurologische beperkingen. Wanneer conservatieve behandeling onvoldoende verlichting biedt voor deze patiënten, is operatieve correctie middels lang-segment spondylodese (fusie) geïndiceerd om klinische achteruitgang te voorkomen. Echter, fusie-chirurgie bij deze kwetsbare patiëntenpopulatie kent hoge revisiecijfers. De zogenaamde "mismatch" in mechanische stijfheid tussen de rigide implantaten en de lage botdichtheid ten gevolge van osteoporose is een van de grootste oorzaken hiervan. In Hoofdstuk 5 wordt het effect van verschillende stijfheden van implantaten onderzocht op de druk in de tussenwervelschijf tijdens een flexie-compressie beweging van de wervelkolom. In deze studie wordt aangetoond dat een lagere stijfheid zorgt voor een lagere druk in de tussenwervelschijf. Deze lagere druk kent een meer fysiologisch patroon in vergelijking met de druk gemeten bij implantaten met een hogere stijfheid.

De operatieve correctie van wervelkolomdeformiteiten (Adult Spinal Deformity, ASD) is uitdagend en complex. In Hoofdstuk 6 en $\mathbf{7}$ ligt de nadruk op de klinische besluitvorming voor de behandeling van ASD.

De meest voorkomende vorm van ASD is degeneratieve lumbale scoliose (DLS). De behandeling van DLS wordt gekenmerkt door een substantiële variatie in behandelwijzen tussen verschillende specialisten aangezien er geen eenduidige consensus bestaat. Ten behoeve van een meer uniform, transparant en evidence-based behandelalgoritme heeft de Scoliosis Reserach Society (SRS) de zogenaamde "Appropriateness Criteria of Surgery for DLS” geïntroduceerd. In deze criteria wordt echter het perspectief van de patiënt zelf onvoldoende meegenomen. In Hoofdstuk 6 wordt onderzocht in welke mate het patiënten-perspectief (in de vorm van Patient Reported Outcome Measures (PROMs)) al dan niet geïntegreerd is in de "Appropriateness Criteria of Surgery for DLS". De uitkomst van deze studie toont aan dat er een significante associatie bestaat tussen de criteria en de PROMs. Maar aangezien pijn en de ervaren beperkingen van de patiënt leidend zouden moeten zijn in de besluitvorming, kan de implementatie van PROMs aan de "Appropriateness Criteria” zorgen voor een meer transparant, kwantificeerbaar en uniform besluitvormingsproces.

Ondanks de hoge complicatie- en revisiecijfers na operatieve behandeling van ASD, leidt operatieve correctie wel tot superieure klinische en radiografische resultaten in vergelijking met de conservatieve behandeling. Een (andere) belangrijke oorzaak van de mechanische complicaties na operatieve correctie is het feit dat er vaak sprake is van malalignement in het sagittale vlak. De "Scoliosis Research Society (SRS)-Schwab 
classificatie" en de "Global Alignment and Proportion (GAP) score" werden ontwikkeld om juiste chirurgische doelstellingen te definiëren ter preventie van sagittaal malalignement. In Hoofdstuk 7 worden deze twee predictiemodellen met elkaar vergeleken. In deze studie wordt aangetoond dat beide classificatiesystemen in staat zijn de mechanische complicaties na ASD chirurgie te voorspellen; echter, de GAP score bleek hiertoe significant beter. 


\section{LIST OF ABBREVIATIONS}

ASD

AUC

$\mathrm{BaSO}_{4}$

BMD

BoS

CAREN

CCI

CoM

CV

DALYs

DLS

GAP

GSA

GT

HADS

HR-pQCT

HRQOL

IDP

IRB

LDI

LL

MoS

ODI

OVCF

PCS

PCU

PEEK

PI

PJF

PJK

PMMA

PPS

PROMs

PT

QUALEFFO

RLL

ROC
Adult Spinal Deformity

Area Under the Curve

Barium sulphate

Bone Mineral Density

Base of Support

Computer Assisted Rehabilitation Environment

Charlson Comorbidity Index

Centre of Mass

Coefficient of Variation

Disability Adjusted Life Years

Degenerative Lumbar Scoliosis

Global Alignment and Proportion score

Global Sagittal Alignment

Global Tilt

Hospital Anxiety and Depression Scale

High Resolution peripheral Quantitative Computed Tomography

Health Related Quality of Life

Intradiscal pressure

Institutional Review Board

Lordosis Distribution Index

Lumbar Lordosis

Margins of Stability

Oswestry Disability Index

Osteoporotic Vertebral Compression Fracture

Pain Catastrophizing Scale

Polycarbonate-Urethane

Polyetheretherketone

Pelvic Incidence

Proximal Junctional Failure

Proximal Junctional Kyphosis

Polymethylmethacrylate

Purchasing Power Standards

Patient Reported Outcome Measures

Pelvic Tilt

Quality of Life Questionnaire of the European Foundation for Osteoporosis

Relative Lumbar Lordosis

Receiver Operating Characteristic 
RPV

RSA

SEM

SF-36

SRS

SS

SVA

TK

TPA

T1-SPi

VAS

WHO

$\mathbf{X}_{\mathrm{CoM}}$
Relative Pelvic Version

Relative Spinopelvic Alignment

Scanning Electron Microscopy

Short Form 36

Scoliosis Research Society

Sacral Slope

Sagittal Vertical Axis

Thoracic Kyphosis

T1 Pelvic Angle

T1 Spinopelvic Inclination

Visual Analogue Scale

World Health Organization

Extrapolated Centre of Mass 



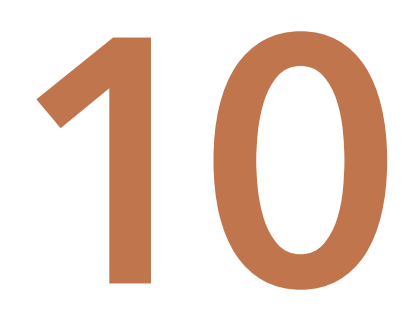

\title{
Dankwoord
}

\author{
Curriculum Vitae
}

List of Publications

List of Presentations 



\section{DANKWOORD}

Het dankwoord, het enige gedeelte in het proefschrift dat ik volledig zelfstandig geschreven heb. Onderzoek is namelijk teamsport, en behoudens dit dankwoord is alles in dit proefschrift tot stand gekomen door fijne samenwerkingen, goede supervisie en niet aflatende steun van verschillende personen. Ik wil dan ook iedereen die heeft bijgedragen aan het tot stand komen van dit werk hartelijk bedanken, en onderstaande personen in het bijzonder.

Mijn eerste persoonlijke woord van dank gaat uit naar mijn promotor prof. dr. L.W. van Rhijn. Beste Lodewijk, ik wil je bedanken voor het vertrouwen dat je in mij hebt en alle kansen die je me hebt aangeboden. Jij bent de afgelopen jaren een luisterend oor voor mij geweest, zowel voor werk- als privézaken. Jouw open houding, nuchterheid en aanstekelijke enthousiasme zijn voor mij een voorbeeld.

Mijn copromotor dr. P.C. Willems is de drijvende kracht geweest achter al het onderzoek gepresenteerd in dit proefschrift. Beste Paul, ik waardeer jouw altijd positieve instelling en drive. Nooit is iets teveel gevraagd en altijd vind je wel een momentje om even te sparren. Jouw expertise als wervelkolomchirurg was onmisbaar voor de totstandkoming van dit proefschrift.

Mijn andere copromotor dr. J.J. Arts, beste Chris, ook jou wil ik bedanken voor al je hulp. Ik waardeer je altijd positieve instelling en het feit dat je vanaf het eerste moment in 2008 achter me hebt gestaan!

De leden van de beoordelingscommissie prof. dr. R.A. de Bie, prof. dr. M. de Kleuver, prof. dr. F.C. Öner, Dr. J. Sieben en prof. dr. R.J.E.M. Smeets, veel dank voor uw tijdsinvestering in het kritisch lezen van en beoordelen van mijn proefschrift, alsmede voor de bereidheid zitting te nemen in de beoordelingscommissie. Dr. W. van Hemert, beste Wouter ik vind het bijzonder dat je wilt opponeren en het is een feest om bij jou en Inez op OK mee te mogen opereren!

Niet op de laatste plaats wil ik alle patiënten bedanken die deelnamen aan de onderzoeken gepresenteerd in dit proefschrift.

Beste co-auteurs, dank voor alle onmisbare hulp tijdens het schrijven van dit proefschrift. Beste prof. dr. van Royen en dr. Stadhouder, beste Barend en Agnita, dank voor de fijne samenwerking, hopelijk kunnen we dit voortzetten in de toekomst! Beste prof. dr. Koole, dr. Saralidze en dr. Brans, ook jullie mogen niet ontbreken. Dank voor de zeer prettige samenwerking en het mooie eindresultaat! Beste dr. Lataster, beste Arno, dank 
voor alle leerzame momenten op het anatomielab en alle geboden mogelijkheden. Ook aan Leon en Johan, bedankt, jullie zijn onmisbaar! Beste dr. van Kuijk, beste Sander, wat een verrijking om met jou samen te mogen werken. Je verstaat je vak fantastisch. Beste CAREN team (dr. Kenneth Meijer, Paul en Rik ) dank voor de alle hulp, dat er nog maar veel mooie studies mogen volgen in de toekomst. Beste Rachel, wat hebben we het vaak over dit moment gehad. Het was heerlijk om met je te werken en tegen je te klagen als het even tegen zat. Dear Chris, I am grateful for all your help and I hope to collaborate with you a lot in the future! Beste prof.dr. van den Bergh en dr. Bours ook jullie bedankt voor de fijne samenwerking tussen onze afdelingen. Joost, bedankt voor de fijne samenwerking en goede gesprekken. Sayf, ook jij mag niet ontbreken. Dank voor al je hulp, ik kijk uit naar onze samenwerking in de toekomst!

Beste stafleden orthopedie en traumachirurgie van het MUMC+, dank voor de orthopedische basis die jullie mij hebben gegeven en het aanstekelijk enthousiasme voor de orthopedie dat jullie samen hebben gegenereerd en hebben overgebracht. Beste Lodewijk, Paul, Heleen, René, Pieter, Mark, Peter, Jan, Joris, Adhiambo, Tim en Loek, mijn eerste voetstappen bij de orthopedie heb ik aan jullie te danken. Hier ben ik nog iedere dag blij mee. Ik heb van jullie allen veel geleerd als dokter, chirurg en als mens. De kwaliteit van de opleiding is hoog en de mogelijkheden oneindig. Ik kijk er naar uit om mijn laatste deel van de opleiding bij jullie af te ronden. Ook alle andere collega's van de operatiekamer, gipskamer, verpleegafdeling, SEH en poli wil ik hartelijk bedanken voor de interesse en fijne samenwerking. Karin, Gyorgy, Patrick, Monique en Anniek met jullie heb ik een bijzondere band over gehouden die mij veel waard is. Mariëlle, Gwen en Anne, dank voor de gezellige sportmomenten! Lieve Ramona en Alberto, bedankt voor de fijne vriendschap, dat er nog maar veel leuke momenten met z'n vieren mogen volgen.

Beste stafleden en ex-collega's van het Zuyderland MC: dank voor een fantastische ANIOS tijd bij jullie, jullie hebben mijn enthousiasme voor de orthopedie nog meer aangesterkt. Ook wil ik jullie nogmaals bedanken voor de steun in de tijd dat ik het even extra nodig had. Edwin hierin speciaal woord naar jou en Nicole: duizend maal dank voor alles! Ook Hans, Anja en Aart ontzettend bedankt voor de eeuwige interesse en het immer bestaande contact. Lieve Monique en Mark, en Rian jullie bedankt voor de bijzondere vriendschap.

Beste stafleden van de maatschap orthopedie en chirurgie van het Viecuri Medisch Centrum, dank voor de fantastische vooropleiding die ik bij jullie heb mogen volgen. Fijn dat jullie allen op eigen manier investeerden in mijn ontwikkeling als persoon en als arts. Renee, fijn dat we altijd contact gehouden hebben, hopelijk lukt het afspreken nu écht een keer. 
Beste Jan, Rob, Henk, Hans, Arnold, Marijn, Willem, Florens, Inge, Anouk, Coen, Richard en Anne, wat ben ik blij en dankbaar dat ik een gedeelte van de opleiding bij jullie in het Maxima mag doen. De topografie zou geen enkele AIOS ervan moeten weerhouden om hier een deel van de opleiding te volgen, het is een voorrecht! Paul en Niek, dank voor de fantastische dagen in het CZE! Beste collega arts-assistenten, beste Jaap, Joep, Frank J., Frank van R., Jetse, Freek, Vincent, Martijn, Bart, Merel, Wesley en mede spice girl Joost: dank voor de gezellige periode in het Maxima! Ook de collega's van de poli, gipskamer, de onderzoeksgroep en de operatiekamer mogen zeker niet vergeten worden want jullie spelen een belangrijke rol in het fijne opleidingsklimaat in het Maxima! Annie, dank dat je me in contact hebt gebracht met Ria. Ria dank voor het nakijken van de Nederlandse samenvatting.

Beste collega arts-assistenten van ROGO Zuid, dank voor de mooie opleidingstijd die we samen hebben. Aniek toch een speciaal woord voor jou. Dank voor onze bijzondere vriendschap. Je bent een geweldige dokter en je wordt een fantastisch orthopeed! Vincent jou wil ik ook in het bijzonder bedanken, fijn om zo'n groot deel van de opleiding met je samen te doen! Je bent een topper!

Collega's van het Laboratory of Experimental Orthopedics: Dr. T. Welting, Beste Tim, wat heb ik veel van jou geleerd. Als onderzoeker en als mens. Ik heb ontzag voor je passie voor het vak en je gedrevenheid. Don en Andy, de vaste krachten van het lab, jullie zijn onmisbaar. Gedurende de periode dat ik daar werkzaam was heb ik toch heel wat met jullie af gelachen en kon ik regelmatig m'n ei bij jullie kwijt waarvoor dank. Andy deze kun je er weer bij schrijven in je logboek: someday, somehow... Marloes, bijzonder dat we tegelijkertijd zijn begonnen en tegelijkertijd ook ons proefschrift mogen afronden! Je bent een kanjer en hoop dat we straks meer tijd vinden om gezellig samen te eten! Alex, je hebt een speciaal plekje in dit dankwoord meer dan verdiend. Met name gedurende de eerste fase van mijn proefschrift heb je me intensief geholpen. Je bent een briljant onderzoeker en ik heb veel van je geleerd. Ralph en Annemarijn, ik waardeer de vriendschap die we samen hebben opgebouwd en de gezellige etentjes. Dank ook voor de hulp bij de afronding van dit proefschrift. Ralph je bent een fantastische collega en ik kijk er naar uit om in de toekomst met je samen te werken! Maarten, ik volg al jaren een beetje jouw voetsporen. Fijn dat we zo'n goede band hebben opgebouwd! Ufuk, dank voor de mooie momenten op het lab, ik kijk uit naar de samenwerking in de kliniek. Jim, Marjolein, Guus, Nina, Mandy, Ellen: ook jullie bedankt voor een fantastische tijd op het lab.

Lieve Liesbeth, Margareth en Anita, al vanaf 2008 kom ik zo nu en dan mijn hart luchten op het trialbureau orthopedie. Het is heerlijk om dat bij jullie te mogen doen. Dank voor jullie oprechte interesse, gouden tips en afleiding. Lieve Mieke, jij bent de 
drijvende kracht geweest achter verschillende studies. Ondanks dat je het al super druk hebt deed je dit met veel plezier en liefde voor me. Dat etentje komt er echt want dat heb je meer dan verdiend! Dames van het secretariaat orthopedie: Manon, Marion, Jerney, Chantalle, Denise, Astrid, Wil, Helma en Marieke. Ook jullie bedankt voor alle oprechte interesse en hulp de afgelopen jaren. Denise een speciaal woord van dank aan jou, hopelijk zijn ze niet allemaal zo als ik ;-).

Lieve Karin en Thijs, Rob en Karlijn, Els en Frans, Pim en Anke, vijf jaar alweer na die zwarte dag op 17 juli 2014. De dag die zorgde voor een niet opvulbare leegte in onze levens: het verlies van onze lieve Kristy en Sjors. Wat mis ik ze enorm. Er gaat geen dag voorbij dat ik er niet aan denk. Tegelijkertijd heb ik veel bewondering voor jullie en ben ik dankbaar voor de bijzondere band die ik met jullie heb. Lieve Rhein ik koester ook de fijne band die ik met jou heb opgebouwd, dit betekent veel voor me.

Chantal en Ruby, en Lilian, eeuwig dankbaar ben ik jullie voor jullie eerste geslaagde koppelproject. Zonder jullie had ik Sjoke nooit leren kennen. Hierdoor heb ik ook Rian en Dave, Jody en Ricardo, en Marloes en Donné ontmoet. Bedankt voor de fijne vriendschap.

Lieve Darshini en Iris, we zien elkaar te weinig maar mede door jullie heb ik een fantastische tijd in Maastricht gehad waarvoor veel dank! Lieve Wes, fijn dat we sinds dit jaar de banden hebben aangehaald, je bent een goede vriend. Lieve Sandra en Caren, ik ben jullie dankbaar voor de vriendschap en de gezellige etentjes/uitjes de afgelopen jaren. Ik waardeer jullie om jullie eerlijkheid en oprechtheid en ik hoop dat we deze vriendschap nog lang kunnen voortzetten! Lieve Esther en Sophia, mijn studievriendinnetjes, zo fijn dat we nog een goed contact hebben gehouden na de studie. Nu de promotie erop zit kom ik jullie snel opzoeken! Lieve Madelon, ik vergeet nooit meer onze tijd als huisgenoten op de Koning Clovisstraat. Je bent een bijzondere vriendin en ik heb bewondering voor je ambities. Je bent goud waard. Lieve Sofie, Noortje, Sanne, Marloes en Stefanie. Wat speciaal dat we nog steeds zo'n bijzondere vriendschap hebben ondanks dat we verspreid zijn door heel Nederland. Ik ben jullie dankbaar hiervoor. Lieve Sofie, onze vriendschap bestaat inmiddels al meer dan 25 jaar. We zien elkaar veel te weinig maar wanneer we elkaar zien zijn de banden ook in no time weer aangehaald. Ik hoop nu de drukte wat minder wordt dat we elkaar weer wat meer gaan zien want je bent een topper! Lieve Michelle, een vriendin uit duizenden. Vanuit de handbalwereld is onze vriendschap ontstaan en die wordt ieder jaar weer sterker. Bedankt voor je luisterend oor, steun en aanmoedigende woorden, dit heeft mij opnieuw doen beseffen hoe belangrijk je voor me bent. Ik kijk er naar uit om nog vele leuke momenten te mogen beleven met jou en Mathijs. Lieve Mick, inmiddels al meer dan tien jaar geleden leerden wij elkaar kennen tijdens onze eerste onderwijsgroep. Ondanks dat onze wegen 
op het gebied van studie al vrij snel uit elkaar liepen, hebben wij er toch een hechte vriendschap aan over gehouden. En wat hebben we het vaak over deze dag gehad... Inmiddels woon je alweer een paar jaar in Madrid met je liefde Tania en toch zien we elkaar nog met enige regelmaat. Ik ben dankbaar voor je onvoorwaardelijke vriendschap en ben erg trots dat jij vandaag achter mij wil staan als paranimf. Tania thank you so much for your help designing the cover of this thesis, and thank you for being an amazing girlfriend for Mick.

Ik heb het voorrecht gehad om op te mogen groeien in twee geweldige families: familie de Graef en familie Jacobs. Wat ben ik hier trots op. Dank voor jullie oprechte interesse in mij en mijn onderzoek. Ook mijn schoonfamilies, familie Merk en familie Theunissen, wil ik bedanken voor het warme ontvangst in jullie families.

Lieve Rob en Karin, allereerst bedankt voor het tot stand komen en opvoeden van mijn fantastische en lieve Sjoke. Tevens kan ik jullie niet genoeg bedanken voor alles wat jullie iedere week weer voor Sjoke en mij doen. Jullie zijn geweldige schoonouders!

Liefste Ans en Leo, jullie horen bij uitstek thuis in dit dankwoord. Al heel mijn leven staan jullie voor me klaar. Mijn tweede ouders, zo voelt het echt. Het is een ware rijkdom om jullie in mijn leven te hebben.

Lieve Luna, mijn kleine zusje. Nou, dat ben je allang niet meer. Je bent gegroeid tot de prachtige vrouw die je nu bent. Je bent een kanjer in alle opzichten, ik gun je dat je jezelf eens door mijn ogen zou zien. Ik ben trots op jou en op de band tussen ons die steeds sterker wordt. Ook ben ik blij met jouw lieve Dirk. Dirk, je bent al zo lang bij ons dat ik me geen leven meer zonder je kan en wil voorstellen. Je bent goed voor Luna en daar ben ik je super dankbaar voor. Ik kijk er naar uit om al jouw mooie projecten te komen bewonderen de komende jaren!

Lieve Freek, wat ben ik trots op een broer als jij. Nog trotser dat je achter me wil staan als paranimf. Ik heb bewondering voor hoe je je dromen najaagt en dit alles combineert met je prachtige gezin. De drijvende kracht hierachter is dan ook niet zo maar iemand: Karin, je bent de beste schoonzus die ik me ooit heb kunnen wensen! Het klikte eigenlijk vanaf moment één dat we elkaar ontmoet hebben en die band is alleen maar sterker geworden. Je bent een topper! Uit de combinatie van jullie twee kan dan ook maar alleen goud voortkomen, en zo is ook gebleken: mijn allerliefste nichtjes Mila en Floor. Lieve meiden, jullie hebben geen idee hoeveel jullie mijn leven de afgelopen jaren verrijkt hebben. Het is een feestje jullie tante te mogen zijn. 
Liefste pap en mam, woorden schieten te kort. Ik ben jullie dankbaar voor alles. Dankbaar voor jullie niet aflatende steun in het nastreven van onze doelen en jullie onvoorwaardelijke liefde. Zonder jullie was ik niet wie ik nu ben, en was ik niet waar ik nu ben. Mam, je immer positieve en optimistische instelling zijn een waar voorbeeld voor me. Pap, wat ben je een harde werker en een doorzetter en wat lijken we toch vreselijk veel op elkaar. Beiden hebben jullie mij belangrijke eigenschappen meegegeven die essentieel waren bij het schrijven en afronden van dit proefschrift. Ik had me geen betere ouders kunnen wensen.

Tot slot, allerliefste Sjoke, wij leerden elkaar kennen toen ik al volop bezig was met het verwezenlijken van al mijn dromen. Je stapte in op een trein waarvan je achteraf vast nog vaak hebt gedacht: 'Waar ben ik aan begonnen...'. Desalniettemin ben je mijn onvoorwaardelijke steun en toeverlaat en daar kan ik je niet genoeg voor bedanken. Wat hebben we het fijn samen. Ik hoop dat dat voor altijd zo mag blijven. 



\section{CURRICULUM VITAE}

Eva Jacobs was born in Weert, the Netherlands on May 22 , 1989. In 2007, she graduated from highschool (Gymnasium, Philips van Horne, Weert) and started medical school at the Maastricht University in the Netherlands. In 2008 she started her research career at the Orthopaedic Research Laboratory Maastricht under supervision of prof. dr. Lodewijk W. van Rhijn and dr. Paul C. Willems. During her studies she worked as coordinator and surgery planner for the "Sport en Bewegen" programme,

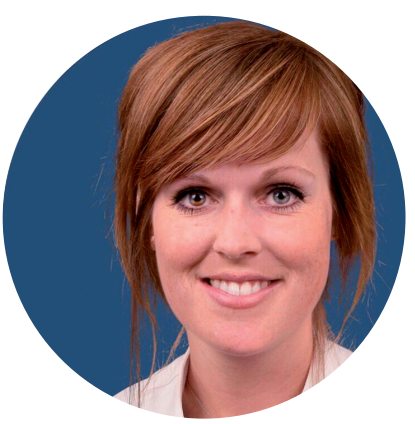
Department of Orthopaedic Surgery, Maastricht University Medical Center (MUMC+). In August 2013 Eva completed her medical degree and started working as medical trainee at the Department of Orthopaedic Surgery, Orbis Medical Center Sittard, the Netherlands (under supervision of dr. N.P. Kort). From February 2015 until March 2016 she worked as a PhD candidate at the Orthopaedic Research Laboratory Maastricht.

In 2016 she started her general surgical training at the Viecuri Medical Center, Venlo, the Netherlands (under supervision of dr. H.M.J. Janzing) as part of her specialist training in orthopaedic surgery. She continued her orthopaedic surgery training at the MUMC+ (under supervision of dr. H.M. Staal) and currently prolonged her residency at the Maxima Medical Center, Veldhoven (under supervision of dr. R.P.A. Janssen), the Netherlands.

During her residency she continued her research under supervision of prof. dr. Lodewijk W. van Rhijn, dr. Paul C. Willems and dr. Chris J. Arts, MUMC+. Furthermore, she is enrolled in the Eurospine Diploma programme. She will complete her orthopaedic training in 2022 and obtain the Eurospine Basic Diploma in November 2019. 


\section{LIST OF PUBLICATIONS}

- Jacobs E, Groot D, Das M, Hermus JP. Pseudoaneurysm of the anterior tibial artery after ankle arthroscopy.

J Foot Ankl Surg 2011; 50(3): 361-3

- Jacobs E, Feczko P, Emans E. Recurrent patella loosening and extra-articular migration after TKA.

J Knee Surg 2013; 26: S100-2

- Roth AK, Bogie R, Jacobs E, Arts JJ, van Rhijn LW. Large animal models in fusionless scoliosis correction research: a literature review.

Spine J 2013; 13(6):675-88

- Jacobs E, Witlox MA, Hermus JP. Fribroma of tendon sheath located within Kager's triangle.

J Foot Ankl Surg 2014; 53(2):208-11

- Jacobs E, Saralidze K, Roth AK, Jong JJA, van den Bergh JPW, Knetsch MLW, Brans B, Lataster A, Dkordevic I, Willems PCPH, Koole LH. Synthesis and characterization of a new vertebroplasty cement based on gold-containing PMMA microspheres.

Biomaterials 2016; 82: 60-70

- Jacobs E, Roth AK, Arts JJ, van Rhijn LW, Willems PC. Reduction of intradiscal pressure by the use of polycarbonate-urethane rods as compared to titanium rods in posterior thoracolumbar spinal fixation.

J Mater Sci Mater Med. 2017 Aug 21;28(10):148

- Jacobs E, van Kuijk SMJ, Merk JMR, Vandewall-Peeters M, Jütten-Brouwer LMC, van Rhijn LW, Willems PC. The implementation of patient reported outcome measures in appropriateness criteria of surgery for degenerative lumbar scoliosis.

Spine J. 2019 Apr;19(4):655-661

- Jacobs E, van Kuijk SMJ, Stadhouder A, van Royen B, van Rhijn LW, Willems PC. Prediction of mechanical complications in adult spinal deformity surgery - The GAP score versus the Schwab classification.

Spine J. 2019 May;19(5):781-788 
- Jacobs E, van Kuijk SMJ, Merk JMR, Vandewall-Peeters M, Jütten-Brouwer LMC, van Rhijn LW, Willems PC. Response letter to the editor entitled 'Prediction of mechanical complications in adult spinal deformity surgery: methodological issues.'

Spine J. 2019 May;19(5):960

- Jacobs E, Senden R, McCrum C, van Rhijn LW, Meijer K, Willems PC. The effect of a semirigid thoracolumbar orthosis on gait and sagittal alignment in patients with an osteoporotic vertebral compression fracture.

Clin Interv Aging. 2019 Apr 11;14:671-680

- Jacobs E, Senden R, McCrum C, van Rhijn LW, Meijer K, Willems PC. Gait in patients with symptomatic osteoporotic vertebral compression fractures over six months recovery.

Aging Clin Exp Res. 2019 Apr 27 


\section{LIST OF PRESENTATIONS}

- Jacobs E, Roth AK, Willems PCPH, Arts JJ, van Rhijn LW. Thoracic hyperkyphosis correction in osteoporotic patients: Polycarbonate-Urethane Spine Implants'.

Presentation annual meeting Nederlandse Orthopedie Vereniging (NOV), Rotterdam, the Netherlands (06-02-2014)

- Jacobs E, Roth AK, Willems PCPH, Arts JJ, van Rhijn LW. Thoracic hyperkyphosis correction in osteoporotic patients: Polycarbonate-Urethane Spine Implants'.

Poster presentation Orthopedic Research Society (ORS), Annual Meeting, New Orleans, USA (0603-2014)

- Jacobs E, Roth AK, Willems PCPH, Arts JJ, van Rhijn LW. Thoracic hyperkyphosis correction in osteoporotic patients: Polycarbonate-Urethane Spine Implants'.

Poster presentation annual meeting Biomedica, Maastricht, the Netherlands (17-06-2014)

- Koole LH, Saralidze K, Jacobs E, Roth AK, Willems PC. Gold-containing PMMA microspheres. A route to new highly radiopaque cements for vertebroplasty.

European Society for Biomaterials, Liverpool, United Kingdom (31-08-2014)

- Jacobs E, van Kuijk SMJ, Merk JMR, Jütten-Brouwer L, Vandewall-Peeters M, van Rhijn LW, Willems PC. The implementation of health-related quality of life instruments in appropriateness criteria of surgery for degenerative lumbar scoliosis.

Podium presentation annual meeting North American Spine Society (NASS) 2018. Nominated for the Value Care Award and the Resident Award 2018 

